\title{
Unmoderated Posters
}

\begin{abstract}
UP-001
Orthotopic Continent Urinary Diversion vs. Ileal Conduit: Comparison of Intraoperative and In-hospital Complications, Blood Transfusion, Length of Stay, and In-hospital Mortality

Djahangirian, Orchidee; Schmitges, Jan; Tian, Zhe; Karakiewicz, Pierre; Ismail, Salima

Cancer Prognostics and Health Outcomes Unit, University of Montreal Health Centre, Montreal, QC, Canada

Introduction and Objectives: Orthotopic continent urinary diversion (OCUD) after radical cystectomy (RC) may predispose to a higher rate of complications than ileal conduit urinary diversion (ICUD).

Methods: We identified all OCUD and ICUD patients in the Nationwide Inpatient Sample (NIS). We tabulated intraoperative and in-hospital complications, transfusion, length of stay, and in-hospital mortality rates according to urinary diversion. Multivariable analyses were performed, with adjustment for age, Charlson comorbidity Index $(\mathrm{CCl})$, gender, race, hospital type and region, annual hospital volume tertiles, and year of surgery.

Results: The rate of OCUD was $8 \%$ vs. $92 \%$ for ICUD. Intraoperative and in-hospital complication rates were $2.5 \%$ vs. $2.6 \%(p=0.8)$ and $24.7 \%$ vs. $28.1 \%(p=0.04)$ for OCUD and ICUD patients, respectively. Blood transfusions were administered in $17.9 \%$ vs. $26.7 \%(p<0.001)$. Length of stay above median ( $\geq 9$ days) was recorded in $53.5 \%$ vs. $54.9 \%(p=0.4)$. In-hospital mortality was $0.4 \%$ vs. $2.5 \%(p<0.001)$. After adjusting for all covariates, OCUD patients were less likely to receive a blood transfusion (odds ratio [OR]: $0.7, p<0.001$ ), more likely to have an increased length of stay (OR: $1.3, p=0.002$ ), and less likely to succumb to in-hospital mortality (OR: $0.3, p=0.03$ ) relative to ICUD patients. Urinary diversion failed to achieve statistical significance for intraoperative (OR: $1.0, p=0.9)$ and in-hospital complications (OR: 1.0, $p=0.8$ ).

Conclusions: After RC, urinary diversion dictates no difference in intraoperative or in-hospital complications. However, fewer transfusions and a lower in-hospital mortality rate were recorded in OCUD patients. Therefore, broader use of OCUD after RC should be encouraged.
\end{abstract}

\section{UP-002}

Techniques and Agents Used to Conserve Blood During Radical Cystectomy: a Survey of the Society of Urologic Oncology Punjani, Nahid ${ }^{1}$; Lavallée, Luke T. ${ }^{2}$; Momoli, Franco ${ }^{3}$; Cagiannos, Ilias²; Morash, Christopher G. ${ }^{2}$; Fergusson, Dean ${ }^{3}$; Breau, Rodney $\mathrm{H}^{2}{ }^{2}$

${ }^{1}$ University of Ottawa, Ottawa, ON, Canada; ${ }^{2}$ University of Ottawa, Division of Urology, Ottawa, ON, Canada; ${ }^{3}$ Ottawa Hospital Research Institute, Ottawa, ON, Canada

Introduction and Objectives: Radical cystectomy may result in significant blood loss necessitating blood transfusions. The purpose of this study was to determine what intraoperative techniques and agents are currently used by uro-oncologists to prevent blood loss during radical cystectomy.

Methods: In August 2011, Society of Urologic Oncology members were solicited to complete an online survey. Respondents were asked to provide demographic information, state opinions on blood loss and transfusion, report techniques used to reduce blood loss and to estimate the proportion of cases where they used either systemic or local hemostatic agents. Results: Residents, fellows, and non-urologists were excluded leaving 86 staff urologists who perform radical cystectomies. Of the 86, $73(85 \%)$ had completed an uro-oncology fellowship in the United States, 57 (66\%) had been in practice for over 6 years, and $68(79 \%)$ perform over 10 cystectomies per year. Forty-nine $(57 \%)$ of respondents estimated that trans- fusions were performed in over $20 \%$ of patients and $4(5 \%)$ indicated that transfusions were performed in over $50 \%$ of patients. Few surgeons reported using CellSaver $(15 ; 17 \%)$, autologous blood banking $(16 ; 19 \%)$, or acute normovolemic hemodilution (22;26\%). Topical hemostatic agents were frequently administered with $61(71 \%)$ utilizing oxidized cellulose polymer (Sugicel $囚), 23(27 \%)$ absorbable gelatin sponge (Gelfoam $\left.{ }^{\circledR}\right), 44$ $(51 \%)$ gelatin and thrombin matrix (Floseal®), and $15(17 \%)$ thrombin and calcium chloride $($ Tisseel $\mathbb{R}$ ). Very few surgeons routinely used systemic antihemorrhagics with only $2(2 \%)$ reporting use of factor VII (Novoseven $($ ),

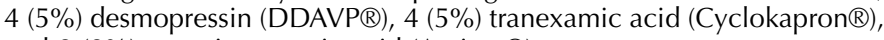
and $0(0 \%)$ to aminocaproic acid (Amicarß).

Conclusions: There is significant risk of blood loss requiring transfusion during radical cystectomy even among high volume uro-oncologists. Surgeons frequently use topical hemostatic agents and rarely use systemic anti-hemorrhagics.

\section{UP-003}

Should CT Urogram Be the First Radiological Investigation to Evaluate Visible Haematuria?

Akhter, Waseem; Sheikh, Mazher; Kilburn, Karen; Peedikayil, Abraham; Palit, Victor

University Hospital of North Tees and Hartlepool NHS Trust, Hartlepool, United Kingdom

Introduction and Objectives: General protocol for investigating visible haematuria patients are USS/IVU and flexible cystoscopy. However pooled sensitivity and specificity of CT urogram in diagnosis of urothelial tumors are $96 \%$ and $99 \%$ respectively (systematic review and meta-analysis EJR Feb 2010) which is better than IVU or USS. So CT urogram became the investigation of choice for visible haematuria at our haematuria clinic. The purpose of this study is to assess the effectiveness and outcome of CT urogram performed as the first radiological investigation for patients with visible haematuria.

Methods: This is a retrospective review of all CT urograms results at haematuria clinic from January 2009 to January 2010. Endoscopic findings were also noted in all patients.

Results: This study includes 266 patients with visible haematuria. A total of 76 patients had some abnormal urological findings in CT urogram (Table 1). 31 patients avoided flexible cystoscopy and proceeded directly for resection of bladder tumor/retrograde studies and ureteroscopy. 8 patients were referred to other clinicians for non-urological findings after normal flexible cystoscopy.

Conclusions: CT urogram should be the first line of imaging for visible haematuria as it avoids unnecessary investigations and helps plan effective management of haematuria patients.

Table 1. UP-003. CT urogram ( $=266)$; abnormal CT urogram $(n=76)$

\begin{tabular}{lc}
\hline RCC & $5(1.87 \%)$ \\
Upper TCC & $12(4.51 \%)$ \\
Bladder TCC & $33(12.40 \%)$ \\
Stones & $26(9.77 \%)$ \\
Other diagnoses & $8(3 \%)$ \\
\hline CT: computed tomography; RCC: renal cell carcinoma; TCC: transitional cell carcinoma. \\
\hline
\end{tabular}




\section{UP-004}

\section{Laparoscopic Robot-assisted Radical Cysto-prostatectomy} Bladou, Franck

McGill University, Jewish General Hospital, Montreal, QC, Canada Introduction and Objectives: Radical cysto-prostatectomy is indicated for non-metastatic infiltrative transitional cell carcinoma of the bladder. During the procedure, an extended lymph node dissection is performed with a control of the vesical and prostatic pedicles, a dissection of the recto-vesical plan and urethral diversion. Due to the anatomic situation of these organs in the deep pelvis, the standart open procedure has an early postoperative morbidity that exceeds a $30 \%$ rate and a long length of stay. Methods: A laparoscopic approach has been proposed for this procedure in order to limitate the per-operative blood loss and morbidity rate. It has been shown to be limited to experienced centres, due to the technical difficulty and demanding procedure. Robotic-assistance is an alternative of pure laparoscopic procedure and can be proposed to enhance the surgeon gesture in a limited anatomic area while keeping oncological safety. Results: The video shows a step-by-step laparoscopic robot-assisted radical cysto-prostatectomy, with an extended lymp node dissection from the aorto-iliac bifurcation, external iliac, obturator and internal iliac dissection. The 3-D surgical vision offered by the DaVinci technology allows a perfect vision of the surgical dissection. Vesical pedicles are cliped at the origin of the internal iliac vessels, allowing a dry dissection with limited blood loss. The complete dissection of the bladder is shown, followed by the control of the Santorini pedicle, urethral and ureteral transections. At the end of the procedure, a running suture is placed on the posterior lip of the urethra to prepare the ileo-urethral anastomosis that will be performed after the construction of the neo-bladder performed in an open approach. Conclusions: Radical cysto-prostatectomy can be performed with the same oncological safety than open procedure, equivalent procedure time and lower blood loss.

\section{UP-005}

\section{Survival Impact of Postoperative Expenditures by High Volume} Surgeons Following Definitive Surgery for Bladder Cancer

Sandhu, Gurdarshan; Nepple, Kenneth; Yang, Liu; Grubb 3 ${ }^{\text {rd }}$, Robert; Strope, Seth

Washington University School of Medicine, St. Louis, MO, United States Introduction and Objectives: Improved survival has been reported in high volume surgeons, but it is unknown whether variation exists in the outcomes of such surgeons based on surveillance patterns. We evaluated the postoperative expenditures of high volume surgeons and explored the association between these expenditures and survival.

Methods: Using SEER-Medicare records, we identified 2408 patients aged $\geq 66$ years with bladder carcinoma treated with definitive surgery from 1992 to 2005. Surgeons were defined as high volume for performance of $\geq 10$ cystectomies in the cohort. Geography and time (2005) standardized outpatient postoperative Medicare expenditures were evaluated for 2 years after surgery. High volume surgeons were stratified into quartiles by mean monthly postoperative expenditures, and survival for the quartiles was evaluated with the Kaplan-Meier method. Multivariable Cox proportional hazard regression models were used to estimate mortality hazard ratios by expenditure quartile.

Results: 29 of 833 surgeons were identified as high volume and had operated on 443 patients. Mean monthly postoperative expenditures by individual surgeon ranged from $\$ 41.19$ to $\$ 181.73$ amongst the high volume surgeons. No difference in cancer specific $(p=0.30)$ or overall survival $(p=0.09)$ was seen between the surgeon expenditure quartiles on Kaplan-Meier analysis. After adjusting for demographic, socioeconomic, comorbid, treatment, pathologic, hospital and surgeon factors no difference in mortality outcomes were seen between quartiles (Table 1).

Conclusions: In high volume surgeons, a broad range in postoperative management was observed, manifested by large differences in postoperative expenditures. Despite the increased cost of postoperative care, no difference in survival was seen between surgeon expenditure quartiles implying that improved outcomes in high volume surgeons may be more strongly related to the surgery itself than to strict postoperative surveillance.

\begin{tabular}{|c|c|c|c|}
\hline \multicolumn{4}{|c|}{ Cancer Specific Mortality } \\
\hline $\begin{array}{l}\text { Postoperative Expenditure } \\
\text { Quartile }\end{array}$ & HR & $95 \% \mathrm{Cl}$ & $p$ value \\
\hline $\begin{array}{l}\text { Average Monthly Expenditures } \\
\leq \$ 46.24\end{array}$ & Referent & - & - \\
\hline $\begin{array}{l}\text { Average Monthly Expenditures } \\
\$ 46.25-\$ 61.58\end{array}$ & 0.29 & $\begin{array}{c}0.08- \\
1.04\end{array}$ & 0.06 \\
\hline $\begin{array}{l}\text { Average Monthly Expenditures } \\
\$ 61.59-68.09\end{array}$ & 0.48 & $\begin{array}{c}0.20- \\
1.14\end{array}$ & 0.10 \\
\hline $\begin{array}{l}\text { Average Monthly Expenditures } \\
>\$ 68.09\end{array}$ & 0.63 & $\begin{array}{c}0.23- \\
1.75 \\
\end{array}$ & 0.38 \\
\hline \multicolumn{4}{|c|}{ Overall Mortality } \\
\hline $\begin{array}{l}\text { Postoperative Expenditure } \\
\text { Quartile }\end{array}$ & HR & $95 \% \mathrm{Cl}$ & $p$ value \\
\hline $\begin{array}{l}\text { Average Monthly Expenditures } \\
\leq \$ 46.24\end{array}$ & Referent & - & - \\
\hline $\begin{array}{l}\text { Average Monthly Expenditures } \\
\$ 46.25-\$ 61.58\end{array}$ & 0.49 & $\begin{array}{c}0.19- \\
1.32\end{array}$ & 0.16 \\
\hline $\begin{array}{l}\text { Average Monthly Expenditures } \\
\$ 61.59-68.09\end{array}$ & 0.96 & $\begin{array}{c}0.51- \\
1.83\end{array}$ & 0.91 \\
\hline $\begin{array}{l}\text { Average Monthly Expenditures } \\
>\$ 68.09\end{array}$ & 0.90 & $\begin{array}{l}0.40- \\
2.00\end{array}$ & 0.79 \\
\hline
\end{tabular}

\section{UP-006}

Is There a Role for the mTOR Pathway in Non-muscle Invasive Bladder Cancer?

Blais, Jean-Philippe; Hovington, Hélène; Brisson, Hervé; Lacombe, Louis; Caumartin, Yves

Centre Hospitalier universitaire de Québec, Quebec, QC, Canada

Introduction and Objectives: Non-muscle invasive bladder cancer (NMIBC) is associated with significant risk of recurrence and can progress to an invasive state. Numerous adjuvant intravesical therapies have been used in order to decrease the burden associated with this disease. The mammalian target of rapamycin (mTOR) pathway has been associated with oncogenesis in numerous cancers. We want to evaluate its implication in NMIBC and indirectly, its potential therapeutic role.

Methods: Immunohistochemical analyses were performed on first bladder tumors (BT) resected. We assessed mTOR overexpression with monoclonal antibodies. The different tumor specimens were classified according to staining percentage and intensity. Patients and tumors characteristics were reviewed from patients' charts. We attempted correlation of mTOR expression with clinical outcomes (recurrence and progression).

Results: BT with a high intensity staining were associated more often with T1 stage $(47.4 \%$ vs. $21.6 \%, p=0.04)$ and higher grade $(31.6 \%$ vs. $4.5 \%$, $p=0.0009)$ compared to BT with lower mTOR expression. The proportion of patients who experienced recurrence was more frequent in the high intensity expression group $(84.2 \%$ vs. $65.9 \%, p=0.17)$ as was also the risk of progression $(15.8 \%$ vs. $9.1 \% ; p=0.41)$. These results are unfortunately not statistically significant at this point, and for this reason, this study is still ungoing with inclusion of more patients.

Conclusions: Our preliminary results have demonstrated a trend between mTOR overexpression and risk of recurrence and progression in NMIBC. We need to continue the analysis with more patients in order to ascertain this potential pronostic relation. Through this study, we also hope to provide a rationale for the potential mTOR inhibitors utilization in NIMBC. 
UP-007

\section{Meta-analysis of the Efficacy and Safety of Darifenacin and} Other Anticholinergics for Managing Overactive Bladder

Elhilali, Mostafa'; Piwko, Charles'; Vincente, Colin ${ }^{2}$; Sabapathy, Suthacar ${ }^{2}$; Tu, Le Mai ${ }^{3}$; Valiquette, Luc ${ }^{4}$; Finarson, Thomas R. ${ }^{5}$; Ahmad, Asma ${ }^{6}$ ${ }^{1}$ McGill University, Royal Victoria Hospital, Montreal, QC, Canada; ${ }^{2}$ Pivina Consulting, Montreal, QC, Canada; ${ }^{3}$ FRCSC University of Sherbrooke, Sherbrooke, QC, Canada; ${ }^{4}$ FRCSC Université de Montréal, Montreal, QC, Canada; ${ }^{5}$ University of Toronto, Toronto, ON, Canada; ${ }^{6}$ Pivina Consulting, Mississauga, ON, Canada

Introduction and Objectives: Anticholinergics are frequently used to treat Overactive Bladder (OAB) symptoms which impacts quality of life (QoL). They cause adverse events, including dry mouth and constipation. The efficacy and safety of Darifenacin is reported to be similar to other anticholinergics with less cardio-vascular and cognitive adverse events (AE). Our objective was to conduct an updated meta-analysis comparing the efficacy, safety, and impact on QoL of Darifenacin and other anticholinergics (oxybutynin, tolterodine, solifenacin, and trospium chloride) for managing $\mathrm{OAB}$.

Methods: The search was conducted by 2 independent researchers on Medline, Embase, and Cinhal to identify RCTs reporting safety (dry mouth, constipation, and withdrawals) and efficacy (urgency incontinence episodes, number of micturitions frequency, urgency and total incontinence episodes, and volume voided per micturition) of darifenacin and other anticholinergics. Each researcher extracted data independently. Discrepant results were settled through consensus discussion. The metaanalysis was performed using a random effects model.

Results: The literature search resulted in 1,280 citations of which 41 RCTs contained sufficient data for analysis including 3 Darifenacin RCTs $(n=1226)$. All anticholinergics improved clinical outcomes from baseline with high responses to placebo. Trospium chloride and Darifenacin improved all efficacy outcomes compared to placebo. In addition Darifenacin improved QoL compared to placebo. All agents caused some dry mouth and constipation, with the lowest level of total withdrawals noted for Darifenacin.

Conclusions: This meta-analysis demonstrated that Darifenacin is similar in clinical efficacy and safety compared to other anticholinergics, but more effective in terms of QoL.

\section{UP-008}

Preoperative Sarcopenia Associated with Renal Function Outcomes in Patients Treated for Renal Masses by Extirpative Surgery

Hennessey, Katherine K.; Michael, Amanda; Elias, Rami; Kapoor, Anil; Shayegan, Bobby; Matsumoto, Edward; Mourtzakis, Marina; Di Sebastiano, Katie; Duivenvoorden, Helga; Pinthus, Jehonathan H.

McMaster University, Hamilton, ON, Canada

Introduction and Objectives: Patients with solid tumors and severe skeletal muscle depletion (sarcopenia) have reduced survival. In renal transplants, sarcopenia predicts poorer post-transplant graft and patient survival. Preoperative body mass index (BMI) has been found to be an independent predictor of renal function post-nephrectomy. We hypothesize that sarcopenia may also predict renal function following surgery. Methods: We examined the association between preoperative sarcopenia and postoperative estimated GFR (eGFR). Skeletal muscle area at the 3 rd lumbar vertebrae was measured on computed tomography, and analyzed using Slice-O-Matic software. Cut-offs for sarcopenia were set as per standards in the literature. The primary outcome was change in a modified eGFR postoperative, calculated using the Modification of Diet in Renal Disease Study Group equation.

Results: Mean BMI of the sample was 30.1 and mean age was 62.7. Mean preoperative GFR was 91.5 in the sarcopenic patients. The group included 5 open and 13 laparoscopic radical nephrectomies. 10/18 (56\%) were sarcopenic preoperative, revealing a high prevalence of sarcopenia in RCC patients. Mean change in 3 months eGFR was $-30.2 \%$ and $-27.5 \%$ in the sarcopenic and non-sarcopenic groups, respectively. Mean change in 12 months eGFR was $-29.5 \%$ and $-28.8 \%$ in the sarcopenic and non-sarcopenic groups, respectively. Unpaired t-test showed change in eGFR in the sarcopenic group greater than the non-sarcopenic, approaching significance $(p=0.1)$. A trend between preoperative sarcopenia and decreased postoperative renal function is evident.

Conclusions: Preoperative sarcopenia may be a predictor of renal failure post-nephrectomy. Sarcopenic patients have a lower GFR postoperative. This pilot study is ongoing as we collect data on 100 more patients. With a larger sample, we expect to detect a statistically significant decrease in postoperative eGFR in sarcopenics. Goals of surgery for renal tumors include preservation of function, and thus a nephron-sparing approach could be applied to sarcopenics.

\section{UP-009}

Renal Tumor Ablation for pT1 Lesions: Patterns of Recurrence and Follow-up Recommendations

Rutkowski, John; Muruve, Nic

Cleveland Clinic Florida, Weston, FL, United States

Introduction and Objectives: Renal tumor ablation has gained popularity for the treatment of pT1 lesions. We seek to determine the pattern and frequency of recurrence to optimize follow-up.

Methods: We retrospectively analyzed the records of consecutive patients who underwent renal tumor ablation at our institution from 6/2004 to 6/2011. Patients who underwent percutaneous RFA and laparoscopic cryoablation were included. Follow-up imaging studies and clinical notes were reviewed to identify patients who failed therapy. Failure was defined as persistent contrast enhancement or requirement for an additional procedure.

Results: A total of 94 ablations performed in 87 patients with appropriate follow-up were included in our study. Of 65 percutaneous RFAs, there were 11 failures. The average tumor size for failures was $3.5 \mathrm{~cm}$ (range $1.5-5.5 \mathrm{~cm}$ ). The average time to failure after treatment was 6 months (range 3-18 months). Failures were treated with repeat percutaneous RFA (6), partial nephrectomy (1) and no treatment (3). One patient developed biopsy proven metastases during follow-up. Of 29 laparoscopic cryoablations, there were 4 failures. The average tumor size for failures was 3.35 $\mathrm{cm}(2-4 \mathrm{~cm})$. The average time to failure after treatment was 15 months (range 6 - 30 months). Failures were treated with percutaneous RFA (3) and nephrectomy (1). The overall failure rate was $16.9 \%$ for percutaneous RFA and $13.7 \%$ for laparoscopic cryoablation.

Conclusions: Failure of tumor ablation appears to occur early and in larger tumors suggesting technical failures rather than recurrent disease. We recommend follow-up consist of a CT scan at 6 months to document proper ablation and a second one at either 12 or 18 months from the time of treatment since most failures occur then and progression is rare. Longer intervals can be considered for patients with tumors less than 3 $\mathrm{cm}$ as failure in this group was less frequent.

\section{UP-010}

Cystatin C for Early Detection of Acute Kidney Injury after Laparoscopic Partial Nephrectomy

Al Esawi, Anwar; Nadeau, Geneviève; Caumartin, Yves; Bergeron, Alain; Guimezap, Jackson; Fradet, Vincent; Caron, André; Dujardin, Thierry; Fradet, Yves; Lacombe, Louis

CHUQ-Laval University, Quebec, QC, Canada

Introduction and Objectives: Partial nephrectomy is the gold standard for the treatment of small renal tumors. Warm ischemia time is one the factor associated with kidney damage after partial nephrectomy but the safe clamping time is still unknown. There is a need to identify biomarkers that would improve the early detection of acute kidney injury (AKI). The objective of this study was to assess whether cystatin $\mathrm{C}$ levels obtained at specific timepoints during laparoscopic partial nephrectomy (LPN) could be early predictors of AKI.

Methods: Twenty-five patients participated in this study. Plasma samples were collected preoperatively, 5 min before clamping, 5, 20 and 120 min post-declamping and on the day following surgery. Plasma creatinine and cystatin $C$ were measured by enzymatic and enzyme-linked immunosorbent assays, respectively. Associations between levels of cys- 
tatin C, creatinine, and AKI-related data were measured using Pearson's correlation statistic.

Results: Clamping time varied between 16 and 44 minutes. Postoperatively, only four of the 25 patients had a 1.5 to 1.9 -fold increase in serum creatinine from baseline and were identified with stage $1 \mathrm{AKI}$ according to the AKIN classification. Postoperative cystatin C levels compared to baseline were increased in $13(52 \%)$ of the patients. The differences between post- and preoperative cystatin $\mathrm{C}$ and creatinine values were highly correlated $(r=0.7697 ; p<0.0001)$. Four patients had a $\geq 1.25$-fold increase in cystatin $C$ levels from baseline and three of these were stage I AKI patients. Intraoperative cystatin C levels were increased from baseline in $12(48 \%)$ of the patients; however no association was found with parameters associated to AKI.

Conclusions: High increase in postoperative cystatin $\mathrm{C}$ levels from baseline may help identify patients with AKI following LPN. However, intraoperative cystatin $\mathrm{C}$ levels do not seem to be predictors of $\mathrm{AKI}$ in this small cohort of patients.

\section{UP-011}

Ex-vivo Partial Nephrectomy with Autotransplant to Treat Complex Renal Tumors: Case Report and Review of the Literature Nayak, Jasmir; Archambault, Jason; Koulack, Joshua; McGregor, Thomas B. University of Manitoba, Winnipeg, MB, Canada

Introduction and Objectives: Nephron sparing surgery has become the gold standard for patients with tumors in solitary kidneys, bilateral renal masses, genetic renal masses, or patients with chronic renal dysfunction or risk of future renal impairment. We describe the use of nephrectomy followed by ex-vivo partial nephrectomy, bench renography, and then autotransplantation to treat a complex renal mass in a solitary kidney.

Methods: We present our case of management of a complex renal mass in a solitary kidney with 1 year follow-up. The medical literature for ex-vivo partial nephrectomy was then reviewed and analyzed.

Results: Radical nephrectomy was performed through a flank incisionand followed by immediate renal cooling and perfusion with Histidinetryptophan-ketoglutarate solution, Ex-vivo partial nephrectomy and renography were then performed, followed by successful autotransplantation in the native renal bed. Cold ischemia time was less then forty minutes and warm ischemia time negligible. Estimated blood loss was $100 \mathrm{cc}$, with no intra-operative complications. Postoperative course was uneventuful aside from a urinoma that resolved with drain placement. Preoperative $\mathrm{Cr}$ was $116 \mathrm{umol} / \mathrm{L}$ and peaked at $165 \mathrm{umol} / \mathrm{L}$ postoperatively. This patient is disease free with a stable creatinine of $130 \mathrm{umol} / \mathrm{L}$ at 1 year follow-up. In accordance with the limited number of reports in the literature, our experience suggests that good outcomes can be achieved with this technique. Conclusions: Ex-vivo partial nephrectomy and autotransplantation is a viable option for patients with a complex renal mass in a solitary kidney. The ex-vivo nature of the procedure allows for excellent exposure, a bloodless field and complex renography.

\section{UP-012}

LKB1 Drives Adiponectin Receptor 1 Expression in Clear Cell Renal Cell Carcinoma: from Tumor Development to Disease Characteristics

Zareba, Piotr; Duivenvoorden, Wilhelmina; Beatty, Laura; Lhotak, Sarka; Lu, Jian-Ping; Austin, Richard; Daya, Dean; Pinthus, Jehonathan H.

McMaster University, Hamilton, ON, Canada

Introduction and Objectives: Evidence suggests that RCC development is linked to dysregulation of metabolic pathways. The AMPK/mTOR axis is the main energy-sensoring pathway, which, upon activation, results in mTOR inhibition. AMPK is regulated mainly by LKB1. We previously found that adiponectin, through its receptor AdipoR1, induces AMPK activation and consequently tumor suppression. We thus examined the status of AdipoR1 in cCRCC.

Methods: Specimens of ccRCC from 25 patients and their surrounding normal renal parenchyma (NRP) were analyzed for the expression of LKB1 and AdipoR1 by Western blot. A tissue microarray containing tissues from 239 ccRCC patients was stained for AdipoR1 or LKB1. Using
ImageScope software, the staining intensity ( $\mathrm{H}$-score) was determined. Associations between AdipoR1 $\mathrm{H}$-scores and tumor grade and stage were tested using linear regression. In vitro the AdipoR1-LKB1-AMPK pathway was examined using stable knockdowns of human ccRCC CRL-1932 cells for AdipoR1 or LKB1. Western blot was used to detect AMPK activation and mTOR inhibition.

Results: The expression of LKB1 was significantly reduced in ccRCC compared to NRP. AdipoR1 expression was lower (mean reduction $80 \%$ ) in 24/25 cCRCC compared to NRP. Yet, the expression of both LKB1 and AdipoR1 correlated positively with tumor grade and stage. In vitro AdipoR1 protein expression in ccRCC cells was reduced with LKB1 knockdown. However, LKB1 expression was not reduced with AdipoR1 knockdown. Adiponectin-mediated AMPK activation and mTOR inhibition were disrupted with both AdipoR1 and LKB1 knockdown.

Conclusions: The expression of both AdipoR1 and LKB1 is critical for AMPK activation and mTOR inhibition. While ccRCC development was associated with lower LKB1 and hence AdipoR1 expression, the expression of these metabolic mediators correlated reciprocally with cCRCC grade and stage, once the tumor developed. Our results may be explained by compensatory metabolic changes occurring in tumor progression.

\section{UP-013}

Kidney Cancer Survivorship Survey: Gap Between Urologist and Survivor Perceptions

Basiuk, Joan ${ }^{1}$; lewett, Michael A.S. ${ }^{2}$; Maskens, Deborah ${ }^{3}$; Canil, Christina ${ }^{4}$ ${ }^{1}$ Kidney Cancer Canada, Toronto, ON, Canada; ${ }^{2}$ Princess Margaret Hospital, University of Toronto, Toronto, ON, Canada; ${ }^{3}$ Kidney Cancer Canada, Guelph, ON, Canada; ${ }^{4}$ The Ottawa Hospital Cancer Centre/ University of Ottawa, Ottawa, ON, Canada

Introduction and Objectives: The number of survivors with kidney cancer $(\mathrm{KC})$ in Canada is growing as a result of increasing incidence, earlier diagnosis and improvements in therapy. Yet, the recently published "Investment in Research on Survivorship and Palliative and End-of-Life Care", reported no investment in KC survivorship research between 20052008. Kidney Cancer Canada (KCC) has conducted the first Canadian KC survivorship survey. The availability of information pertaining to survivorship, the extent to which it is communicated to patients and the interest in more formalized survivorship care plans were the focus of the survey. Methods: Two comparable, online surveys (one for physicians and another for patient/caregivers) were developed to measure knowledge levels regarding KC survivorship issues. Urologists and patient/caregivers across Canada were invited to participate. Forty urologists, $276 \mathrm{KC}$ patients and 45 caregivers of KC patients diagnosed at stages 1 through 3 completed surveys.

Results: Urologists reported that they communicated information regarding stage $(100 \%)$, grade $(98 \%)$, tumor size $(85 \%)$ and cell subtype $(83 \%)$ to their KC patients. In contrast, KC patients/caregivers reported much lower rates for receiving information on their stage $(62 \%)$, grade $(53 \%)$, tumor size $(80 \%)$ and cell type $(63 \%)$. Furthermore, nearly half $(46 \%)$ of those affected by KC reported that they received no information from their urologist about possible adverse effects of treatment such as increased risks of chronic kidney disease or hypertension. However, both groups supported the need for a medically endorsed website that would provide patients with an individualized survivorship care plan.

Conclusions: KCC's Survey has identified a gap between Canadian urologist and KC survivor/caregiver perceptions about the provision of perioperative information. However, both groups supported the development of a kidney cancer survivorship care plan. 


\section{UP-014}

Comparison of Renal Function Outcomes Between Laparoscopic Partial Nephrectomy with Early Unclamping versus Laparoscopic Partial Nephrectomy with Zero Ischemia

Tse, Richard ${ }^{1}$; Karreman, Erwin²; Maslany, Jogann'; Knaus, Russel ${ }^{1}$; Tse, Edward $^{1}$

${ }^{1}$ University of Saskatchewan, Regina, SK, Canada; ${ }^{2}$ University of Saskatchewan, Regina Qu'Appelle Health Region, Regina, SK, Canada Introduction and Objectives: Limiting warm vascular occlusion time to less than 20-30 minutes during partial nephrectomy is vital to minimize renal ischemic damage. Although, both early unclamping and zero ischemic partial nephrectomy can achieve this time-restraint, only limited data exists comparing their renal function outcomes. We aim to compare renal function outcomes of these two approaches by measuring pre- and postoperative eGFR and post operative MAG 3 renal scans.

Methods: Between September 2009 to October 2011, 34 consecutive patients underwent laparoscopic partial nephrectomy with early unclamping with mean clamp time of 19.04 minutes. This was followed by 30 consecutives patients undergoing laparoscopic partial nephrectomy with zero ischemia. All cases were performed by a single surgeon. We retrospectively reviewed records of all 64 patients. Patients with solitary kidneys and patients with significant renal parenchyma disparity on preop CT scan were excluded from the study. Demographics, tumor pathology, surgical margins, perioperative complications, pre- and postoperative eGFR and postoperative MAG 3 Scans were compared between both groups. Results: See Table 1.

Conclusions: Although no statistically significant differences were found, the difference between the two groups was more profound for the MAC 3 Scan variables. Associated r-values show small to medium-sized effects in this regard, which point towards a trend of improved renal function preservation in the zero ischemic group. Larger multicentre trials are required for future research in this area.

\section{UP-015}

Reduction of Renal Parenchymal Volume and Renal Function in Patients after Partial Nephrectomy for Renal Cell Carcinoma (RCC)

Ajzenberg, Henry ${ }^{1}$; Legere, Laura ${ }^{1}$; Satkunasivam, Raj $^{2}$; Finelli, Antonio ${ }^{1}$; Kachura, John ${ }^{3}$; O'Malley, Martin ${ }^{3}$; Tuchscherer, Paul ${ }^{4}$; Quinn, Paul ${ }^{4}$; Choi, Jung'; Jewett, Michael A.S. ${ }^{1}$

${ }^{1}$ Princess Margaret Hospital, Toronto, ON, Canada; ${ }^{2}$ University of Toronto, Toronto, ON, Canada; ${ }^{3}$ Toronto General Hospital, Toronto, ON, Canada; ${ }^{4}$ Toronto Western Hospital, Toronto, ON, Canada

Introduction and Objectives: Nephrectomy and partial nephrectomy (PN) for early-stage RCC produce similar oncological outcomes. However, the loss of normal kidney tissue, or renal parenchymal volume (RPV) may increase the risk of renal dysfunction. The use of PN is increasing and the extent of renal dysfunction attributable to RPV loss is not fully understood. Methods: 31 patients who underwent PN for RCC at the University Health Network were retrospectively studied. Demographic, surgical, and renal functional data were extracted from patient charts. Glomerular filtra- tion rate (GFR) was estimated using the Modification of Diet in Renal Disease (MDRD) equation and plasma creatinine concentrations. Changes in pre- and postoperative GFR were quantified ( $\triangle$ GFR). RPV loss ( $\triangle R P V)$ was determined using pre- and postoperative CT or MR scans on Vitrea volumetric software. Non-tumor kidney tissue on each axial slice was manually traced. A correlation between $\triangle R P V$ and $\triangle$ GFR was assessed. Results: There were 18 males and 13 females with a mean age of 57 years (range 28-80). Mean tumor diameter was $3.6 \mathrm{~cm}$ (range 1.3-7.5). Mean pre- and postoperative RPV was $160.9( \pm 33.7$, range $81.4-229.5)$ and $144.2 \mathrm{~mL}( \pm 36.8$, range $61.6-199.8)$, respectively. Mean $\triangle \mathrm{RPV}$ was $-10.6 \%( \pm 14.8)$ or $-16.7 \mathrm{~mL}( \pm 25.1$, range $-87.1-25.1)$. Mean preoperative GFR was $86.3 \mathrm{ml} / \mathrm{min} / 1.73 \mathrm{~m}^{2}( \pm 20.1$, range $53.2-128.3)$. Twelve months postoperatively, mean GFR was $80.1 \mathrm{ml} / \mathrm{min} / 1.73 \mathrm{~m}^{2}( \pm 26.5$, range 37.7 144.91). Mean $\Delta$ GFR was $-8.1 \%( \pm 20.9)$ or $-6.6 \mathrm{ml} / \mathrm{min} / 1.73 \mathrm{~m}^{2}( \pm 18.1$, range -68.6-25.4). Using a linear regression, we found no correlation between $\triangle$ RPV and $\triangle$ GFR $\left(r^{2}=.002, p=0.812\right)$.

Conclusions: PN does lead to renal dysfunction, but RPV loss does not fully account for this. We found no correlation between $\triangle R P V$ and $\triangle G F R$. Patient comorbidity, ischemia/reperfusion injury, and compensatory renal reserve may have a larger impact on GFR than minimal loss of RPV with $\mathrm{PN}$. We are increasing our sample to further explore this relationship.

\section{UP-016}

\section{Results of a Survey on the Management of Upper Tract Urothelial} Cancer

Zappavigna, Christopher; Rowe, Neal; Morash, Christopher G.; Breau, Rodney H.; Cagiannos, Ilias

University of Ottawa, Division of Urology, Ottawa, ON, Canada Introduction and Objectives: The importance of regional lymphadenectomy has been well established in the management of bladder cancer. Considerable uncertainty exists regarding its role and utility in the treatment of upper urinary tract transitional cell carcinoma (UTTCC). A recent study has showed that the incidence of lymphatic involvement varied according to stage and grade. Our objective is to survey, study, examine and discuss the current management of UTTCC in Canada. Specically, what are the current practices for the management of UTTCC and when do we utilize lymphadenectomy?

Methods: A 6-page survey was created and sent electronically to all Canadian Urological Association (CUA) members. Data collected include physician demographics, UTTCC management practices and current practices for monitoring tumor recurrence and postoperative renal function. We studied the current practices for the management of UTTCC with a specific focus on lymphadenectomy. Moreover, we will also compile and evaluate data based on the current practices for monitoring tumor recurrence and postoperative renal function.

Results: A total of 27 urologists responded. $32 \%$ of respondents would do a lymphadenectomy for Ta/Tis/T1 disease and $64 \%$ would do a lymphadenectomy for $\mathrm{T} 2$ or greater disease. If presented with survival data favoring Retroperitoneal Lymph Node Dissection (RPLND) for Ta/Tis/T1 disease, $55 \%$ would change their management and $86 \%$ would change their management for $\mathrm{T} 2$ disease.

Table 1. UP-014

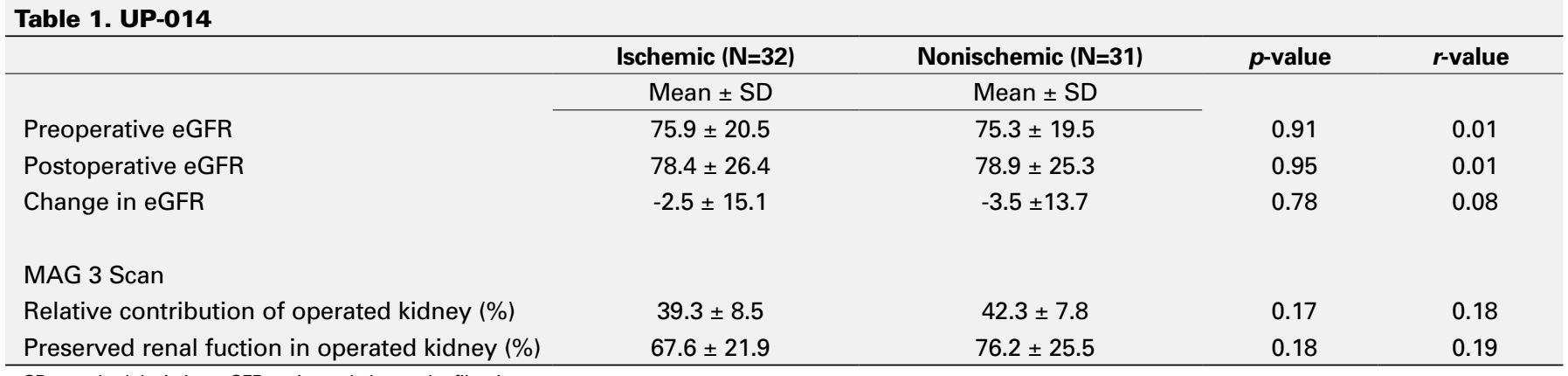

SD: standard deviation; eGFR: estimated glomerular filtration rate. 
Conclusions: Clearly, there is much debate over role of lymphadenectomy in this disease. The relatively low frequency of these lesions and the lack of prospective randomized trials do not permit absolute conclusions about treatment impact on outcomes. Furthermore, it is questionable whether a randomized controlled trial is feasible. Overall, our results suggest the need for a well-defined role for role of lymphadenectomy in the management of upper tract urothelial carcinoma.

\section{UP-017}

Management of Collecting Duct Carcinoma: a Systematic Review, Management Approach, and Case Series

Dason, Shawn'; Sheridan-lonah, Anna ${ }^{1}$; Allard, Christopher'; Kajal, Babita $^{2}$; Aziz, Tariq ${ }^{2}$; Gill, Jaskirat ${ }^{3}$; Jamshaid, Hira ${ }^{3}$; Kapoor, Anil ${ }^{1}$

'Division of Urology, McMaster University, Hamilton, ON, Canada ${ }^{2}$ Department of Pathology and Molecular Medicine, McMaster University, Hamilton, ON, Canada; ${ }^{3}$ McMaster University, Hamilton, ON, Canada Introduction and Objectives: Collecting duct carcinoma (CDC) is a rare subtype of renal carcinoma. It is aggressive, presents symptomatically at an advanced stage, and has a poor prognosis. Little is known on what constitutes optimal management of CDC. The aim of this study is to develop an evidence-based approach to managing CDC.

Methods: Ovid Medline, The Cochrane Library, EMBASE, MacPLUS FS and conference proceedings (via Web of Science) were searched to identify studies relevant to the management of CDC. A systematic search strategy was developed and applied. Included studies had a minimum of 10 subjects receiving a single intervention. Series in which an evaluation of therapeutic effectiveness was not possible were excluded. An algorithm based on this review for approaching multidisciplinary CDC management is then presented. Four consecutive cases of CDC treated at our tertiary institution between 2006 and 2010 are then related to this algorithm.

Results: Our systematic review identified 3 studies relevant to the management of CDC. Firstly, a gemcitabine/cisplatin or carboplatin (GC) regimen resulted in a $26 \%$ partial or complete response rate in a phase II study of 23 patients with metastatic CDC. Two additional studies indicated that 49

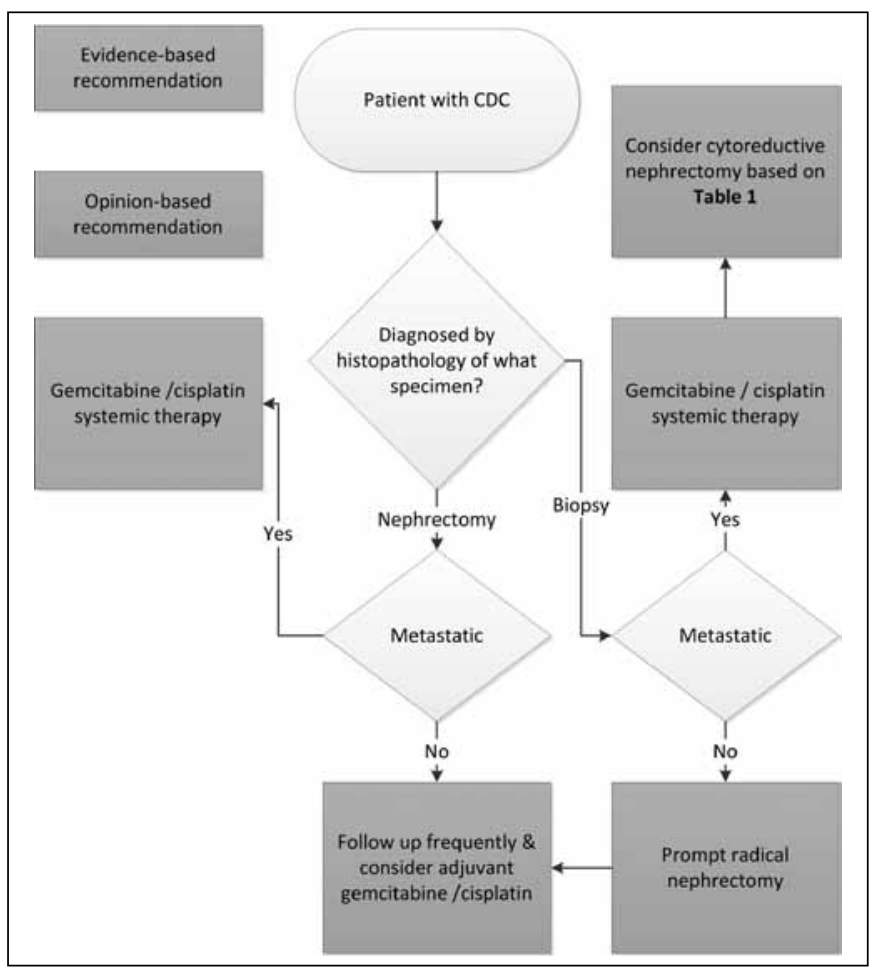

patients treated with immunotherapy achieved no response. A management algorithm based on these findings is presented in Figure 1. Four cases of advanced CDC are then reported. Two patients were unresponsive to MVAC therapy. Cytoreductive nephrectomy $(\mathrm{CN})$ was performed in 2 patients. Performance status and survival were uniformly poor. Management of these cases in relation to our algorithm (Fig. 1) is discussed.

Conclusions: CDC responds to systemic therapy similarly to urothelial carcinoma. Our review suggests that the current standard of care for metastatic CDC is a (GC) regimen. A framework for applying CDC management principles and considering $\mathrm{CN}$ based on this review is provided and applied to the 4 reported cases.

\section{UP-018 - WITHDRAWN}

\section{UP-019}

\section{Improving Outcomes Through the Development of Quality} Indicators (QI) in Renal Cell Cancer (RCC)

Finelli, Antonio $;$; Bjarnason, Georg ${ }^{2}$; Black, Peter $^{3}$; Cagiannos, Ilias ${ }^{4}$; Heng, Daniel $^{5}$; Kapoor, Anil ${ }^{6}$; Kollmannsberger, Christian ${ }^{7}$; Mohammadzadeh, Forough $^{8}$; Moore, Ronald ${ }^{9}$; Soulieres, Denis ${ }^{10}$; Tanguay, Simon ${ }^{11}$; Venner, Peter ${ }^{12}$; Jewett, Michael A.S. ; Rendon, Ricardo ${ }^{13}$; Wood, Lori' ${ }^{13}$

${ }^{1}$ Princess Margaret Hospital (UHN), Toronto, ON, Canada; ${ }^{2}$ Sunnybrook Odette Cancer Centre, Toronto, ON, Canada; ${ }^{3}$ Department of Urological Sciences, University of British Columbia, Vancouver, BC, Canada; ${ }^{4}$ University of Ottawa, Ottawa, ON, Canada; ${ }^{5}$ Tom Baker Cancer Centre, University of Calgary, Calgary, $\mathrm{AB}$, Canada; ${ }^{6}$ Juravinski Cancer Centre, Hamilton, ON, Canada; 'BCCA Vancouver Cancer Centre, UBC, Vancouver, BC, Canada; ${ }^{8} \mathrm{UHN}$, Toronto, ON, Canada; ${ }^{9}$ Department of Urology, University of Alberta, Edmonton, $\mathrm{AB}$, Canada; ${ }^{10}$ Department of Medicine, Université de Montréal, Montreal, QC, Canada; ${ }^{11}$ McGill University Health Centre, McGill University, Montreal, QC, Canada; ${ }^{12}$ Cross Cancer Institute, University of Alberta, Edmonton, AB, Canada; ${ }^{13}$ QEII HSC, Dalhousie University, Halifax, NS, Canada

Introduction and Objectives: Optimal quality of care is needed for ideal outcomes, and QI are used to measure quality of care. In RCC, there is a lack of information defining such optimal care. This is especially important as RCC care becomes more complex, with ongoing advances requiring greater expertise. The goal of the study was to identify QI for RCC across the disease spectrum from presentation to palliation.

Methods: A multidisciplinary expert panel of 13 urologic and medical oncologists from across Canada reviewed potential QI. These QI were identified from a systematic literature review. Panel members were also asked to suggest additional potential QI. A modified Delphi technique was used to select QI that were relevant and practical to RCC; this technique incorporated 2 email questionnaires and 1 in-person meeting.

Results: From 233 literature citations, 34 possible QI were identified; 24 additional potential QI were suggested. A final set of 23 QI was established. These are distributed across the RCC disease spectrum as follows (number of $\mathrm{QI}$ in parentheses): screening (1), diagnosis/prognosis (3), surgical management of localized disease (6), surgical management of advanced disease (3), systemic therapy (5), and follow-up (3). These 21 QI focused mostly on the treatment of RCC. In addition, two QI related to survival outcomes (overall and progression-free) were selected. Examples of QI include: the proportion of patients undergoing partial nephrectomy for tumors $<4 \mathrm{~cm}$ and the proportion of patients with advanced disease who are assessed by a multidisciplinary genitourinary cancer team. The final 23 QI selected will be presented in detail.

Conclusions: A systematic, consensus-based approach was used to determine relevant QI in RCC care. These 23 QIs will provide a means of evaluating the quality of RCC care in an effort to improve outcomes in our patients. The next step will be to establish a means of measuring each of these QI based on defined or yet to be defined benchmarks.

Fig. 1. UP-017. 


\section{UP-020 \\ Rising Incidence of Upper Tract TCC (UTTCC) in Kettering, United Kingdom}

Khan, Faisal ${ }^{1}$; Payne, David²; Al-Sudani, Mohammed ${ }^{2}$; Khan, Zeb²; England, Roland ${ }^{2}$

${ }^{1}$ Freeman Hospital, Newcastle, United Kingdom; ${ }^{2} \mathrm{NHS}$, Kettering, United Kingdom

Introduction and Objectives: Tumors of the renal pelvis are rare and account for approximately $10 \%$ of all renal tumors and approximately $5 \%$ of all urothelial tumors. Ureteral tumors are even more uncommon, occurring with one quarter the frequency of renal pelvis tumors. In our hospital we experienced rising incidence of UTTCC as well increase in ureteric TCC. To confirm this we retrospectively analysed the data.

Methods: We collected our data retrospectively from 2005 to August 2011. The cases were identified via theatre, histopathology and MDT meeting records. We analyzed known risk factors, investigations, treatment, final histology and associated bladder tumor.

Results: Total of 41 patient were diagnosed with UTTCC between 2005 to March 2011. Most of the patients were male (33) with male to female ratio of approx 8:1. Mean age at Ist presentation was 64 years (range 42 to 92 years). Most of the patients were either smoking actively or had stopped smoking $(n=28)$. The analysis revealed rising incidence of UTTCC in Kettering as shown in table form. Of note we also recorded rising incidence of Ureteric TCC then pelvic TCC in these patients.

Conclusions: Our project showed rising incidences of UTTCC especially ureteric tumors. We postulate the rising trend could be secondary to combination of strong presence of leather and service industries in Kettering area or secondary to improved diagnostics tests.

\section{UP-021}

The Fer Kinase, a Nuclear Effector of Growth-promoting Factors Favoring Castration-resistant Prostate Cancer

Rocha, Joice ${ }^{1}$; Zouanat, Fatima'; Benidir, Tarik'1 Z Zoubeidi, Amina²; Hamel, Lucie $^{1}$; Scarlata, Eleonora ${ }^{1}$; Aprikian, Armen ${ }^{1}$; Chevalier, Simone ${ }^{1}$ ${ }^{1}$ Urologic Oncology Research Group, McGill University, Montreal, QC, Canada; ${ }^{2}$ The Vancouver Prostate Cancer, University of British Columbia, Vancouver, BC, Canada

Introduction and Objectives: Death from prostate cancer (PC) arises from castration-resistant (CR) metastatic disease, developing from an increasing cell ability to survive and thrive in response to a variety of growth-promoting factors (GFs) signaling through tyrosine kinases (TKs). The androgen receptor (AR) itself has appeared as a TK substrate in PC cells responding to epidermal growth factor (EGF), interleukin (IL)-6 and even androgens. As we reported that the Fer TK controls IL-6 signaling, we aimed to verify if Fer further contributes to aberrant signaling by additional GFs controlling AR activation in PC.

Methods: LNCaP cell survival/growth and death were measured by MTT assays and propidium iodide $(\mathrm{PI})$ staining, respectively. Fer down-regulation was achieved via siRNAs and PSA was measured by Real Time PCR. Tyrosine (Y) phophorylation, protein interactions and cellular localization were assessed.

Results: Fer was responsible for the LNCaP cell response to IL-6, since fer siRNA reduced growth by $90 \%$. Fer also controlled 50\%, 44\%, 36\% and $32 \%$ of the response to FBS (fetal bovine serum), R1881 (androgens), Insulin-like (I) GF-1 and EGF, respectively. Fer knockdown also resulted in $40 \%$ less cells when cultured without growth stimuli. Cell death was confirmed by PI staining. Moreover the full R1881 and IL-6 PSA response depended on Fer. IL-6 and R1 881 were most potent to increase Fer and AR $\mathrm{pY}$-levels along with their nuclear accumulation as observed in prostate tumors. AR pY-levels were modulated by Fer, both within cells and in kinase assays. Finally, Y223 appeared as a novel functional site preferred by the Fer TK and this AR motif was involved in the interaction with the Fer-SH2 domain.

Conclusions: The up-regulated nuclear Fer in PC appears to intervene in pathways triggered by several GFs contributing to aberrant signaling in CRPC.

\section{UP-022}

Single Photon Emission Computed Tomography/CT Imaging of Metastases Using Prostate Specific Membrane Antigen Antibody Chevalier, Simone'; Derbekyan, Vilma ${ }^{1}$; Scarlata, Eleonora'; Moffett, Serge ${ }^{2}$; Hamel, Lucie ${ }^{1}$; Zouanat, Fatima ${ }^{1}$; Aprikian, Armen ${ }^{1}$; Anidjar, Maurice ${ }^{1}$

${ }^{1}$ McGill University Health Centre Research Institute, Montreal, QC, Canada; ${ }^{2}$ ProScan, Montreal, QC, Canada

Introduction and Objectives: Current imaging methods to detect prostate cancer (PC) metastases (mets) lack sensitivity and specificity. Reliable cancer-imaging modalities are needed. Prostate Specific Membrane Antigen (PSMA) is an attractive target for molecular imaging in virtue of its distribution and overexpression in castrate-resistant (CR) PC. We aimed to test a labeled monoclonal antibody $(\mathrm{mAb})$ directed against an extracellular subdomain of human and canine PSMA to detect mets in the dog prostate cancer (DPC)-1 model by single photon emission computed tomography (SPECT)/CT imaging.

Methods: DPC-1 cells were implanted in immuno-suppressed (cyclosporine) dogs $(n=5)$. SPECT/CT was repeated during follow-up, 2 days after i.v. injection of ${ }^{111}$ Indium(In)-PSMA mouse mAb. In some instances,

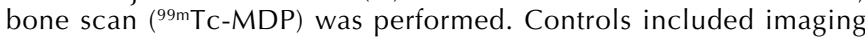
prior ${ }^{99 m}$ Tc-MDP, ${ }^{111}$ In-PSMA) and post ( ${ }^{111}$ In-mouse immunoglobulins) DPC-1 implantation. The prostate, sacroiliac lymph nodes (LNs), lungs and selected bone segments were harvested for gamma counting and pathological analyses.

Results: Four dogs developed prostate tumors and mets in LNs and lungs; 3 had bone mets. SPECT revealed uptake of ${ }^{111}$ In-PSMA radiotracer in mets, yet DPC-1 tumors in the prostate remained negative. Mets were imaged as early as by 6-8 weeks and grew during follow-up. CT fusion images indicated enlarged LNs, often necrotic at necropsy, and for which tracer accumulation and tumor positivity were confirmed. Uptake of radiotracer was also detected in lungs and bones (one case studied), confirming a positive bone scan. Controls were negative.

Conclusions: Molecular imaging by SPECT/CT with ${ }^{111}$ In-PSMA radiotracer was proven efficient and specific to detect soft tissue and bone mets in the pre-clinical DPC-1 model closely mimicking CRPC, implying feasibility in the clinical setting.

\section{UP-023}

PSA Bounce Following Prostate Brachytherapy for Clinically Localized Prostate Adenocarcinoma: a Single Institution Study with Minimum Three Years Follow-up

Nayak, Jasmir; Ong, Aldrich; Bews, Jeff; Chowdhury, Amit; Drachenberg, Darrel

University of Manitoba, Winnipeg, MB, Canada

Introduction and Objectives: Prostate specific antigen (PSA) is a sensitive serological marker of outcome following prostate brachytherapy. Following brachytherapy, PSA levels may transiently rise in a phenomenon known as PSA bounce (PB). We report on PB following permanent radio-iodine $\left({ }^{125} \mathrm{I}\right)$ brachytherapy and correlate PB with both clinical and dosimetric variables.

Methods: We analyzed 145 patients with clinically localized, T1-2c N0 $\mathrm{MO}$ prostate adenocarcinoma treated with brachytherapy with a minimum follow-up of 3 years. Six different PSA thresholds were used to define PB: a increase of $\geq 0.1 \mathrm{ng} / \mathrm{ml}$ (definition I), $\geq 0.2 \mathrm{ng} / \mathrm{ml}$ (definition II), $\geq 0.3$ (definition III) $\mathrm{ng} / \mathrm{ml}, \geq 0.4 \mathrm{ng} / \mathrm{ml}$ (definition IV), $\geq 0.5 \mathrm{ng} / \mathrm{ml}$ (definition V) and $\geq 1.0 \mathrm{ng} / \mathrm{ml}$ (definition $\mathrm{VI}$ ) with spontaneous return to spre-bounce levels. Biochemical failure (BF) was defined according to the American Society for Therapeutic Radiology and Oncology Phoenix definition of a rise of $\geq 2 \mathrm{ng} / \mathrm{mL}$ above the nadir.

Results: The median follow-up was 52.2 months (range: $36.1-74.8$ months). Using definitions I, II, III, IV, V and VI, a PB occurred in $44 \%$, $35 \%, 29,25 \%, 19 \%$ and $4 \%$ of patients respectively. The mean time until PB was $14.8-16.4$ months, the duration was $18.9-22.5$ months, and the magnitude was $0.7-3.2 \mathrm{ng} / \mathrm{mL}$. BF occurred in 15 patients $(10.3 \%)$ and was detected in $6.3 \%, 7.8 \%, 9.5 \%, 8.3 \%, 10.7 \%$ and $33.3 \%$ of PB 
patients using definitions I, II, III, IV, V and VI respectively. Nine patients $(6.2 \%)$ had true failure. All definitions of PB occurred earlier than BF $(p<0.05)$. Univariate and multivariate analysis revealed that age $<65$ and Gleason sum $\leq 6$, were statistically significant predictors of PSA bounce for all definitions, and percent positive biopsies $(<25 \%)$ for definitions I to IV. Conclusions: PB is a common phenomenon post-brachytherapy. Age $<65$, Gleason sum 6 and lower volume disease are predictors of PB. The time to first PSA rise can help to distinguish between PB and BF.

\section{UP-024}

Is Perineural Invasion in Prostate Biopsies Associated with Adverse Pathological Outcomes? Old Paradigm Revisited

Margel, David ${ }^{1}$; Elharram, Malik ${ }^{1}$; Finelli, Antonio ${ }^{1}$; Zlotta, Alexandre R. ${ }^{1}$; Trachtenberg, John'; Evans, Andrew ${ }^{2}$

${ }^{1}$ Division of Urology, Department of Surgical Oncology, University Health Network, University of Toronto, Toronto, ON, Canada; ${ }^{2}$ Department of Pathology, University Health Network, University of Toronto, Toronto, ON, Canada

Introduction and Objectives: To determine the role of perineural invasion (PNI) on prostate biopsy in predicting adverse findings at radical prostatectomy in a recent cohort of screen detected prostate cancer.

Methods: We analyzed 1041 consecutive patients from a prospectively maintained database. Prostate cancer was diagnosed in 470, and 138 of these patients underwent radical prostatectomy. Pathological specimens were examined, and perineural invasion was identified as carcinoma tracking along or around a nerve in the perineural space. We investigated the predictive value of $\mathrm{PNI}$ on biopsy with $\mathrm{PNI}$ on radical prostatectomy as well as the ability of PNI on prostate biopsy to predict adverse findings at radical prostatectomy.

Results: Perineural invasion was present in 124 (26\%) of biopsy specimens diagnosed with prostate cancer and $38(27 \%)$ of those who chose radical prostatectomy. Perineural invasion on prostate needle biopsy was not predictive of radical prostatectomy Gleason score $(p=0.377)$, pathological stage $(p=0.852)$, extraprostatic extension $(p=0.258)$, surgical margin $(p=0.079)$, lymphovascular invasion $(p=0.499)$, and upgrading $(p=0.514)$ or downgrading $(p=0.208)$ at radical prostatectomy. The sensitivity, specificity, positive predictive value, and negative predictive value of PNI on biopsy for PNI on radical prostatectomy were $32 \%, 82 \%, 79 \%$, and $37 \%$ respectively. The Cohen's Kappa correlation coefficient was 0.11 .

Conclusions: Perineural invasion on prostate needle biopsy is not predictive of radical prostatectomy outcome. Furthermore, perineural invasion on biopsy has limited predictive value for perineural invasion at radical prostatectomy.

\section{UP-025}

The Association between Male Pattern Baldness and Second to Forth Finger Ratio with Prostate Cancer: A Prospective Cohort Study

Margel, David; Venkateswaran, Seetha; Darwish, Abbas; Chadwick, Karen; Fleshner, Neil

Division of Urology, Department of Surgical Oncology, University Health Network, University of Toronto, Toronto, ON, Canada

Introduction and Objectives: Retrospective case control studies have demonstrated an association between male pattern baldness and 2D:4D ratio and prostate cancer. The aim of this study was to validate these findings in a prospective cohort.

Methods: Upon approval from our ethical review board we prospectively enrolled 196 consecutive patients referred to a prostate biopsy. Finger lengths were measured using a digital vernier calliper, and the 2D:4D ratio was calculated. Male pattern baldness was assessed on a scale of $0-4$ using the standardized Norwood classification $(0=$ no balding, $1=$ frontal balding, $2=$ Mild vortex, $3=$ moderate vortex and $4=$ severe vortex). We performed all measurements prior to the biopsy thus blinded to the pathology outcome. We used Univariable and multivariable analysis to associate 2D:4D ratio and male pattern baldness with prostate cancer. The multivariable model included the two main predictors (male pattern baldness and 2D:4D ratio) as well age, digital rectal examination and PSA.
Results: The median (IQR) age and PSA of our cohort was 64 (59-70) and 5.8 (4.1-8.4), respectively. Overall 109 patients (55\%) were diagnosed with prostate cancer. On univariable analysis male pattern baldness was associated with prostate cancer ( $p$ for trend $=0.03$ ). However 2D:4D ratio was not. On multivariable analysis male pattern baldness remained a significant predictor of prostate cancer. Furthermore, we noted a dose response effect- the more severe balding patterns were more strongly associated with prostate cancer (Frontal balding OR $2.0(95 \% \mathrm{Cl} 1.1-6.6)$; mild vortex OR 2.1 (95\% $1.5-5.2)$; moderate vortex OR $2.5(95 \% \mathrm{Cl}$ 1.2-7.1); severe vortex OR $2.9(95 \% \mathrm{Cl} 1.1-4.3)$

Conclusions: In a prospective cohort we found that male pattern baldness was an independent predictor of prostate cancer. Further studies are needed in order to assess whether the inclusion of male pattern baldness can contribute to existing models to predict prostate cancer prior to biopsy.

\section{UP-026}

Coexisting Prostate Cancer Found at the Time of Holmium Laser Enucleation of the Prostate for Benign Prostatic Hyperplasia: Predicting Its Presence and Grade in Analyzed Tissue Bhojani, Naeem; Boris, Ronald S.; Mandeville, Jessica A.; Lingeman, James $\mathrm{E}$.

Indiana School of Medicine, Department of Urology, Indianapolis, IN, United States

Introduction and Objectives: Since 1996, HoLEP has been a wellaccepted surgical option for benign prostatic hyperplasia, mimicking anatomic results of open prostatectomy with removal of the entire transition zone. A portion of these men will harbor prostate cancer (PCa) in their analyzed tissue and may go on to subsequent therapy. Establishing risk factors for PCa at the time of HoLEP may aid in preoperative patient counseling. Our study identifies patients with PCa in a large cohort of men undergoing HoLEP and attempts to identify usable variables to predict either PCa or Gleason score at the time of surgery.

Methods: We performed a retrospective data analysis of HoLEP patients at a single institution between 1998 and 2011. At the discretion of the referring urologist, patients with elevated PSAs and/or abnormal digital rectal exams had prior negative preoperative biopsies. Different preoperative and postoperative variables were examined using univariate and multivariate logistic and linear regression models.

Results: Overall, of 1226 HoLEP patients, 109 (8.9\%) had PCa found on tissue analysis (Table 1 ). Almost $3 / 4$ of diagnosed PCa was low grade ( $\leq$ Gleason 6). After univariate and multivariate analysis, only age and weight of specimen were found to predict the presence of PCa (Table 2). More specifically, the risk of PCa increased with increasing age and decreasing gland size. Only rising PSA was predictive of higher Gleason scores on mulitivariate analysis at the time of HoLEP (Table 3).

\begin{tabular}{|c|c|c|c|}
\hline & Benign & Malignant & \\
\hline $\mathrm{N}$ & 1117 & 109 & \\
\hline Mean age & 70.1 & 75.0 & $p<0.001$ \\
\hline Mean PSA & 7.2 & 9.0 & $p=0.08$ \\
\hline $\begin{array}{l}\text { Mean preoperative TRUS } \\
\text { volume }\end{array}$ & 102.3 & 85.8 & $p=0.01$ \\
\hline Mean specimen weight & 80.5 & 64.2 & $p=0.04$ \\
\hline \multicolumn{4}{|l|}{ Gleason score* } \\
\hline$\leq 6$ & N/A & $75(72.1 \%)$ & \\
\hline 7 & $\mathrm{~N} / \mathrm{A}$ & $15(14.4 \%)$ & \\
\hline$\leq 8$ & $\mathrm{~N} / \mathrm{A}$ & $14(13.5 \%)$ & \\
\hline
\end{tabular}

*5 missing values for Gleason score. ; TRUS: transrectal ultrasound; PSA: prostatespecific antigen. 


\begin{tabular}{lc}
\hline \multicolumn{2}{l}{ Table 2. UP-026. Univariate and multivariate analyses } \\
predicting presence of cancer \\
\hline
\end{tabular}

Conclusions: The coexistence of PCa found at the time of HoLEP is low and the majority of patients with cancer will have low grade disease. Older patients with smaller glands appear to be at the highest risk of harboring PCa. In this group of patients only preoperative PSA values appears to influence the presence of more aggressive disease.

\section{UP-027}

Prostate Cancer Screening: Attitudes and Practices of Family Physicians in Ontario

Allard, Christopher ${ }^{1}$; Lusis, Janis ${ }^{2}$; Dason, Shawn ${ }^{1}$; Kapoor, Anil ${ }^{1}$

${ }^{1}$ McMaster Institute of Urology, Hamilton, ON, Canada; ${ }^{2}$ Brampton Hospital, Brampton, ON, Canada

Introduction and Objectives: The utility of prostate cancer (PCa) screening is controversial. We sought to determine whether family physicians in

\begin{tabular}{lc}
\hline $\begin{array}{l}\text { Table 3. UP-026. Univariate and multivariate analysis } \\
\text { predicting Gleason Score }\end{array}$ \\
\hline \\
\cline { 2 - 2 } Univariate & Gleason Score \\
Age & $0.2(p=0.05)$ \\
PSA & $0.29(p=0.04)$ \\
Weight of specimen & $-0.16(p=0.1)$ \\
Preoperative TRUS volume & $-0.15(p=0.21)$ \\
History of biopsy & $-0.07(p=0.5)$ \\
Multivariate & \\
Age & $1.49(p=0.14)$ \\
PSA & $0.31(p=0.01)$ \\
Weight of specimen & $-0.36(p=0.14)$ \\
Preoperative TRUS volume & $0.17(p=0.47)$ \\
History of biopsy & $-0.01(p=0.9)$ \\
\hline TRUS: transrectal ultrasound; PSA: prostate-specific antigen.
\end{tabular}

Ontario, Canada, believe PCa screening is beneficial and to characterize their screening protocols.

Methods: A survey was developed with input from urologists, family physicians, and the Ontario Medical Association's Section on General and Family Practice. Questions covered three domains: demographics, beliefs about screening utility and screening practices. All 7,302 family physicians in Ontario were invited by email to complete the online survey. Results: A total of 969 physicians completed the survey; $955(52.0 \%$ male, $48.0 \%$ female) were included. Most (78.9\%) use PSA and DRE for screening; $9.1 \%$ use DRE alone and $7.0 \%$ PSA; $2.4 \%$ incorporate transrectal ultrasound. $8.3 \%$ do not offer PCa screening. Most physicians begin offering screening at age $50(72.9 \%)$ and stop at ages 70 or $80(68.4 \%)$; $4.3 \%$ offer screening up to age 90 and $17.9 \%$ offer lifelong screening. $54 \%$ offer the same amount of screening as they did 5 years ago, while

\section{Attitudes towards prostate cancer screening}

\section{Strongly Disagree}

Prostate cancer screening should be routinely recommended to men beginning at age 50

The benefits of prostate cancer screening outweigh the risks

Screening with PSA provides a survival benefit

Screening with DRE provides a survival benefit
Neutral

Agree

Strongly Agree

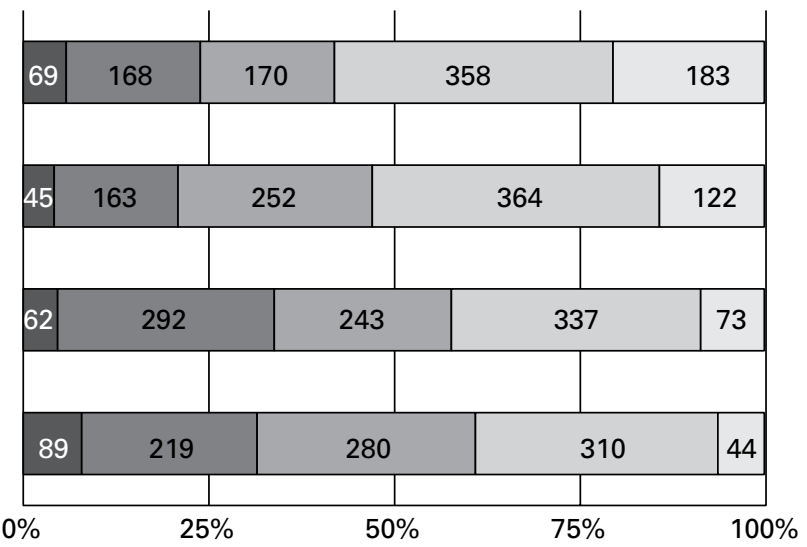

Responses in each category

Fig. 1. UP-027. PSA: prostate-specific antigen; DRE: digital rectal examination. 
$19.5 \%$ offer more and $13.8 \%$ less. Physician beliefs about the utility of PCa screening are shown in Fig. 1.

Conclusions: Although $91.3 \%$ of respondents offer PCa screening, they are divided over its utility, with only $51.4 \%$ convinced that the benefits outweigh the harms. The publications in 2009 of two large randomized controlled trials had a negligible impact on the amount of screening performed by respondents. There is significant variability between physicians' screening beliefs and protocols. A limitation of this study is the possibility of selection bias. Nevertheless, this is the largest sample of Ontario family physicians ever surveyed about PCa screening and highlights divergent physician practices and a need for more conclusive evidence on the subject of screening utility.

\section{UP-028}

\section{Transrectal Ultrasound with Vibroelastography for the Detection} of Prostate Cancer

Gagnon, Louis-Olivier ${ }^{1}$; Mahdavi, Sara ${ }^{2}$; Moradi, Mehdi²; Baghani, $\mathrm{Ali}^{2}$; Jones, Edward ${ }^{3}$; Salcudean, Septimiu²; Goldenberg, Larry ${ }^{1}$

${ }^{1}$ Department of Urologic Sciences, University of British Columbia, Vancouver, BC, Canada; ${ }^{2}$ Electrical and Computer Engineering, University of British Columbia, Vancouver, BC, Canada; ${ }^{3}$ Department of Pathology and Laboratory Medicine, University of British Columbia, Vancouver, BC, Canada

Introduction and Objectives: Vibro-elastography $(\mathrm{VE})$ is a promising technique for imaging soft tissues and relies upon measuring tissue strain in response to a mechanical excitation. The aim of this study is to evaluate the feasibility of detecting cancer within the prostate from VE images.

Methods: Transrectal ultrasound with VE was performed intra-operatively, prior to the prostatectomy, on patients diagnosed with prostate cancer Transfer function images of the prostate, showing the relative stiffness of the tissue within and surrounding the prostate were created. For each case, 9-13 pathology slides extracted from the prostate at approximately 4-mm intervals, with cancer marked, were available. Areas suspected for cancer were marked on the VE images and then compared to the pathology results.

Results: These are preliminary results on 5 patients. Analysis is pending on 5 other patients and recruitment will continue in the coming months. Gleason scores for 51 cancerous areas were available. Twenty of the 31 tumors with Gleason scores of $3+3(64.5 \%), 13$ of the 16 tumors with Gleason scores of $3+4(81.25 \%)$, both tumors with Gleason scores of $4+3$ with tertiary $5(100 \%)$ and both tumors with Gleason scores of $4+5$ $(100 \%)$ were detected. For example, in Figure 1, a tumor of Gleason score $3+4$ is identified. The tumor is $12 \mathrm{~mm}$ by $6 \mathrm{~mm}$ and has an area of $60 \mathrm{~mm} 2$. Overall, VE had a sensitivity of $72.5 \%$ for detecting prostate cancer, with a false negative and a false positive percentage of $24.4 \%$ and $37.3 \%$ respectively. The sensitivity of VE for detecting cancer increased as the Gleason score increased, with a sensitivity of $85 \%$ for tumors with a Gleason score of 7 and above.

Conclusions: This study shows that the use of additional information from VE has the potential of improving the detection of prostate cancer, especially for cancers of higher grade. This imaging method could aid prostate biopsy by highlighting areas suspicious for cancer, reducing the need for repeated biopsy procedures.

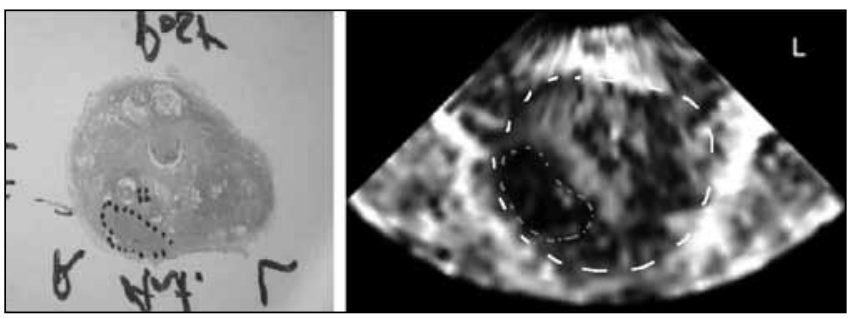

Fig. 1. UP-028. PSA: prostate-specific antigen; DRE: digital rectal examination.

\section{UP-029}

Human Prostate Cancer Magnetic Resonance Elastography and Correlation with Histology

Gagnon, Louis-Olivier ${ }^{1}$; Sahebjavaher, Ramin²; Garteiser, Philippe ${ }^{3}$; Sinkus, Ralph ${ }^{3}$; Baghani, Ali²; Moradi, Mehdi²; Jones, Edward ${ }^{4}$; Nguan, Christopher G. ${ }^{1}$; Goldenberg, Larry ${ }^{1}$; Salcudean, Septimiu ${ }^{2}$

'Department of Urologic Sciences, University of British Columbia, Vancouver, BC, Canada; ${ }^{2}$ Electrical and Computer Engineering, University of British Columbia, Vancouver, BC, Canada; ${ }^{3}$ Hôpital Beaujon, Centre de Recherche Biomédicale Bichat Beaujon (CRB3), Paris, France; ${ }^{4}$ Department of Pathology and Laboratory Medicine, University of British Columbia, Vancouver, BC, Canada

Introduction and Objectives: The aim of this study is to use magnetic resonance elastography (MRE) methods to identify cancerous tumors of the prostate and correlate them to the whole mount histopathology marked with the Gleason score.

Methods: Ethics board approval and informed consent was obtained from a patient (first in study of $\mathrm{N}=20$ ) of age 61 scheduled for radical prostatectomy. The experiments were performed on a 3T Achieva scanner. The vibrations were applied to the perineum using a custom made electromagnetic driver. For the anatomy images, a standard axial T2 weighted FSE sequence was performed. The MRE images were acquired in the axial plane using a novel fast field echo sequence named eXpresso. The wave images were acquired on a matrix with $2 \mathrm{~mm}$ isotropic voxel size. Eight vibration phases were encoded at a mechanical excitation of $70 \mathrm{~Hz}$.

Results: The peak amplitude of the mechanical wave was $130 \mu \mathrm{m}$ with a mean of $25 \mu \mathrm{m}$ in the prostate. No patient discomfort was reported when specifically asked. In the axial plane, the prostate gland is outlined in the T2W (a), and the reconstructed shear modulus $\mathrm{G}^{\prime}(\mathrm{b})$ and loss modulus $\mathrm{G}^{\prime \prime}$ (c). The histology is shown in (d) where the outline of the large (Gleason score of $4+3)$ and smaller $(3+3)$ tumors are shown. A very promising correspondence between reconstructed shear and loss moduli $G^{\prime}$ and $G^{\prime \prime}$ and the matching histology slide can be observed. The mean values of $\mathrm{G}^{\prime}$ were $3.0,1.6$, and $0.8 \mathrm{kPa}$ for Gleason scores of $4+3,3+3$, and healthy tissue, respectively. Also, the mean values for $\mathrm{G}^{\prime \prime}$ were $1.7,0.8$, and 0.4 $\mathrm{kPa}$ for the same regions of interest.

Conclusions: This is, to the best of our knowledge, the first in-vivo prostate cancer patient MRE images that are correlated with whole mount histology. These early results confirm that cancerous tissue in prostate specimens has higher stiffness and also higher viscosity compared to healthy tissue. MRE is a promising tool in order to improve diagnosis and staging of prostate cancer tumors.

\section{UP-030}

Changes in Positive Surgical Margins over Time Reflect a Risk Migration in Patients Undergoing Radical Prostatecomy at a Tertiary Care Centre

Longpre, Michelle; Gleave, Martin; Harriman, David; Hurtado-Coll, Antonio

University of British Columbia, Vancouver, BC, Canada

Introduction and Objectives: Positive surgical margin (PSM) rates after radical prostatectomy (RP) have been shown to be as low as $4 \%$ and as high as $48 \%$ in high volume centres. The incidence of PSM varies by pathological stage but can also be influenced by surgical techniques and by pathologist interpretation. PSM are predictive of biochemical recurrence but a significant proportion will not recur. In this study we sought to determine changes in incidence of PSM rates over time as lower risk patients opt for active surveillance and whether this was related to a shift in pathological stage and grade.

Methods: PSM, clinical and pathological staging, as well as PSA recurrence in almost 2,000 patients who underwent RP from 1993 to present was extracted from a prospectively recorded prostate cancer database at Vancouver General Hospital, BC, Canada. Patients were grouped by their date of surgery into either remote $(1993-2004)$ or recent (20052011) cohorts. All pathological pT2 and greater patients were included. Regression models were developed to predict the rate PSM with adjustment for known confounders. 
Results: Remote $(765)$ and recent $(1,216)$ radical prostatectomies were compared. The PSM rate was $21.7 \%$ from the remote cohort and $27 \%$ from the recent cohort $(p=0.027)$. The postoperative Gleason grades shifted significantly from $45.97 \%$ Gleason $6,43.83 \%$ Gleason 7 , and $10.20 \%$ Gleason greater than 8 in the remote cases to $17.4 \%, 67.46 \%$ and $15.30 \%$ respectively in the recent cases $(p \leq 0.0005)$. The percentage of patients with clinically high-risk disease was also significantly greater in the recent cohort $(p \leq 0.0005)$.

Conclusions: The PSM rates in our tertiary care high volume centre are similar to other reported centres. The increase in PSM rates is likely due to a shift in patient population from lower risk patients to higher risk patients as lower risk patients opt for active surveillance.

\section{UP-031}

Holmium Laser Ablation of the Prostate Gland (HoLAP) Followed by Brachytherapy (BT) for Treatment of Patients with Clinically Localized Prostate Carcinoma (PC) and Obstructive Urinary Symptoms (LUTS)

Kumar, Surendra

Oakwood Annapolis Hospital, Wayne, MI, United States

Introduction and Objectives: Patients with localized prostate carcinoma and LUTS who undergo BT with permanent seed implants have a 40 to $50 \%$ chance of going into urinary retention or requiring a catheter for intermittent self-catheterization (ISC) due to worsening of their obstructive urinary symptoms. The purpose of this study was to assess the role of HoLAP prior to BT in preventing post BT urinary complications.

Methods: 86 patients age 50-83 (mean 67.6) years with LUTS and clinically localized prostate carcinoma underwent HoLAP with a $100 \mathrm{~W}$ holmium laser under spinal anesthesia. The end point of the procedure involved complete vaporization of obstructing prostate tissue down to the capsular fibers and appearance of an open prostate cavity. Patients underwent BT 7-45 (mean 16) weeks after HoLAP when they had recovered from the procedure. BT was done by real-time interactive ultrasoundguided (lodine-125 or Palladium-103) seed placement with peripheral loading under general anesthesia.

Results: HoLAP significantly reduced prostate volume an average $25 \mathrm{cc}$ $(44 \%)$, reduced the mean AUA symptom score by 13 points $(84 \%)$ and increased mean Q-max by $11 \mathrm{cc} / \mathrm{sec}(121 \%)$ (Table 1). There was no clinically significant change in AUA symptom score or Q-max following BT with a follow-up of 0.43-6.91 (mean 3.65) years (Table 2). No patient experienced prolonged urinary retention after BT and none has required ISC. No patient developed stress incontinence after HoLAP and BT. Conclusions: Patients with prostate carcinoma and LUTS who have undergone HoLAP to relieve their obstructive urinary symptoms prior to BT

\begin{tabular}{|c|c|c|c|c|c|}
\hline Variable & $\mathbf{n}$ & Pre-Hol & Post-Hol & $\Delta \%$ & $p$ \\
\hline Prostate Vol. & 86 & $57.23 \pm 24.22$ & $32.19 \pm 13.01$ & -44 & $<0.001$ \\
\hline AUA Score & 86 & $15.93 \pm 4.60$ & $2.57 \pm 1.27$ & -84 & $<0.001$ \\
\hline Q-max & 76 & $9.63 \pm 3.02$ & $20.43 \pm 10.28$ & +121 & $<0.001$ \\
\hline $\mathrm{Na}$ & 86 & $138.72 \pm 2.02$ & $137.95 \pm 2.27$ & -0.5 & 0.001 \\
\hline $\mathrm{Hb}$ & 86 & $14.36 \pm 1.34$ & $13.60 \pm 1.40$ & -5 & $<0.001$ \\
\hline
\end{tabular}

$\Delta \%$ Change $=($ Post-Holap - Pre-Holap $) /($ Pre-Holap $) * 100$.

Table 2. UP-031.Clinical parameter changes after HoLAP and BT

\begin{tabular}{llcc}
\hline & N & Post HoLAP & Post BT \\
\hline AUA Score & 86 & 2.57 & 1.71 \\
Omax (cc/sec) & 71 & 20.71 & 19.68 \\
\hline
\end{tabular}

Mean duration of follow-up after BT 3.65 yrs. BT: Brachytherapy. do not experience prolonged urinary retention, worsening of their LUTS or stress incontinence following real-time interactive ultrasound-guided radiation seed placement with peripheral loading. With elimination of obstructive urinary symptoms and an approximately $44 \%$ reduction in the prostate volume by HoLAP a larger pool of patients with prostate carcinoma can benefit from modern brachytherapy.

\section{UP-032}

Identification of Thrombotic Risk for Men with Advanced Prostate Cancer: a Pilot Study Evaluating Hemostatic Status Using Thromboelastography

Siemens, D. Robert; Toukh, Mazen; Othman, Maha; Black, Angela; Graham, Charles

Queen's University, Kingston, ON, Canada

Introduction and Objectives: Coagulopathy is the second most common cause of death from cancer, and thrombotic complications are amplified in prostate cancer with systemic therapy. We aim to help identify patients at higher risk for thrombotic events in patients with prostate cancer with well-defined hemostatic tests, novel in their application to patients with advanced prostate cancer.

Methods: We performed intensive haemostatic studies in 27 patients (age range $59-88$ years) at various stages (non-metastatic, metastatic, castration resistant) as compared to an age-matched control group (biopsy negative, $\mathrm{n}=9$ ). Thromboelastography (TEG) is a global haemeostatic test that quantifies a vesicoelastic trace that reflects the kinetics of clotting. The study included whole blood TEG and flow cytometry analysis of microprticles (MPs) in plasma using Annexin V- FITC and anti-tissue factor - PE.

Results: Analysis of the data revealed hypercoagulable state in all patients with advanced disease. The mean values for TEG parameters in the patients were: R: 6.01 vs. 9.8 minutes in the control group ( $p=0.009)$, alpha angle: 68.3 (controls 53.1 degrees), MA: 69.3 vs. $57.9 \mathrm{~mm}$ in controls ( $p=0.053$ ), and $\mathrm{Cl}: 3.32$ vs. 0.7 in controls $(p=0.05)$. Microparticle assays revealed significant elevation in the number of microparticles carrying tissue factor in these patient groups compared to the control group [5,142 MPs/uL compared to 2,914 MPs/ uL ( $p=0.05)$ ], suggesting a link between elevated tissue factor and the hypercoagulability.

Conclusions: To our knowledge, this is the first report for the use of TEG in patients with advanced prostate cancer. These results would suggest the rationale for a larger cohort study to determine the utility of these simple tools for evaluation of patients' thrombotic potential and may help identification of those who require anticoagulant prophylaxis towards the end of their cancer journey.

\section{UP-033}

Predictors of Failing Active Surveillance on the 2nd Prostatic Biopsy

Wong, Lih-Ming'; Trottier, Greg'; Fleshner, Neil'; Lawrentchuk, Nathan ${ }^{1}$; Kulkarni, Girish'; Zlotta, Alexandre R. ${ }^{2}$; Trachtenberg, John'; Toi, Ants ${ }^{3}$; Finelli, Antonio

${ }^{1}$ Division of Uro-oncology, Princess Margaret Hospital, Toronto, ON, Canada; ${ }^{2}$ Division of Urology, Mount Sinai Hospital, Toronto, ON, Canada; ${ }^{3}$ Department of Radiology, Princess Margaret Hospital, Toronto, ON, Canada

Introduction and Objectives: Active surveillance (AS) is a popular method to minimize the morbidity associated with overtreatment of prostate cancer. The rate of patients who progress by grade/volume is reported as $9-35 \%(1)$. We examine factors from the 2 nd biopsy, whilst on AS, that may predict pathological progression and need for subsequent therapy. Methods: We identified patients from our prospectively maintained, single academic institution, database with PSA $<20$, Gleason sum (GS) $\leq 6$, stage T1 c, $\leq 3$ cores positive for cancer, $<50 \%$ of single core involved, age $\leq 75 \mathrm{y}$ years and had a repeat biopsy within 48 months after the initial biopsy $(n=312)$. Logistical regression was performed on available data $(n=278)$ to identify predictors of pathological progression, which was defined as GS $\geq 7,>3$ positive cores and $>50 \%$ tumor involvement of any single core. Results: Of the 278 patients included, 48 patients had pathological pro- 
gression on 2nd biopsy and 264 did not. For both groups, median number of biopsy cores taken at 2 nd biopsy was the same $(n=15)$. Predictors of pathological progression found were PSA velocity (for every 1 unit increase, OR $1.12(1.03-1.21), p=0.01)$, total number of cores taken at first biopsy <10 (OR $2.78(1.2-6.67), p=0.02)$, and time between 1 st and 2 nd biopsies (for every 1 month increase, OR 1.05 (1.02-1.08), $p=0.002$ ). Conclusions: We have identified predictors of progression for patients on AS. Patients who had $<10$ cores on initial biopsy should have confirmatory biopsy to avoid under-sampling. Delay in 2 nd biopsy should be avoided and rapid PSAV should trigger early re-biopsy.

\section{UP-034}

\section{Prostate Cancer Detection after Multiple Negative Biopsies}

Metcalfe, Charles; Metcalfe, Michael; Goldenberg, Larry; Gleave, Martin Black, Peter; So, Alan

University of British Columbia, Vancouver, BC, Canada

Introduction and Objectives: The rates of false negative prostate biopsies range between $10-20 \%$. There is limited evidence describing the prognosis of prostate cancers (PCa) diagnosed after previous negative biopsies. We evaluate the indicators for multiple repeat biopsies, predictive factors of tumors that are eventually diagnosed, and clinical parameters that differentiate patients that are later diagnosed with clinically significant disease from low risk cancers after initial negative biopsy.

Methods: A retrospective search was performed over 20 years of patients with a new diagnosis of $\mathrm{PCa}$ and at least two prior negative prostatic biopsies. Predictive clinical and pathologic data were examined prior to eventual positive biopsy and associated with grade and management decisions/outcomes.

Results: A total of 70 patients with 2 to 7 prior negative prostatic biopsies were included. 20 patients had a Gleason Score (GS) $\geq 8,30$ GS 7, 20 were GS $<7$. A persistently elevated or rising PSA and suspicious pathology on prior biopsy were the two main indications for repeat biopsies. Prior atypical acinar small proliferation (ASAP) and/or high-grade prostatic intraepithelial neoplasia (HGPIN) were significant risk factors for later PCa diagnosis. DRE, PSA and PSA velocity were not significant in cancer diagnosis. $67 \%$ of patients underwent radical prostatectomy, $23 \%$ on active surveillance, $4 \%$ had radiation and $3 \%$ systemic hormonal therapy. The number of previous biopsies did not predict for either tumor volume of GS at diagnosis.

Conclusions: Persistently rising PSA as well as ASAP and HGPIN predict for future positive diagnosis of PCa. A high number of intermediate and high-risk prostate cancers were diagnosed in those with prior negative prostate biopsies. Most importantly, the number of prostate biopsies did not predict for smaller low-grade cancers and point to the importance of vigilance in patients suspected of having prostate cancer with a persistently rising PSA.

\section{UP-035}

Intermediate Clinical Outcomes of Robot-assisted Laparoscopic Prostatectomy (RALP)

Wong, Carson'; $\mathrm{Gu}, \mathrm{XiaO}^{2}$; Spaliviero, Massimiliano ${ }^{2}$

${ }^{1}$ SouthWest Urology, Inc., Middleburg Heights, $\mathrm{OH}$, United States; ${ }^{2}$ University of Oklahoma Health Sciences Center, Oklahoma, OK, United States

Introduction and Objectives: We review our experience of RALP with a minimum follow-up duration of 24 months. The hospital records of consecutive patients who underwent transperitoneal RALP by a single surgeon $(\mathrm{CW})$ were reviewed.

Methods: A bladder neck sparing dissection was preferentially performed and the urethrovesical anastomosis was completed using a running double-armed 3-0 Monocryl suture. On postoperative day 5 or 6 (clinic logistics), the urethral catheter was removed following a normal cystography. Results: Clinical outcomes and adverse events are presented. 233 patients had a mean age of $62.7 \pm 6.7$ years and serum PSA of $6.2 \pm 4.6 \mathrm{ng} / \mathrm{mL}$. Median operative time was 190 minutes and estimated blood loss was $75 \mathrm{~mL} .3(1.3 \%)$ patients required bladder neck reconstruction, while 198 $(85.0 \%)$ had bilateral, $20(8.6 \%)$ had unilateral and $15(6.4 \%)$ did not undergo nerve sparing prostatectomy. 199 (85.4\%) patients had negative surgical margins. Median hospitalization and urethral catheter duration were 1.0 and 5.0 days, respectively. At 6 weeks, a median 1.0 pad per day usage was reported. $69.1 \%$ of patients achieved urinary continence without pads at the 3 month follow-up interval and $95.7 \%$ of patients were continent at 12 months. $52.3 \%$ of patients having a nerve sparing procedure achieved potency within 24 months following RALP. The incidence of adverse events were low: 5 (2.1\%) patients had prolonged urine leak, $3(1.3 \%)$ patients experienced a pelvic hematoma, 1 one $(0.4 \%)$ patient had a urinary tract infection, and $2(0.9 \%)$ and $5(2.1 \%)$ patients developed deep vein thrombosis and bladder neck contractures (BNC), respectively. $95.3 \%$ and $96.5 \%$ of patients at 12 and 24 months, respectively, had an undetectable serum PSA $(<0.2 \mathrm{ng} / \mathrm{mL})$. Five patients had adjuvant radiotherapy for positive surgical margins or PSA recurrence.

Conclusions: RALP is an effective treatment option for clinically localized prostate cancer that preserves ones quality of life with low patient morbidity.

\section{UP-036}

Factors Influencing Choice of Robotic-assisted vs. Open Radical Prostatectomy for Prostate Cancer Treatment

Chien, Gary; Chang, Allen; Thomas, Anil; Derboghossians, Armen; Slezak, Jeffrey

Kaiser Permanente, Los Angeles, CA, United States

Introduction and Objectives: Despite its debated benefits, much of the rapid adoption of robotic-assisted laparoscopic prostatectomy (RALP) in USA has been attributed to aggressive advertising, market demand, and hospital utilization pressures. In our study, we aim to examine RALP vs. radical retropubic prostatectomy (RRP) utilization rates in a large health maintenance organization where marketing and financial incentives are minimized.

Methods: Patients were grouped to either RRP or RALP from a prospectively collected prostate cancer database at Kaiser Permanente Southern California (KPSC). Demographics, clinicopathologic data, and surgeon profiles were recorded. Descriptive statistics and chi-squared analysis were used.

Results: From March to October 2011, 500 patients undergoing RRP vs. RALP were identified. The majority, 455 (91\%) patients underwent RALP and $43(9 \%)$ underwent RRP, with a median age of $60(37-77)$ and 62 (39-70), respectively. There were a total of 21 RALP surgeons and 17 RRP surgeons, with 2 surgeons in both groups. The mean age of RALP surgeon was 40 years while the mean age of RRP surgeon was 49 years $(p=0.0007)$. Overall, $13(76 \%)$ RRP surgeons were out of training $>10$ years compared to $3(14 \%)$ of RALP surgeons. The majority of patients was clinical stage T1c ( $71 \%$ for RALP group and $84 \%$ for RRP group) and had a biopsy Gleason score of 6 (53\% for RALP group and $60 \%$ for RRP group). There was no statistically significant difference in patient age $(p=0.5)$, race $(p=0.61)$, clinical stage $(p=0.78)$, biopsy Gleason score $(p=0.91)$ or final pathologic stage $(p=0.32)$ between the two groups.

Conclusions: Within our health maintenance organization, RALP remains the most common modality of surgery for prostate cancer. The surgeon's age and years out of training may influence the decision to perform RRP, rather than preoperative patient clinical characteristics.

\section{UP-037}

What Is the Prevalence and Impact of Depression, Anxiety, and Distress in Patients with Newly Diagnosed Localized Prostate Cancer?

Punnen, Sanoj; Cowan, Janet; Whitson, Jared; Carroll, Peter; Cooperberg, Matthew

University of California, San Francisco, CA, United States

Introduction and Objectives: Mental health concerns and their impact on recovery often go unnoticed in men with prostate cancer. The study objective was to determine the prevalence of depression, anxiety and distress over time among active surveillance and surgical patients and to investigate the affect these mental health measures may have on urinary and sexual quality of life over time.

Methods: The study population consisted of patients who were managed 
with active surveillance (AS) or radical prostatectomy (RP). Baseline levels of depression, anxiety and distress were ascertained using well-validated questionnaires: Patient Health Questionnaire 9(PHQ-9), Generalized Anxiety Disorder 7 (GAD-7) and the Distress Thermometer (DT), respectively. Multivariate logistic regression examined associations between clinical factors and mental health while mixed model repeated measures analysis examined the affect of baseline depression, anxiety and distress on sexual and urinary quality of life in follow-up.

Results: Among the 907 men that made up the study cohort moderate or higher levels of depression or anxiety appeared low $(<5 \%)$ while levels of mild depression or anxiety ranged from $7-15 \%$. Levels of high distress ranged from $6-18 \%$. There appeared to be no difference between active surveillance and surgery patients except at 1-3 years, where active surveillance patients showed more anxiety. Worse urinary and sexual function, decreased age and not being in a relationship were associated with worse mental health. Increased levels of depression, anxiety or distress at baseline were associated with worse urinary and sexual function and bother in follow-up.

Conclusions: Levels of moderate or higher depression, anxiety and distress may appear low at baseline and follow-up, however, worsening mental health at baseline was associated with worse urinary and sexual outcomes over time.

\section{UP-038}

Autologous Retropubic Urethral Sling: a Novel, Quick, Intraoperative Technique for Improving Continence after Robotassisted Laparoscopic Prostatectomy

Punnen, Sanoj; Cowan, Janet; Whitson, Jared; Carroll, Peter

University of California, San Francisco, CA, United States

Introduction and Objectives: We describe a novel technique using the medial umbilical ligament or vas deferens as an autologous retropubic urethral sling placed at the time of robotic assisted laparoscopic prostatectomy (RALP) and evaluate its impact on postoperative continence. Methods: In 2011, men who underwent sling placement were compared to men who did not have a sling placed. Slings were placed primarily in men who were expected to have worse recovery of urinary continence. Sling placement involved harvesting a segment of the medial umbilical ligament or vas deferens, placing it under the vesico-urethral anastomosis, affixing it to the pubic symphysis, and adjusting the tension to create a slight elevation of the vesicourethral angle. The association of sling placement on time to one and no pads per day (PPD) was assessed using Cox proportional hazards regression analysis.

Results: The study cohort consisted of 54 men who underwent sling placement and 41 men who did not during the same time period. Median follow-up was 4.8 months in the sling patients and 5.5 months in the non-sling patients. Clinical and pathological characteristics were similar between the groups, with the exception of sling patients displaying more high-grade disease and less nerve sparing $(p<0.01)$. There was no significant association seen between placement of a sling and time to 1 PPD on either univariate or multivariate analysis. There was trend towards a benefit of sling placement in time to 0 PPD on univariate analysis $(p=0.08)$, but this failed to reach statistical significance and was attenuated after adjustment for demographic and clinical covariates.

Conclusions: Although the association between sling placement and early continence did not reach statistical significance, there was a trend towards a benefit in sling patients in time to no PPD. Randomized trials are needed to assess the true benefit of sling placement on continence outcomes.

\section{UP-039 \\ Predictors of Gleason 8 Cancer among Men with Gleason 7 and 8 Cancer on Prostate Biopsy Cores}

Heimrath, Olivier P.; Belanger, Eric C.; Kos, Zuzana; Cagiannos, Ilias; Morash, Christopher G.; Lavallée, Luke T.; Preston, Mark A.; Breau, Rodney $\mathrm{H}$.

University of Ottawa, Ottawa, ON, Canada

Introduction and Objectives: The purpose of this study was to determine the incidence of intermediate and high-risk disease among men with discordant prostate biopsy grades.

Methods: Consecutive patients with at least one prostate biopsy core of Gleason 8-10 cancer AND at least one prostate biopsy core of Gleason $<8$ cancer treated with radical prostatectomy (RP) at The Ottawa Hospital between 1995 and 2010 were reviewed. Candidate predictors were the proportion of Gleason 3 cancer from all biopsy tissue, the proportion of biopsy cores that contain Gleason 3 cancer, the number of cores that contained Gleason 3 cancer and the highest proportion of Gleason 3 cancer on any core. The primary outcome was final prostate cancer Gleason score from the RP specimen.

Results: Of 750 radical prostatectomies, 22 (3\%) met inclusion criteria. Of these, $11(50 \%)$ had a final Gleason sum of 7 or less, while $11(50 \%)$ had a final Gleason 8 or more. Of the 7 men with at least one core Gleason 9 , only $1(14 \%)$ had a final Gleason sum less than 8 . The proportion of biopsy tissue that was Gleason 3 was $5.0 \%$ (SD $4.9 \%$ ) versus $6.3 \%$ (SD $8.4 \%$ ) for patients with RP Gleason score of less than 8 compared to 8 or more $(p=0.97)$. The respective, mean proportion of cores that had Gleason 3 was $31.4 \%$ (SD 21.8\%) compared to $35.1 \%$ (SD 31.3\%) and the median number of cores that had Gleason 3 was 3 (IQR 2-4) compared to 2 (IQR $1-4)$. On average the highest proportion of Gleason 3 in any core also did not predict RP Gleason grade with $26.4 \%$ (SD 23.9\%) compared to $24.6 \%$ (SD 16.2\%) $(p=0.95)$.

Conclusions: When a patient has a biopsy core of 8 or more and a discordant biopsy core of less than 8 , he has a $50 \%$ risk of an RP Gleason score of 8 or more. When one core is Gleason 9 or more, almost all patients have Gleason 8 or more in the RP specimen. The proportion of Gleason 3 cancer in the biopsy specimens was not predictive of RP Gleason grade. Further study to verify our findings using larger samples is warranted.

\section{UP-040}

Comparison of Serum Testosterone Levels in Prostate Cancer Patients Receiving LHRH Agonist Therapy with or without the Removal of the Prostate

Venkateswaran, Seetha'; Margel, David ${ }^{1}$; Yap, Stanley ${ }^{1}$; Hersey, Karen ${ }^{1}$; Locke, Jennifer ${ }^{1}$; Yip, Paul ${ }^{2}$; Fleshner, Neil ${ }^{1}$

${ }^{1}$ Princess Margaret Hospital, Toronto, ON, Canada; ${ }^{2}$ Toronto General Hospital, Toronto, ON, Canada

Introduction and Objectives: The prostate is an endocrine organ whose primary function is secreting enzymes and nutrients promoting sperm motility. Recent reports suggest that the prostate may also secrete testosterone, believed to be a fuel for prostate tumor growth. The aim of this study was to determine if a difference in serum testosterone levels exists between men on LHRH agonists who have undergone radical prostatectomy, radiation or hormone therapy as primary prostate cancer treatment. Methods: Serum testosterone levels were evaluated among 165 consecutive PCa patients using LHRH analogues for $>3$ months. We excluded patients receiving either radiation or chemotherapy at time of time of testosterone measurement. Patients were classified based on primary treatment: 1 ) radical prostatectomy $(\mathrm{RP})$; 2 ) radiation $(\mathrm{Rx})$ or 3 ) primary hormone therapy $(\mathrm{PH})$. One-way ANNOVA was used to compare testosterone levels. Pearson correlation was used to correlate testosterone with PSA and time on LHRH agonists. Multivariable linear regression was used to predict serum testosterone levels.

Results: The median (IQR) serum testosterone levels were 1.4 (1-1.9), 1.3 $(1-1.625)$ and $1.25(0.9-1.525) \mathrm{nmol} / \mathrm{L}$ for RP, Rx and PH groups respectively. There was no statistically significant difference in testosterone levels between the groups $(p=0.3)$. No correlation was found between testosterone and PSA levels or time on $\mathrm{LHRH}(\mathrm{R}=0.02$ and $\mathrm{R}=0.01)$ respectively. 
Multivariable linear regression showed that none of the clinical variables were predictors of testosterone levels.

Conclusions: Our study suggests that primary treatment does not affect serum testosterone levels among men using LHRH analogues. Preliminary data also suggests that surgical removal of the prostate may not confer an added advantage in reducing testosterone levels.

\section{UP-041}

A Prospective Phase II Trial of Stereotaxic Hypofractionated Cyberknife (CK) Treatment for Locally Recurrent Prostate Adenocarcinoma Following External Beam Radiotherapy (EBRT) Fortin, Israel; Bahary, Jean-Paul; Saad, Fred; Péloquin, Laurence; Lambert, Carole; Barkati, Maroie; Nguyen, Thu Van; Waters, Alexandra

The Centre hospitalier de I'Université de Montréal, Montreal, QC, Canada Introduction and Objectives: EBRT is frequently used as primary treatment for prostate cancer. Although EBRT can achieve a really good biochemical control at long term, some residual tumor can subsist. Those focci could further de-differentiate, disseminate and cause metastatic disease. Patients presenting a locally recurrent prostate cancer post-EBRT are usually put on a palliative treatment. Curative treatment such as cryotherapy, prostatectomy, brachytherapy and Hi-FU can be considered. However, there is considerable concern with their potential sides effects. Retrospectives studies of post-EBRT hypofractionated treatments with CK have shown promising results. Consequently, we propose to study the feasibility of reirradiation in patients presenting a biochemical relapse post-EBRT and design a Phase I-II trials to evaluate the genitourinary toxicities as well as the local control (LC) and the overall survival (OS).

Methods: We record the doses given for prostate reirradiation in the literature. Prostate MRI perfusion were performed to access the ability to detect locally recurrent prostate carcinoma after previous EBRT. With the selected fractionations, we perform a preliminary study. Simulations on a computerized treatment planning system were done to evaluate the feasibility of the treatment. A Phase I-II protocol was then written on the basis of those findings.

Results: A CK treatment of 25,30 and 35Gy is achievable in 5, 6 and 7 fractions respectively without overpassing organ at risk dose restrictions. Our feasibility study shows that prostate MRI perfusion is a good tool in identifying prostatic relapse focci.

Conclusions: Locally relapsing focci can be identified with a MRI perfusion in patient presenting a recurrent prostate cancer post-EBRT. A CK hypofractionated treatment can potentially be curative. A Phase I-II trial is currently recruiting and will evaluate the associated toxicities as well as the OS and the LC. The results of the first cohort of patients will be presented at the meeting

\section{UP-042}

Involvement of TBK1 in Prostate Cancer Progression through IKK $\varepsilon$ Cellular Localization Control

Huon, Yannick'; Péant, Benjamin'; Mes-Masson, Anne-Marie'; ${ }^{1}$ Saad, Fred $^{2}$ ${ }^{1}$ CRCHUM, ICM and Faculty of Medicine, Université de Montréal, Montreal, QC, Canada; ${ }^{2}$ CRCHUM, ICM, Faculty of Medicine and Division of Urology, CHUM, Université de Montréal, Montreal, QC, Canada

Introduction and Objectives: Overexpression of inflammatory cytokines IL-6 and IL-8 has been associated with hormono-sensitive (HS) prostate cancer (PCa) progression to a castrate-resistant status (CR). In HS cells, cytoplasmic expression of the kinase IKKepsilon (IKKE) is inducible in response to infections. Conversely, in CR cells, IKKE is constitutively expressed in the cytoplasm and the nucleus. To study IKKE cellular regulation in $\mathrm{PCa}$, we have focused on its cytoplasmic partner, TANK-binding kinase 1 (TBK1). Recent studies have shown the requirement of TBK1 in oncogenesis but its function in PCa remains unclear. We hypothesize that TBK1 acts to sequester IKKE to the cytoplasm. However, IKKE overexpression disturbs the stoichiometric balance in CR cell lines, thus permitting its nuclear translocation and IL-6/IL-8 secretion.

Methods: To elucidate these molecular events during PCa progression, we have designed inducible lentiviral constructs that allow us to control
TBK1 or IKKE expression. We generated HS and CR clones, in which TBK1 expression can be knocked-down using inducible shRNA. We also selected constructs that enable overexpression of TBK1 in CR cells. The cellular localization of IKKE is monitored by Western blot analysis and its nuclear activity by cytokine secretion quantified by ELISA.

Results: The investigation of TBK1 knock-down in CR clones did not reveal an increase of IKKE in the nucleus. To confirm this, we are overexpressing TBK1 in CR cells to maintain IKKE in the cytoplasm. We are also evaluating the consequence of TBK1 knock-down in HS clones when IKK $\varepsilon$ expression is induced. Knowing that in this model IKKE remains within the cytoplasm, we expect to observe IKKE nuclear accumulation. Conclusions: Loss of TBK1 in CR cell lines does not support our hypothesis, although in these cells this may be due to initial saturation of IKKE. Further experiments are ongoing to more fully elucidate a possible relationship between TBK1, IKKE and its nuclear translocation.

\section{UP-043}

The Association between Aspirin and Non-steroidal Antiinflammatory Drug Use and the Risk of Prostate Cancer Detection upon Biopsy

Bhindi, Bimal; Margel, David; Fernandes, Kimberly; Trottier, Greg; Hersey, Karen; Finelli, Antonio; Trachtenberg, John; Toi, Ants; Evans, Andrew; van der Kwast, Theodorus H.; Fleshner, Neil

University Health Network, Toronto, ON, Canada

Introduction and Objectives: Chronic inflammation plays an important role in carcinogenesis. Interest has been generated in determining if COX-inhibitors reduce prostate cancer (PCa) risk. Our objective was to study the association between aspirin (ASA) and other non-steroidal antiinflammatory drugs (NSAIDs) and PCa detection using a cohort undergoing prostate biopsy.

Methods: Using a prospectively maintained database of patients undergoing prostate biopsy, ASA and other NSAID use, biopsy results, and other clinical variables were ascertained by questionnaire and chart review. Hypothesis tests were conducted using the Chi-Square and Kruskal Wallis tests for categorical and continuous variables, respectively. Univariable and multivariable analyses were performed to explore the associations between exposure to ASA and other NSAIDS and PCa outcomes.

Results: Of the 931 patients in our cohort, prostate biopsy diagnosed PCa at a significantly higher rate among patients taking ASA $(65 \%, p<0.001)$ and those taking other NSAIDs $(65 \%, p<0.001)$ compared to those taking no NSAIDs $(42 \%)$. Patients taking ASA $(38 \%, p<0.001)$, but not those taking other NSAIDs $(21 \%, p=N S)$, were more likely to be diagnosed with HGPCa compared to those not taking NSAIDs (21\%). On multivariate analysis, ASA use $(\mathrm{OR}=2.16, p=0.0002)$ and other NSAID use (OR=3.00, $p=0.0004$ ) were associated with higher odds of PCa detection, while ASA use was associated with a higher odds of HGPCa detection $(\mathrm{OR}=1.64$, $p=0.038$ )

Conclusions: In contrast to the majority of epidemiological studies in the literature, ASA and other NSAID use in our cohort were associated with a higher risk of detecting PCa, while ASA use was associated with a higher risk of detecting HGPCa. These medications may still have chemopreventative properties, such that patients whose cancers were prevented were never referred for biopsy. However, studies are needed to further assess the impact of our findings on PCa screening risk assessment.

\section{UP-044-WITHDRAWN}

\section{UP-045-WITHDRAWN}




\section{UP-046}

\section{Active Surveillance for Prostate Cancer in Patients with a PSA} $>10$

Toren, Paul; Timilshina, Narhari; Kulkarni, Girish; Fleshner, Neil; Finelli, Antonio

University of Toronto, Toronto, ON, Canada

Introduction and Objectives: The use of prostate specific antigen (PSA) in active surveillance (AS) for prostate cancer is controversial. Several published series use an entry PSA $>10 \mathrm{ng} / \mathrm{mL}$ as an exclusion from $\mathrm{AS}^{1-3}$. Since we do not use an elevated PSA as an absolute contraindication to start or continue AS, we set to review our experience as to the significance of a PSA value over $10 \mathrm{ng} / \mathrm{ml}$ in patients on AS.

Methods: Our cohort included all patients on AS with clinical stage T1c$\mathrm{T} 2 \mathrm{a}$, Gleason score $\leq 6$, and three or fewer cores positive with no more than $50 \%$ of a core involved at initial diagnostic biopsy. Patients with less than two biopsies were excluded. Patients were grouped into those who 1) started AS with a PSA $>10 \mathrm{ng} / \mathrm{mL}, 2$ ) had a PSA rise $>10 \mathrm{ng} / \mathrm{mL}$ during follow-up and 3) had a PSA $<10 \mathrm{ng} / \mathrm{mL}$ throughout AS. Pathologic progression (PP) was defined as biopsy parameters exceeding the entry criteria limits.

Results: There were 340 patients in our cohort according to the biopsy entry criteria with at least 2 biopsies on AS. The whole cohort had a mean age of 64.3 years and a median follow-up of 45.6 months. Initial biopsy characteristics did not differ between groups. Median PSA $(\mathrm{ng} / \mathrm{mL})$ at initial biopsy was $12.3,6.8$ and 4.5 for groups $1-3$, respectively $(p<0.001)$. Median prostate volume $(\mathrm{mL})$ was 61,51 and 42 for groups $1-3$ respectively $(p<0.001)$. Outcomes by group are in Table 1 . PP at first biopsy was not different between groups $(p=0.44)$, nor was the proportion who had treatment $(p=0.36)$. Using logistic regression analysis, PSA density was not predictive of PP at 1st repeat biopsy, even after adjusting for group. Conclusions: With careful patient selection, those with a PSA $>10$ do not necessarily need to be excluded from active surveillance. In our cohort those starting AS with a PSA $>10 \mathrm{ng} / \mathrm{mL}$ did not have a higher rate of pathologic progression at first repeat biopsy compared to those following stricter criteria.

\section{UP-047}

\section{Targeting the Proprotein Convertases in Prostate Cancer}

Day, Robert'; D'Anjou, François ${ }^{1}$; Couture, Frédéric'; Levesque, Christine ${ }^{1}$ Kwaitkowska, Anna ${ }^{1}$; Routhier, Sophie'; Desjardins, Roxane ${ }^{1}$; Guérin, Brigitte $^{2}$; Doueik, Alexandre ${ }^{3}$; Carmel, Michel ${ }^{3}$; Sabbagh, Robert ${ }^{3}$

'Institut de pharmacologie de Sherbrooke et Université de Sherbrooke, Sherbrooke, QC, Canada; ${ }^{2}$ Centre d'imagerie moléculaire de Sherbrooke et Université de Sherbrooke, Sherbrooke, QC, Canada; ${ }^{3}$ Centre hospitalier universitaire de Sherbrooke et Université de Sherbrooke, Sherbrooke, QC, Canada
Introduction and Objectives: Our studies provide increasing evidence for the role of the proprotein convertases (PCs) in prostate cancer. The PCs are proteolytic enzymes involved in precursor activation of many cancer-associated proteins. Our focus has been on one PC in prostate cancer, namely PACE4. Our data validates PACE4 as a potential therapeutic target and aims to understand its mechanisms in prostate cancer progression, while testing its molecular and pharmacologic silencing in cellular and animal models.

Methods: We examined PACE4 expression in prostate cancer tissues obtained from radical prostatectomies. We also studied three classic prostate cancer model cell lines, namely DU145, LNCaP and PC3 cells for PC expression and effects of downregulation via molecular silencing using lentivirus delivered shRNAs. We developed and tested PACE4 inhibitors (and analogs) as potential therapeutic leads. In vivo biodistribution kinetics were measured in tumor-bearing Balb/c nude mice by $\mu$ PET imaging. Results: Our results demonstrate that PACE4 is over-expressed in $100 \%$ of the prostate cancers tissues tested to date, while levels correlate with Gleason scores. Molecular PACE4 silencing in cancer cells lines, results in highly reduced proliferation in vitro, but also when these modified cell lines are implanted in immuno-deficient mice. Pharmacologic PACE4 inhibitors reduced growth rates of prostate cancer cell lines when tested in xenograft mouse models. A PACE4 imaging probe detects PACE4 cancer cells lines implanted in immuno-deficient mice.

Conclusions: Our data shows that PACE4 is a key enzyme in prostate cancer. PACE4 is most likely activating key growth factors, and the lack of active growth factors can severely limit further proliferation. Further studies will focus on the feasibility of this approach as well as the limits in regards to potential cell resistance. The identification of these PC-related growth factors is currently being examined.

\section{UP-048}

Histopathological Features Following Prostate High Intensity Focus Ultrasound Salvage Therapy for Radiation-failure

Autran Gomez, Ana Maria; Chang, Susanne; Gomez-Lemus, Jose; Lee, Linda; Izawa, Jonathan; Chin, Joseph

London Health Sciences Centre, University of Western Ontario, London, ON, Canada

Introduction and Objectives: The interpretation of needle biopsy specimens from prostates following radiotherapy is often challenging. In locally radio-recurrent prostate cancer, high intensity focused ultrasound (HIFU) is a promising salvage treatment option. Biopsy specimens post-HIFU are even more challenging to interpret. Herein, we document the histologic features following whole-gland salvage HIFU.

Methods: From 2006 to 2010, 55pts, with biopsy-proven localized radiorecurrent prostate cancer $(\mathrm{PCa})$ were subjected to whole- gland salvage HIFU.TRUS-guided prostatic biopsies were routinely performed at 6 mos.

\begin{tabular}{|c|c|c|c|c|c|}
\hline & & $\begin{array}{c}\text { Baseline PSA }>10 \\
(n=44)\end{array}$ & $\begin{array}{c}\text { PSA rise }>10 \\
(n=80)\end{array}$ & PSA $\leq 10(n=216)$ & $p$-value \\
\hline Median follow-up months (IQR) & & $33.9(18.3-63.4)$ & $54.7(36.3-73.7)$ & $43.5(22.8-62.9)$ & 0.03 \\
\hline PP at 1 st repeated biopsy(\%) & & 14 (31.8) & $28(35.0)$ & $60(27.8)$ & 0.44 \\
\hline \multirow[t]{2}{*}{ Reason for PP at 1st repeat biopsy } & $\begin{array}{c}\text { Number of cores involved } \\
(\%)\end{array}$ & $6(13.6)$ & $15(18.6)$ & $38(17.6)$ & 0.76 \\
\hline & $\%$ core involvement $(\%)$ & $3(6.8)$ & $7(8.8)$ & $15(6.9)$ & 0.86 \\
\hline Number who underwent treatment (\%) & & $20(45.5)$ & $30(37.5)$ & $74(34.3)$ & 0.36 \\
\hline PP at subsequent biopsy (\%) & & $1(2)$ & $4(5)$ & $9(4)$ & 0.19 \\
\hline $\begin{array}{l}\text { Number started on 5-alpha reductase } \\
\text { inhibitor }(\%)\end{array}$ & & $8(18.2)$ & 27 (33.8) & $48(22.2)$ & 0.007 \\
\hline
\end{tabular}




\begin{tabular}{lcc}
\hline Table 1. UP-048 & $\begin{array}{c}\text { Pre HIFU Biopsies } \\
\text { (N=55) }\end{array}$ & $\begin{array}{c}\text { Post HIFU Biopsies } \\
\text { (N=14) }\end{array}$ \\
\hline Parameters & $69(57-79)$ & $71(67-74)$ \\
\hline Age yr & $3.61(0.10-15.8)$ & $4.25(2.61-6.08)$ \\
PSA ng/ml & & \\
Gleason Score (n\%) & $16(29)$ & \\
$\leq 6$ & $28(51)$ & $8(57)$ \\
7 & $11(20)$ & $2(14)$ \\
$\geq 8$ & & $4(29)$ \\
N/D & & $3(21)$ \\
Localization n(\%) & $24(44)$ & \\
Base & $8(14)$ & $4(29)$ \\
Mid & $1(2)$ & $2(14)$ \\
Apex & $2(4)$ & \\
Mid+Apex & $12(22)$ & $5(36)$ \\
Mid+Apex+Base & $8(14)$ & \\
Mid+Base & & \\
\hline Note: Median (range). HIFU: high intensity focused ultrasound; PSA: prostate-specific \\
antigen.
\end{tabular}

post treatment. This study included the retrospective histopathological review, by one expert Uro-pathologist, of all prostatic biopsy performed. Immunohistochemical stains and high molecular weight cytokeratin were performed.

Results: Median follow-up was 25 months (5-56). Of 55 pts, 49(89\%) underwent a standardized follow-up biopsy. Median pre-salvage PSA was $3.61 \mathrm{ng} / \mathrm{ml}$ and post-salvage nadir was $0.19 \mathrm{ng} / \mathrm{ml}$. Biopsy was positive in 14 cases. Clinical characteristics listed in Table 1. In all cases, benign prostate ducts and acini showed variable degrees of atrophy. Treatment related changes included marked reactive atypia (93\%) edema, fibrosis and cystic changes $(71 \%)$ and coagulative necrosis $(86 \%)$. Concordance of Gleason grading was observed between the pre- and post-therapy specimens in $60 \%$ cases, upgraded (+1 Gleason score) in 30\% pts and downgraded (-3 Gleason score) in 10\%. Extent of disease in those with persistent cancer showed significantly lower cancer extent, with many having focal or limited uni-lobar involvement. HPIN was still seen in 8 (57\%) biopsies post-HIFU.

Conclusions: The histopathological analyses of prostate biopsies following whole-gland salvage HIFU require an expert Uro-pathologist for accurate interpretation and diagnosis, which is an integral part of the management of patient with radio-recurrent prostate cancer.

\section{UP-049}

Insight into Dietary Prevention of Prostate Cancer: Effects of Fish Oil and Krill Oil on Inflammation

Moreel, Xavier; Dong, Ruijing; Julien, Pierre; Fradet, Vincent

Laval University, Quebec, QC, Canada

Introduction and Objectives: Inflammation is a major risk factor for prostate cancer (PCa). Our previous data showed that, between patients, prostatic inflammation is highly variable and that a high level is associated with aggressive $\mathrm{PCa}$. Here, we aimed to compare incorporation of fish oil (FO) and krill oil (KO) into prostatic epithelial cells and their impact on inflammation level.

Methods: Primary prostatic epithelial cells were derived from a radical prostatectomy specimen for Gleason 7 organ-confined PCa. Biopsies were taken from the normal prostatic zones and were cultured in selective medium (KSFM) to isolate prostatic epithelial cells. At $90 \%$ confluence, $\mathrm{FO}$ or $\mathrm{KO}$ or control buffer was added to the medium for 24 hours. Prostatic inflammation level was characterized by measuring secreted IL-8 in the medium and was normalized to the number of cells based on total DNA. After trypsinization of prostatic cells, 4-cycle washing with HBSS and lipid extraction of cell membranes, fatty acid profiles were obtained by gas chromatography. All experiences were triplicated.
Results: FO and KO increased the levels of omega-3 (13-fold, $p<0.0001$; 12 -fold, $p=0.003)$ but only slightly of omega- 6 (1,5- and 1,2-fold; both $p>0.08$ ) fatty acids in cultured prostatic cells. Fatty acids from KO (phospholipids) were incorporated faster into cell membrane than fatty acids from FO (triglycerides). However, the addition of FO or KO increased the levels of inflammation in cells from this patient with a low prostatic inflammation level.

Conclusions: Integration into prostate cell membrane of fatty acids from $\mathrm{KO}$ is faster than from $\mathrm{FO}$, because of their molecular type. In this patient with low inflammation level, both oils increased the inflammation level, likely because of the omega- 6 content of both oils. Further work to decipher how omega- 3 and -6 fatty acids affect prostate inflammation is needed. Prevention of PCa by dietary supplements of FO or KO may not be adapted to every patient.

\section{UP-050}

Inconsistent Testosterone Monitoring and Antiandrogen Use in Prostate Cancer Patients Receiving Androgen Deprivation Therapy

Dalla Nora, Sergio'; Shayegan, Bobby ${ }^{2}$

${ }^{1}$ Ferring Inc., North York, ON, Canada; ${ }^{2}$ Juravinski Cancer Centre, McMaster University, Hamilton, ON, Canada

Introduction and Objectives: The goal of androgen deprivation therapy is the suppression of testosterone $(\mathrm{T})$ to castrate levels. Despite this goal, routine monitoring of $\mathrm{T}$ and antiandrogen (AA) use to prevent $\mathrm{T}$ surge and clinical flare in patients receiving gonadrotropin-releasing hormones $(\mathrm{GnRH})$ agonists appears to be inconsistent. A series of discussions involving surveys using an audience response system that ensures anonymity was held in order to identify and quantify gaps in clinical practice.

Methods: From May 3 to November 24, 2011, 15 discussion groups sponsored by Ferring Inc. were held across Canada. Mostly urologists and some radiation oncologists ( $n=133$, range 6-11 per discussion group) were surveyed pre- and post-discussion on the role of T monitoring and the impact of inadequate T suppression on disease progression and survival as reported in the literature. Questions included definition of castrate levels, frequency of T monitoring and AA use for flare protection.

Results: Ranges are between discussion groups. Pre-discussion, 53\% (range $0-91 \%)$ indicated $0.5 \mathrm{ng} / \mathrm{mL}(1.7 \mathrm{nmol} / \mathrm{L})$ was an adequate castrate level; post-discussion, $91 \%(75-100 \%)$ chose either 0.32 or $0.2 \mathrm{ng} /$ $\mathrm{mL}$. Pre-discussion, $24 \%(0-83 \%)$ indicated they measured T at baseline, periodically and with rising PSA; post-discussion, 60\% (12-100\%) would do so. Pre-discussion, 51\% (10-100\%) indicated they always use AA for flare protection.

Conclusions: Frequency of testosterone monitoring, interpretation of castrate levels and antiandrogen use in prostate cancer patients receiving androgen deprivation therapy varies widely between physicians and across Canada. These data indicate a need to provide better education and guidance on timing, interpretation and management of testosterone measurements.

\section{UP-051}

Single Nucleotide Polymorphisms (SNPs) Associated with Late Radiation Toxicity after Prostate Brachytherapy

Leong, Nelson ${ }^{1}$; Parliament, Matthew ${ }^{1}$; Martell, Kevin ${ }^{2}$; Ghosh, Sunita ${ }^{2}$; Pervez, Nadeem ${ }^{1}$; Pedersen, John'; Yee, Don'; Murtha, Albert'; Amanie, John'; Usmani, Nawaid ${ }^{1}$

${ }^{1}$ Cross Cancer Institute, Edmonton, AB, Canada; ${ }^{2}$ University of Alberta, Edmonton, $\mathrm{AB}$, Canada

Introduction and Objectives: Excessive toxicity from prostate brachytherapy treatment may be related to increased radiosensitivity from genetic polymorphisms. We aimed to identify single nucleotide polymorphisms (SNPs) that were associated with high toxicity after therapy to determine possible markers for increased radiation sensitivity.

Methods: 349 patients treated with prostate brachytherapy at the Cross Cancer Institute between 1998 - 2010 provided saliva samples from which DNA was extracted for this research ethics board approved study. In the cohort of patients with at least 2 years of follow-up, 41 patients were identified as having high late toxicity ( $\geq$ RTOG grade 2 Gl or GU toxicity). 
71 patients were identified as controls with minimal toxicity (persistent IPSS increase $<6$, EPIC quality of life score $>79$ ). We analyzed 15 potential SNPs from 13 genes (MSH6, GSTA1, SOD2, NOS3, GSTP1, ATM, LIG4, XRCC1, XRCC3, RAD51, TP53, TGFB1, ERCC2) for correlation with increased late toxicity. Patient factors and dosimetric parameters were also collected and included in the analysis.

Results: All 15 proposed SNPs demonstrated polymorphism. We implemented a univariate analysis to examine the correlation between variant allele SNPs and increased toxicity. This revealed a statistically significant relationship in 5 of the SNPs. Variants in SNPs rs1695 (GSTP1), rs1800470 (TGFB1), rs1801320 (RAD51), rs1805386 (LIG4), and rs4880 (SOD2) were correlated with increased late toxicity (all $p<0.1$ ). Diabetes, coronary disease, hormone therapy, age at implantation and parameters such as the prostate $\mathrm{V} 150, \mathrm{~V} 100$, and D90 were also all significant for an association with late toxicity $(p<0.1)$.

Conclusions: Our study demonstrates 5 SNPs in GSTP1, TGFB1, RAD51, LIG4 and SOD2 which are statistically significant for association with late toxicity, which may be utilized in future studies to identify patients at risk for complications after brachytherapy.

\section{UP-052}

Prostate Cancer Antigen 3 Density Shows Similar Predictive Value of Positive Prostate Biopsy as Prostate Cancer Antigen 3 Alone

Dovirak, Ostap; Goode, Roland; Marshall, Susan; Consiglio, Joseph; Duff, Michael; Chevli, K. Kent

State University of New York at Buffalo Department of Urology, Buffalo, NY, United States

Introduction and Objectives: Prostate Cancer Antigen (PCA3) is a novel biomarker currently used for improved prediction of prostate cancer on prostate biopsy. Multiple studies have indicated superior specificity of PCA3 when compared to PSA levels alone. Also, PCA3 has been shown to be directly related to tumor burden in prostate cancer, suggesting that higher PCA3 values relative to the volume of the prostate indicate greater tumor burden. This represents the concept of PCA3 denisty. Studies have demonstrated improved predictive ability of PCA3 density for prostate cancer on prostate biopsy. We evaluated this hypothesis in our patient population.

Methods: PSA levels, PCA3 levels and prostate volumes were collected from 1152 men who had prostate biopsy in a retrospectively organized database from a single organization. All men had PCA3 urine assay performed after a deliberate prostate exam immediately before undergoing trans-rectal ultrasound and prostate biopsy. Prostate volume was calculated using the length $\mathrm{x}$ width $\mathrm{x}$ height method during trans-rectal ultrasound. Receiver operating characteristic (ROC) curves were plotted and the area under the curve (AUC) was determined.

Results: 798 out of 1152 patients included in the study had a positive prostate biopsy. Logistic regression was used to generate area under the ROC curve for PCA3, PSA and PCA3 density. Our analysis showed the AUC for PSA, 0.5400, AUC for PCA 3, 0.6816 and AUC for PCA3 density, 0.6803 . The difference in the AUC for PCA3 and PCA3 density was not statistically significant.

Conclusions: In this study, there was no statistical difference in prediction of positive prostate biopsy between PCA3 and PCA3 density.

\section{UP-053}

Use of Patient Educational Technologies (PET) by Patients Diagnosed with Prostate Cancer

Carlson, Kevin ${ }^{1}$; Crump, R. Trafford ${ }^{2}$; Baverstock, Richard

${ }^{1}$ University of Calgary, Calgary, $\mathrm{AB}$, Canada; ${ }^{2}$ University of British Columbia, Centre for Health Services and Policy Research, Vancouver, BC, Canada

Introduction and Objectives: Patient education is a vital part of successful shared decision making. Conventional educational materials provide limited feedback as to their use. Information technology, however, offers opportunities for more interactive platforms and a richer understanding of their effectiveness. We set out to study how patients interact with Patient
Educational Technology (PET) aimed at informing them about their treatment options for prostate cancer.

Methods: A PET library was developed for prostate cancer, with 15 modules falling into 3 categories: those related to diagnosis (4), treatment options (8), and treatment side effects (3). Data generated from patients' use of PET is tracked in detail and recorded in real time. The PET library was made available to the patient population of two urologists between 2008 and 2011.

Results: 394 patients newly diagnosed with prostate cancer were given access to the PET library. 123 (31\%) with a mean age of 56.4 logged in and viewed at least one module (mean 3, range 1-12). On first visit patients most commonly viewed material on diagnosis, while subsequent visits focused on treatment. Specific information sought shifted from clinical and procedural to that of risk, side effects and decision making with later visits. The average time viewing material on first visit was 10 minutes versus 14 minutes for return visits $(p<0.025)$. The average time over which patients viewed modules was 43 days.

Conclusions: When referred, a number of patients diagnosed with prostate cancer engage with PETs on an ongoing basis between the time of diagnosis and treatment. The information sought evolves in a logical manner. Understanding which patients engage with these types of technologies, and how, is an important step in developing more useful PETs; tools that hold the potential to provide an effective and inexpensive means to improve shared decision making on a broad scale.

\section{UP-054}

Comparison of Pathological Outcomes of Radical Prostatectomy in African Americans and Caucasians Who Are Potential Candidates for Active Surveillance

Popovic, Mihailo; Lucas, Steven

Wayne State University, School of Medicine, Urology, Detroit, MI, United States

Introduction and Objectives: While active surveillance is safe in appropriately selected patients, there is little data to show whether African Americans (AA), who may represent a higher risk population, have equivalent outcomes as other racial groups. We compared pathological outcomes of AA and Caucasians who underwent radical prostatectomy (RP) but who were candidates for active surveillance (AS).

Methods: A retrospective database review was conducted on patients that underwent RP between June, 1990 and March, 2011. Patients were considered potential candidates for AS if their biopsy Gleason scores were $6<=$ and clinical stage T2a or less. Intermediate risk patients were considered to have either stage $\mathrm{T} 2 \mathrm{~b}$ or Gleason score $3+4$.

Results: A total of 1,444 (47.5\%) were Caucasian, 1,416 (46.6\%) AA and $177(5.8 \%)$ other. A total of $1,139(37.5 \%)$ were potential candidates for AS following biopsy, and $761(25.1 \%)$ were intermediate risk. Caucasians more frequently met the criteria than $\mathrm{AA}(40.0 \%$ vs. $35.5 \%, p=0.03)$. At $\mathrm{RP}$, Caucasians and $\mathrm{AA}$ demonstrated a similar rate of Gleason upgrading: $334 / 570(58.6 \%)$ vs. $291 / 496(58.7 \%), p=0.981$. A primary Gleason grade 4 was observed in $20.3 \%$ and $28.9 \%$ of Caucasians and AA respectively, $p=0.890$. Positive margins were noted in $162(29.6 \%)$ Caucasians and $173(36.7 \%)$ AA, $p=0.016$. Seminal vesicle (SV) invasion was noted in $46(8.5 \%)$ Caucasians and $46(10.0 \%) \mathrm{AA}, p=0.420$. Among intermediate risk patients, $92 / 378(24.3 \%)$ of Caucasians were upgraded to primary Gleason 4 vs. $79 / 329(24.0 \%)$ of $A A, p=0.919$. Positive margins were observed in $36.2 \%$ of Caucasians and $40.4 \%$ of $A A, p=0.527$. SV invasion was observed in $12.0 \%$ Caucasians and $14.7 \% \mathrm{AA}, p=0.290$.

Conclusions: Using loose criteria for AS, both Caucasians and AA had a similar rate of eligibility. We found that AA race, with exception of margin status in low risk patients, was not a risk factor for adverse pathological outcome at RP in low and intermediate risk AS patients. 


\section{UP-055}

Ontario's First 18f Choline Prostate PET Images: Histology and Multi-parametric MRI Correlation

Gibson, Eli'; Rachinsky, Irina ${ }^{2}$; Gaed, Mena ${ }^{3}$; Romagnoli, Cesare ${ }^{2}$; Lee, Ting-Yim¹; Gómez, José A. ${ }^{4}$; Moussa, Madeleine ${ }^{4}$; Crukley, Cathie" ${ }^{3}$ [ Chin, loseph $^{5}$; Bauman, Glenn S. ${ }^{6}$; Ward, Aaron D. ${ }^{7}$

${ }^{1}$ Robarts Research Institute, The University of Western Ontario, London, ON, Canada; ${ }^{2}$ Department of Medical Imaging, The University of Western Ontario, London, ON, Canada; ${ }^{3}$ Lawson Health Research Institute, London, ON, Canada; ${ }^{4}$ Department of Pathology, The University of Western Ontario, London, ON, Canada; ${ }^{5}$ Department of Urology, The University of Western Ontario, London, ON, Canada; ${ }^{6}$ Department of Oncology, The University of Western Ontario, London, ON, Canada; ${ }^{7}$ Department of Medical Biophysics, The University of Western Ontario, London, ON, Canada

Introduction and Objectives: Localising and grading prostate cancer (PCa) on in vivo imaging could support diagnosis, therapy selection, and possibly focal therapy guidance. Multi-parametric MRI (mpMRI) is being evaluated for these uses, but identifying PCa foci can still be challenging even with mpMRI. ${ }^{18} \mathrm{~F}$-fluorocholine $(18 \mathrm{FCH})$ PET has also shown promise for locating PCa. We have established a robust process for registering in vivo prostate images to post-prostatectomy digital histology images. We have recently introduced in vivo $18 \mathrm{FCH}$ PET imaging in an REB-approved prospective study of pathologic validation of multi-modality PCa imaging. We present preliminary progress using what we believe are Ontario's first $18 \mathrm{FCH}$ prostate $\mathrm{PET}$ images.

Methods: We acquired 3T mpMRI (DW, T2W, and dynamic contrast T1W) and $18 \mathrm{FCH}$ PET/CT images of a subject as part of our prospective study. Visible lesions on post-surgery hematoxylin \& eosin-stained histology images were contoured and graded. Our software performed 2D-3D affine registrations of histology to ex vivo MRI with $0.7 \mathrm{~mm}$ error. We registered the reconstructed $3 \mathrm{D}$ histology image to in vivo PET and mpMRI images, via rigid and thin-plate spline transformations, respectively.

Results: We observed Gleason 3 and 4 PCa foci that were challenging to identify on mpMRI and corresponded to higher $18 \mathrm{FCH}$ uptake (SUVmax: foci 3.5-5.4 cf bkg. 2.5) on PET.

Conclusions: $3 \mathrm{D}$ registration of mpMRI, $18 \mathrm{FCH}$ PET and histology suggests an incremental PCa detection and localisation benefit of $18 \mathrm{FCH}$ PET compared to mpMRI alone for our first subject imaged with both modalities. For such patients with $18 \mathrm{FCH}$ PET-avid and mpMRI-isointense lesions, $18 \mathrm{FCH}$ PET could be promising with great potential for diagnosis and focal therapy guidance.

\section{UP-056}

Laparoscopic Robot-assisted Extended Pelvic Lymph Node Dissection for High Risk Prostate Cancer

Bladou, Franck'; Luz, Murillo²; Anidjar, Maurice ${ }^{2}$

${ }^{1}$ McGill University, Jewish General Hospital, Montreal, QC, Canada; ${ }^{2}$ McGill University, Montreal, QC, Canada

Introduction and Objectives: Radical prostatectomy is indicated for selected patients with high-risk prostate cancer. During the procedure, an extended lymph node dissection should be performed for staging purpose. A laparoscopic approach has been proposed for this procedure but has been limited to experienced centres, due to the technical difficulty and the vascular risk of an extended lymph node dissection.

Methods: Robotic-assistance is an alternative of pure laparoscopic procedure and can be proposed to enhance the surgeon gesture in a limited anatomic area while keeping oncological and surgical safety.

Results: The video shows a step-by-step laparoscopic robot-assisted extended lymp node dissection. The robotic assistance offer a 3-D surgical vision and a precise gesture thanks to the endowrist technology of the DaVinci instruments. A complete dissection of the common iliac, external iliac and obturator fossa and internal iliac lymph node dissection is shown, with anatomical landmarks for an ideal template.

Conclusions: Robot-assisted extended pelvic lymph node dissection can be performed as well as the standart open procedure with the same oncological safety.

\section{UP-057}

Comparative Morbidity between Salvage High Intensity Focused Ultrasound and Cryotherapy for Radiorecurrent Prostate Cancer Al-Zahrani, Ali; Yutkin, Vladimir; Autran-Gomez; Ana Maria; Chin, Joseph Division of Urology, Department of Surgery, London Health Sciences Centre, University of Western Ontario, London, ON, Canada

Introduction and Objectives: High intensity focused ultrasound (HIFU) utilizes focused ultrasound waves to destroy tissues. Wither as primary or salvage modality, HIFU is increasingly being promoted in the management of prostate cancer ( $\mathrm{PCa}$ ). Our primary objective is to assess the adverse events rate after salvage HIFU in patients with radiorecurrent PCa. We compared the results with salvage Cryotherapy (CRYO) adverse events which is another minimal invasive modality.

Methods: We retrospectively reviewed the adverse events of all patients who underwent salvage HIFU for recurrent PCa after Radiotherapy (20062010). The first equal cohort of patients who underwent salvage CRYO was selected for comparison (1995-1998).

Results: Salvage HIFU had lower incontinence $(4.6 \%$ vs. $53 \%)$ and urinary retention rate $(6.2 \%$ vs. $28 \%)$. Perineal pain rate was lower in the HIFU group ( $5 \%$ vs. $24 \%$ ). There was no bladder contracture in the HIFU group. The rate of postoperative hematuria was similar between both

\begin{tabular}{|c|c|c|c|}
\hline Variables & Salvage HIFU 2006-2010 (n=64) & Salvage Cryotherapy 1995-1998 $(n=64)$ & $p$ Value \\
\hline Age & 67 & 66 & NS \\
\hline Pre Salvage PSA & 3.1 & 9.2 & $<0.05^{*}$ \\
\hline Incontinence (mild/moderate) & $3(4.6 \%)$ & $34(53 \%)$ & $<0.05^{* *}$ \\
\hline Incontinence requiring surgery & $1(1.5 \%)$ & $2(3.1 \%)$ & NS \\
\hline Perineal pain & $3(5 \%)$ & $14(24 \%)$ & NS \\
\hline Recto-urethral Fistula & $2(3.1 \%)$ & $1(1.5 \%)$ & NS \\
\hline Urinary Retention & $4(6.2 \%)$ & $18(28 \%)$ & $<0.05^{* *}$ \\
\hline Gross Hematuria & $7(11 \%)$ & $7(11 \%)$ & NS \\
\hline Urethral Sloughing & $1(1.5 \%)$ & $2(3.1 \%)$ & NS \\
\hline Bladder Neck Contracture & 0 & $6(9.3 \%)$ & $<0.05^{* *}$ \\
\hline Urinary Tract Infection & $6(9.3 \%)$ & $8(12.5 \%)$ & NS \\
\hline
\end{tabular}

* Mann Whitney test; ** Fisher Exact test; HIFU: high intensity focused ultrasound; PSA: prostate-specific antigen. 
modalities. Recto-Urethral fistula rate and urethral sloughing were low in both modalities (3.1\% and $1.5 \%, 1.5 \%$ and $3.1 \%$, respectively) (Table 1 ). Conclusions: HIFU is a feasible salvage procedure in patients with radiorecurrent PCa. Salvage HIFU has lower adverse events in comparison to salvage CRYO in this group of patients.

\section{UP-058}

A Nomogram Containing the NF-kappa B p65 Biomarker Predicts Biochemical Recurrence of Prostate Cancer Patients Following Radical Prostatectomy

Gannon, Philippe O. ${ }^{1}$; Lessard, Laurent ${ }^{1}$; Stevens, Louis-Mathieu ${ }^{2}$; $\underline{L a b o u b a, ~ I n g r i d ~}^{1}$; Forest, Valérie ${ }^{1}$; Bégin, Louis R. ${ }^{3}$; Minner, Sarah ${ }^{4}$; Tennstedt, Pierre ${ }^{5}$; Schlomm, Thorsten ${ }^{5}$; Mes-Masson, Anne-Marie ${ }^{6}$; Saad, Fred $^{7}$

${ }^{1}$ Centre de recherche du Centre hospitalier de l'université de Montréal (CRCHUM), Montreal, QC, Canada; ${ }^{2}$ Division of Cardiac Surgery, CHUM, CRCHUM, Montreal, QC, Canada; ${ }^{3}$ Division of Anatomic Pathology, Hopital Sacré-Coeur de Montréal, Montreal, QC, Canada; ${ }^{4}$ Institute of Pathology, University Medical Center Hamburg-Eppendorff, Hamburg, Germany; ${ }^{5}$ Martini-Clinic, Prostate Cancer Center, University Medical Center Hamburg-Eppendorff, Hamburg, Germany; ${ }^{6} \mathrm{CRCHUM}$, Institut du cancer de Montréal, Department of Medicine, Université de Montréal, Montreal, QC, Canada; ${ }^{7}$ Division of Urology, CHUM, Université de Montréal, CRCHUM, Montreal, QC, Canada

Introduction and Objectives: The robust association between the nuclear localization of NF-KB p65 and poor prognosis is well documented in prostate cancer $(\mathrm{PCa})$. However, the potential application of NF-кB p65 as PCa prognostic marker in clinical settings is not yet addressed. Here, we tested NF-кB p65 as variable in clinical nomogram predicting the biochemical recurrence (BCR) to improve the predictive accuracy.

Methods: Primary tumors from radical prostatectomy from a large European PCa cohort served for building tissue microarrays. NF- $\mathrm{kB}$ p65 expression was detected by immunohistochemistry on 2,084 cores containing suitable malignant tissue. NF-кB p65 nuclear frequency and its cytoplasmic intensity were quantified by an uro-pathologist. An epidemiologist revisited statistical analyses.

Results: Overall, our analyses confirmed the association between NF- $\mathrm{KB}$ p65 and PCa severity. Patients with nuclear NF-кB p65 expression had an increased frequency of BCR $(p<0.001)$, metastasis $(p=0.002)$, and mortality $(p=0.005)$. A novel variable considering the inactive cytoplasmic and transcriptionally active nuclear NF- $\mathrm{kB}$ p65 fraction was a strong predictor of $\mathrm{BCR}$ in a Cox regression multivariate model. Then we developed a nomogram for the prediction of $\mathrm{BCR}$ at 12,60 and 120 months after radical prostatectomy. The inclusion of NF-кB p65 variable to the base clinical multivariable model improved significantly the predictive accuracy of the nomogram that was increased from 80.51 to $80.74 \%(p=0.004)$.

Conclusions: We confirmed that NF- $\mathrm{KB}$ p65 predicts the BCR in univariate and multivariate models. The inclusion of NF-KB p65 in nomogram presents a clinical benefit for the prediction of BCR and may improve the decision-making process for more adapted therapies of PCa patients.

\section{UP-059}

Management of Post Radiation Therapy Complications among Prostate Cancer Patients: A Single Surgeon Experience Flannigan, Ryan'; Baverstock, Richard ${ }^{2}$

${ }^{1}$ University of British Columbia, Vancouver, BC, Canada; ${ }^{2}$ vesia [Alberta Bladder Centre], Division of Urology, University of Calgary, Calgary, $A B$, Canada

Introduction and Objectives: While treating prostate cancer with radiation therapy (RT) has proven to be a viable option, some of the resulting complications serve to be challenging to manage and are referred for urologic opinion. We describe a single surgeon experience in the management of post prostate cancer radiotherapy complications.

Methods: Patients previously receiving External Beam Radiation (XRT) or Brachytherapy (BT) presenting back with Urology related difficulties between 2005 and 2010 were retrospectively analyzed. Full hospital and clinic EMR chart reviews were performed.
Results: 15 patients were identified, mean age of 68.9 years, presenting with a total of 42 complications. 10 (67\%) patients (27 complications; $64 \%$ ) had previously received XRT, and 5 (33\%) (15 complications; $36 \%)$ patients had previously received BT. Of these complications, $24(57 \%)$ were obstructive, $11(26 \%)$ were hematuria, $5(12 \%)$ were incontinence, and $2(5 \%)$ were infections. Upon investigating these patients, 34 (81\%) cystoscopies were performed, $6(14 \%)$ urodynamic tests, $1(2 \%)$ prostatic biopsy, and $1(2 \%)$ computed tomography. Managing these patients often required multiple modalities of therapy. Surgical management was used $27(41 \%)$ times, local therapy $18(28 \%)$ times, and medical therapy $20(31 \%)$. The resulting condition of these patients includes $3(20 \%)$ patients that have incontinence, 3 $(20 \%)$ that self catheterize, $2(13 \%)$ report voiding well, $2(13 \%)$ who underwent cystectomy with ileo-conduits, $1(7 \%)$ with an indwelling catheter, 1 $(7 \%)$ with continued obstructive voiding, and $3(20 \%)$ awaiting follow-up. Conclusions: Among RT complicated patients referred for urologic management, the majority suffer from obstructive voiding difficulties, and hematuria. These patients are heavily investigated and require significant resources in their care to optimize their condition. Several treatment modalities are being utilized to treat these patients, and improve their quality of life.

\section{UP-060}

Salvage High Intensity Focused Ultrasound for Local Prostate Cancer Recurrence after Brachytherapy

Al-Zaharni, Alii ${ }^{1}$; Yutkin, Vladimir ${ }^{2}$; Autran-Gomez, Ana Maria ${ }^{2}$; Chin, Joseph $^{2}$

${ }^{1}$ London Health Sciences Centre, London, ON, Canada; ${ }^{2}$ Division of Urology, Department of Surgery, London Health Sciences Centre, University of Western Ontario, London, ON, Canada

Introduction and Objectives: Management of patients with recurrent Prostate Cancer ( $\mathrm{PCa}$ ) after Brachytherapy is challenging. These patients might benefit from definitive local therapy. The objective of this study is to assess the safety and feasibility of high intensity focused ultrasound (HIFU) in this group of patients.

Methods: Patients with salvage HIFU for local PCa recurrence after brachytherapy were retrospectively reviewed. All participants had their treatment with (Sonablate ${ }^{\circledR}$ 500) between 2006 and 2011. Patients were followed with prostate-specific antigen (PSA) and transrectal ultrasound guided biopsy. Progression was defined as positive biopsy and/or PSA $>$ nadir $+2 \mathrm{ng} / \mathrm{ml}$.

Results: Thirteen patients underwent salvage HIFU for PCa recurrence after brachytherapy. The mean age was 66.5 year (58.8-72.5) and the

\section{Table 1. UP-060. Clinical and pathological characteristics} before both brachytherapy and salvage HIFU

\begin{tabular}{lcccc}
\hline \multirow{2}{*}{ Variables } & \multicolumn{2}{c}{ Brachytherapy } & \multicolumn{2}{c}{ Salvage HIFU } \\
& Mean & Range & Mean & Range \\
\hline Base line PSA (ng/dl) & 7.1 & $4.4-11.7$ & 4.4 & $1.1-9$ \\
Prostate Volume (cc) & 32 & $25-39$ & 22.6 & $13-40$ \\
Gleason Score & 6 & 6 & 7 & $6-8$ \\
Nadir PSA (ng/dl) & 0.62 & $0.18-1.24$ & 0.97 & $0-4$ \\
Time to Nadir (months) & 41.8 & $7.1-75.1$ & 3.17 & $1.5-6$ \\
\hline HIFU: high intensity focused ultrasound; PSA: prostate-specific antigen.
\end{tabular}

Table 2. UP-060. Adverse events rate after salvage HIFU

\begin{tabular}{lc}
\hline Adverse events & Rate (\%) \\
\hline Temporarily worsening lower urinary tract symptoms & $9(75 \%)$ \\
Gross hematuria & $3(25 \%)$ \\
Recto-urethral fistula & $2(16.6 \%)$ \\
Urinary retention & $2(16.6 \%)$ \\
Perineal pain & $1(8.3 \%)$ \\
\hline HIFU: high intensity focused ultrasound.
\end{tabular}


mean follow-up was 33.7 months (3-68). The clinical and pathological characteristics before brachytherapy and salvage HIFU are summarized in Table 1. All transrectal ultrasound guided biopsy at 6 months post salvage HIFU were negative. The biochemical failure rate was $50 \%$. Additional therapy was required in $50 \%$ of patients. The comments adverse event was temporarily worsening lower urinary tract symptoms $(75 \%)$ (Table 2 ). Rectourethral fistula rate was $16.6 \%$.
Conclusions: Salvage HIFU is a feasible treatment option for PCa recurrence post brachytherapy with acceptable morbidity rate. Larger cohort of patient and longer follow-up should be carried out to confirm the findings.

\section{Table 1. UP-061}

\begin{tabular}{|c|c|c|c|}
\hline & Group 1 & Group 2 & $p$ value \\
\hline No. & 53 & 27 & \\
\hline \multicolumn{4}{|l|}{ PREOPERATIVE DATA } \\
\hline Indications: & 0.11 & & \\
\hline - Retention & 5 & 7 & \\
\hline • LUTS & 47 & 20 & \\
\hline ASA score: & & & 0.49 \\
\hline$\cdot 1$ & 36 & 19 & \\
\hline$\bullet \|$ & 11 & 7 & \\
\hline$\cdot$ •III & 6 & 1 & \\
\hline DM & $6(11.3 \%)$ & $6(22.2 \%)$ & 0.2 \\
\hline Preoperative anticoagulants & 14 & 5 & 0.6 \\
\hline Preoperative Q-max & 8.3 & 7.9 & 0.91 \\
\hline Preoperative PVR & 113.5 & 100.5 & 0.75 \\
\hline Preoperative IPSS & 16.2 & 16.2 & 0.34 \\
\hline Preoperative QOL & 3.4 & 3.3 & 0.33 \\
\hline Mean PSA $(\mathrm{ng} / \mathrm{mL} \pm \mathrm{SD})$ & 3.3 & 2.6 & 0.38 \\
\hline Mean TRUS volume of the gland $(\mathrm{mL} \pm \mathrm{SD})$ & 21.4 & 36.8 & 0.005 \\
\hline \multicolumn{4}{|l|}{ OPERATIVE DATA } \\
\hline Mean energy utilized $(\mathrm{KJ} \pm \mathrm{SD})$ & 54.3 & 53.1 & 0.923 \\
\hline $\mathrm{E} / \mathrm{P}$ ratio $=$ Energy $/$ preoperative $\mathrm{TRUS}(\mathrm{KJ} / \mathrm{mL} \pm \mathrm{SD})$ & 2.8 & 1.4 & 0.01 \\
\hline \multicolumn{4}{|l|}{ - Low grade (Clavien 1-2) } \\
\hline$\checkmark$ Retention/catheterization & 1 & - & \\
\hline$\checkmark$ Recurrent UTI & 2 & 1 & \\
\hline \multicolumn{4}{|l|}{ - High grade (Clavien 3-5) } \\
\hline$\checkmark$ BNC/Re-incision & - & 2 & \\
\hline$\checkmark$ Uretheral stricture/meatotomy & 2 & - & \\
\hline$\checkmark$ Uretheral stricture/urethrotomy & 1 & - & \\
\hline Late developed prostate cancer & - & 1 & 0.337 \\
\hline
\end{tabular}


UP-061

Holmium Laser Transurethral Incision of the Prostate (Hol-TUIP): Predictors of Long Term Outcome

Elshal, Ahmed; Elmansy, Hazem; Elhilali, Mostafa

McGill University, Urology Department, Montreal, QC, Canada

Introduction and Objectives: TUIP is a well-established treatment for small sized BPH (benign prostate hyperplasia). The size limitations and the long-term durability of success are ususally the main concern.

Methods: A retrospective review was done for 80 patients who underwent Hol-TUIP for symptomatic BPH. Patients were stratified into 2 groups, group-I with prostate size $<30$ c.c and group-II with prostate size $\geq 30$ c.c. All patients' variables as well as follow-up data were recorded and analyzed. Results: All preoperative parameters were comparable between both groups except for prostate size (Table 1). All patients had Hol-TUIP with deep incision of the bladder neck using laser energy to create a trough down to the capsule. No intraoperative complications or blood transfusion was recorded in both groups. Early and late adverse events were comparable in both groups $(p>0.05)$. After a median of 5.3 years follow-up, significant improvement of IPSS and Q-max was noted between preoperative values and at different follow-up points $(p<0.05)$. The percent change in Q-max and IPSS scores was comparable in both groups at different points however; the percent reduction in IPSS score was significantly less at five years in Group-II $(p<0.05)$. Overall reoperation rate was $6.2 \%$ (5 patients). Re-incision of the bladder neck was indicated in two patients in Group-II. Urethral stricture occurred in three cases in Group-I, where statistically significant more E/P ratio was utilized $(p<0.05)$. Retrograde ejaculation was reported in $31 \%$ of sexually active men with no significance difference between the two groups.

Conclusions: Hol-TUIP for treatment of $\mathrm{BOO}$ due to small sized prostate is a durable and efficient technique. The need for re-incision can be reduced by limiting the procedure to prostate size less than 30c.c. The relation between amount of energy used and its relevance to late sequelae is to be further studied.

\section{UP-062}

Evaluation of Autonomic Nervous System Activity by 24-Hour Holter Monitorization in Men with Benign Prostate Hyperplasia Zorba, Orhan Ünal; Uzun, Hakki; Cicek, Yuksel; Kirbas, Serkan; Erdogan, Turan; Yazar, Selim

Rize University, Rize Medical Faculty, Rize, Turkey

Introduction and Objectives: To analyze autonomic nervous system activity in patients with lower urinary tract symptoms due to benign prostate hyperplasia; we compared the results of 24-hour-heart rate variability between patients with benign prostate hyperplasia and controls.

Methods: A total of 67 men with lower urinary tract symptoms; storage (58.82 \pm 6.55 years) and voiding symptoms $(57.67 \pm 5.39$ years) were predominating in 18 and 49 patients respectively. 22 healthy men $(59.05 \pm 5.38$ years) were enrolled in the study as controls. Parameters of 24 hour-heart rate variability influenced by sympathetic (mean N-N interval, low frequency and total power) or parasympathetic system (square root of the mean squared differences of successive $\mathrm{N}-\mathrm{N}$ intervals, and high frequency) and low/high frequency ratio (balance between sympathetic and parasympathetic system) were compared between patients and controls, also between patients with storage and voiding symptom-predominant symptoms.

Results: There was no statistically significant difference between patients and controls in 24-hour-heart rate variability parameters influencing autonomic nervous system. There was also no significant difference between patients with storage and voiding symptom-predominant lower urinary tract symptoms.

Conclusions: Preclinic and limited number of clinic studies revealed the role of sympathetic system in benign prostate hyperplasia. To our knowledge, there is no study determining autonomic nervous system by 24-hour-heart rate variability. Normal systemic autonomic nervous system may raise different responses in lower urinary tract. In order to determine the role of autonomic nervous system in benign prostate hyperplasia; regional status of autonomic nervous system in the lower urinary tract must be considered rather than systemic autonomic nervous system.
UP-063

Randomized Double-blind Placebo Controlled Trial of Intradetrusor Injections of Botulinum Toxin for the Treatment of Refractory Overactive Bladder Secondary to Benign Prostatic Hyperplasia

Elterman, Dean; Lee, Daniel; Sheth, Seema; Chughtai, Bilal; Lee, Richard; Kaplan, Steven; Te, Alexis

Weill Medical College of Cornell University, New York, NY, United States Introduction and Objectives: Options for idiopathic overactive bladder $(\mathrm{OAB})$ refractory to medical and surgical management are limited. We assessed the efficacy of botulinum toxin $A$ (BoNT, Botox ${ }^{\circledR}$, Allergan, Inc., Irvine, $\mathrm{CA}$ ) in patients with refractory $\mathrm{OAB}$ secondary to benign prostatic hyperplasia (BPH).

Methods: This was a phase III, multicentre, randomized, double-blinded study conducted in 2 institutions. The following data represent the results from a single institution. Inclusion criteria included patients with $O A B$ secondary to $\mathrm{BPH}$, refractory to anticholinergic medication and persistent after surgical intervention to relieve obstruction, with an International Prostate Symptom Score (IPSS) $>12$. Patients were randomized in 1:1 fashion to either 20 injections of intradetrusor $10 \mathrm{U}$ BoNT vs. placebo. Six patients received BoNT vs. 7 placebo. Follow-up was performed at 1 week and then $1,3,6$, and 9 months. Voiding diaries, maximum flow rate (Qmax), post-void residuals (PVR), and IPSS scores were reviewed. The primary endpoint was frequency of micturition, with PVR, IPSS, and Qmax as secondary endpoints.

Results: Baseline characteristics were similar between the treatment groups, although body mass index was higher in the placebo group (32.7 vs. $26.8, p=0.02$, Table 1 ). The median age was 67 years, with a median IPSS of 20, Qmax of $13.5 \mathrm{~mL} / \mathrm{s}$, PVR of $49 \mathrm{cc}$, and 12 voids/day. Patients receiving BoNT demonstrated higher Qmax compared to placebo $(p<0.01$, Table 1$)$ at the 90 days follow-up. Urinary frequency, IPSS, and PVR were unchanged postoperatively in both groups.

Conclusions: Patients receiving BoNT showed a significantly higher Qmax than those receiving placebo. No significant changes in urinary frequency or symptom scores were seen however. Future studies with larger groups and longer follow-up time are needed to help characterize the utility of BoNT in treating $\mathrm{OAB}$ secondary to $\mathrm{BPH}$.

\section{UP-064}

Evaluation of Prostate Cancer Cells Responsiveness to an EGFR Targeting Combi-molecule

Poisson, Alexis; Delvoye, Nathalie; Le Page, Cécile; Péant, Benjamin; Mes-Masson, Anne-Marie; Saad, Fred

ICM/CHUM, Montreal, QC, Canada

Table 1. UP-063. Baseline characteristics

\begin{tabular}{|c|c|c|c|}
\hline & $\begin{array}{c}\text { Placebo } \\
(n=6)\end{array}$ & $\begin{array}{c}\text { Botulinum } \\
(n=7)\end{array}$ & $p$-value \\
\hline Age (years), mean $\pm S D$ & $66.7 \pm 9.6$ & $70 \pm 13.2$ & 0.62 \\
\hline BMI (lbs/in²), mean $\pm S D$ & $32.7 \pm 3.1$ & $26.8 \pm 2.3$ & 0.02 \\
\hline Baseline IPSS, median (IOR) & $21(9-24)$ & $16(13-23)$ & 0.72 \\
\hline $\begin{array}{l}\text { Baseline irritative IPSS } \\
\text { subdomain, median }\end{array}$ & $11.5(6-13)$ & $10(7-14)$ & 0.89 \\
\hline $\begin{array}{l}\text { Baseline } \mathrm{Qmax},(\mathrm{mL} / \mathrm{s}) \\
\text { mean } \pm \mathrm{SD}\end{array}$ & $16.4 \pm 6.5$ & $21.8 \pm 15.4$ & 0.44 \\
\hline $\begin{array}{l}\text { Baseline voided volume }(\mathrm{mL}) \\
\text { mean } \pm S D\end{array}$ & $214.3 \pm 93.1$ & $227.3 \pm 125.8$ & 0.84 \\
\hline PVR (mL), mean $\pm S D$ & $42.8 \pm 35.6$ & $38.3 \pm 37.9$ & 0.83 \\
\hline $\begin{array}{l}\text { Prostate volume }\left(\mathrm{cm}^{3}\right), \\
\text { mean } \pm \text { SD }\end{array}$ & $43.3 \pm 28.6$ & $87.1 \pm 40.1$ & 0.34 \\
\hline $\begin{array}{l}\text { Voiding frequency } \\
\text { preprocedure, median (IOR) }\end{array}$ & $12(10-12)$ & $9(8-12)$ & 0.28 \\
\hline
\end{tabular}


Introduction and Objectives: Chemotherapies currently used against advanced prostate cancer (PCa) often lead to chemoresistances, which greatly reduce treatment options and patient survival. New therapeutic strategies are essential to broaden the spectrum of remission-oriented chemotherapies while reducing adverse effects. ZR2003, a combi-molecule, contains two active chemical groups. Its quinazoline core (as in gefitinib) is designed to target the EGF Receptor (EGFR) and its hemi-mustard tail (analogous to half the mustard tail of chlorambucil) is known to inflict alkylation-meditated DNA damages. Recent data suggests that ZR2003 efficiently targets EGFR. As PCa progression is often associated with EGFR family of receptors, we hypothesized that ZR2003 could target PCa cells and induce an apoptotic cascade. Our goal was to determine the cytotoxic activity of ZR2003 in PCa cell lines.

Methods: The expression of EGFR and Her2 was determined in four PCa cell lines (LNCaP, 22Rv1, DU145 and PC3) by Western blot. The metabolic activity of DU145 following different ZR2003 doses was then evaluated using a WST-1 assay. The percentage of apoptotic ZR2003-treated cells was evaluated by FACS analysis of Annexin-V/7-AAD staining.

Results: Only DU145 cells overexpress the receptor EGFR. Her2 is highly expressed in LNCaP, 22Rv1 and DU145 cells while very low levels were observed in PC3 cells. DU145 cells treated with 6,5 $\mu \mathrm{M}$ ZR2003 concentration for $48 \mathrm{~h}$ induced a $50 \%$ decrease of metabolic activity. Moreover more than $35 \%$ of treated cells were apoptotic.

Conclusions: ZR2003 is cytotoxic to DU145 cells expressing high levels of EGFR and Her2. It greatly inhibits metabolic activity after exposure to the drug. The drug also induces a potent apoptotic signal in the same conditions. This therapeutic modality continues to be tested in the preclinical setting and may eventually be useful in a subset of castration resistant PCa patients in the future.

\section{UP-065}

Dynamic MRI Urethrography: Fluid Turbulence in a Urethral Stricture Model

Moles, Jonathan; Thornhill, Rebecca; Shabana, Wael; Saltel, Eric

The Ottawa Hospital, Ottawa, ON, Canada

Introduction and Objectives: Spongiofibrosis is an important factor in the pathophysiology of urethral stricture disease and may contribute to the proximal propagation of stenosis. A potential contributing factor to spongiofibrosis is urine extravasation from turbulence and high pressure voiding due to obstruction. Dynamic magnetic resonance $(M R)$ imaging has been used to evaluate fluid turbulence in the vascular surgery literature but its application to urethral strictures has not been assessed. Our goal is to explore the potential of dynamic MRI urethrography to quantify the obstruction and fluid turbulence in the urethra using a phantom model. Methods: A phantom was built consisting of two parallel 25F tubes with an $8 \mathrm{~F}$ stenosis in one tube. Each tube was connected to a saline and Gadolinium mixture and pressurized for a flow rate of $25 \mathrm{ml} / \mathrm{s}$. MRI was performed on a 3T (Siemens Tim Trio) and acquired images were Dynamic T1 weighted. Axial and sagittal views were acquired before, during and after the initiation of flow. The experiment was repeated 7 times. Regions of interest (ROI) were placed at the stricture, as well as directly proximal and distal. ROls were evaluated on the control tubing for reference and flow signals were compared.

Results: There was a strong visible signal drop across the stenosis in the experimental tube when compared to the control. The difference in signal intensity between locations proximal and distal to the stenosis was $74.36+/-2.58 \%$ of the prestenosis value in the experimental versus $94.22+/-4.23 \%$ in the control tube. Paired $t$ test showed $p=0.0004$, $\mathrm{t}=8.348,2$ tails.

Conclusions: Dynamic MRI does have utility in quantifying the degree of obstruction and turbulence in a urethral stricture phantom. Patient evaluation with dynamic MRI urethrography may provide both physiological and anatomical assessments. This may further clarify the relationship between urethral urine turbulence and spongiofibrosis in the propagation of urethral strictures.

\section{UP-066}

\section{Spicing up Radiation Treatment for Prostate Cancer}

Venier, Natalie ${ }^{1}$; Colquhoun, Alexandra ${ }^{1}$; Loblaw, Andrew D. ${ }^{1}$; Fleshner, Neil$^{2}$; Klotz, Laurence'; Venkateswaran, Vasundara

${ }^{1}$ Sunnybrook Health Sciences Centre, Toronto, ON, Canada; ${ }^{2}$ Princess Margaret Hospital, University Health Network, Toronto, ON, Canada Introduction and Objectives: Radio-sensitizing agents sensitize cells to the lethal effects of ionizing radiation (IR). This permits use of lower doses of radiation to achieve equivalent cancer control thereby minimizing adverse effects to normal tissues. Given their lack of toxicity compounds occurring naturally in the diet make ideal potential radio-sensitizing agents. Capsaicin is the active compound chilli peppers traditionally used to treat chronic pain syndromes; however, recently evidence describes its anti-carcinogenic potential using in vitro prostate cancer (PCa) models. We have demonstrated the radio-sensitizing capacity of in PCa cells in vitro. The objective of the present study is to assess the radio-sensitizing capacity in an in vivo model.

Methods: Athymic nude mice were inoculated with human PCa (LNCaP) cells. Once xenografts reach $100 \mathrm{~mm} 3$ forty animals will be randomized into 4 groups (15/group); control (no treatment), capsaicin alone, ionizing radiation (IR) alone and capsaicin and IR. Treatments were administered over a two-week time period. Capsaicin $(5 \mathrm{mg} / \mathrm{kg} / \mathrm{d})$ or vehicle was administered 3/week by gavage. RT was delivered to animals as one fraction (6 Gy). Tumors were measured thrice weekly. Tumors were fixed and stained for pathological analyses and IHC evaluation.

Results: There were no differences in the body weight of mice between groups. Two mice experienced mild to moderate inflammation of the stomach. No other toxicities were observed. Mice treated with capsaicin or IR alone had a significant reduction in tumor growth overtime $(p<0.001)$. Mice treated with capsaicin and IR capsaicin had a reduction in the tumor volume greater than either capsaicin alone $(p<0.001)$ or radiation alone $(p<0.03)$. We are currently investigating mechanism. Conclusions: These studies confirm the radio-sensitizing capacity of capsaicin in PCa xenograft mode. Ongoing studies are further delineating the mechanism of interaction of these treatment modalities.

\section{UP-067}

\section{Canadian Urology Resident Scholarly Performance}

Andrews, I. Matthew; Norman, Richard W.

Department of Urology, Dalhousie University, Halifax, NS, Canada

Introduction and Objectives: Research activities are an integral component of urology training programs. The objectives of the CanMEDS Scholar are often evaluated through successful research presentations and manuscript publication. Through comparison of scholarly performance of residents before residency with that achieved during residency, we aimed to elicit predictive factors for completion of research activities during residency.

Materials and Methods: Surveys were sent to 152 Canadian urology residents. Survey questions pertained to post-graduate year (PGY), education completed prior to residency and scholarly activity completed before/after the start of residency. The amount of protected research time, existence of a dedicated research rotation, existence of a structured research curriculum and pursuit of fellowship training were also examined.

Results: Surveys were completed by 42 residents from 11 programs. Only $26 \%$ of residents indicated their program included a structured research curriculum, $43 \%$ had protected research time and $38 \%$ had a dedicated research rotation. Of total residents, $45 \%$ had published $\geq 1$ manuscript during residency (mean $1.14+/-0.32$ ) and $43 \%$ had $\geq 1$ manuscript accepted/pending (mean $0.86+/-0.25$ ). Of residents $62 \%$ completed $\geq 1$ formal research presentation during residency, with $45 \%$ having presented a poster and $43 \%$ a podium. Only PGY significantly impacted the number of manuscripts published $(p<0.001)$ and formal research presentations $(p<0.001)$ during residency. Of the $86 \%$ of residents who intended on pursuing fellowship training, the mean number of publications and presentations during residency was $1.25+/-0.37$ and $2.25+/-0.54$, respectively.

Conclusions: PGY significantly impacted quantitative scholarly activity, but the numbers and types of presentations performed prior to residency, 
completion of an honors or graduate degree, and plans to pursue fellowship did not.

\section{UP-068}

\section{Virtual Problem-based Learning (PBL): A Needs Assessment} Examining Traditional versus Virtual Problem-based Learning

Mickelson, Jennifer $1 .{ }^{1}$; Lim, Rachel ${ }^{2}$; Amandeep, Ghuman ${ }^{2}$; Gourlay, William²; Masterson, John'; MacNeily, Andrew ${ }^{2}$

${ }^{1}$ University of British Columbia, Department of Urologic Sciences, Vancouver, BC, Canada; ${ }^{2}$ University of British Columbia, Vancouver, BC, Canada

Introduction and Objectives: The University of British Columbia has an increased need for flexibility in undergraduate medicine which can be created using virtual learning. This is a needs assessment of traditional problem-based learning (PBL) and the interest in virtual learning as part of PBL.

Methods: This is a qualitative and quantitative study utilizing focus groups and an electronic survey of 1 st and 2 nd year medical students at UBC. The data from the focus groups was analyzed using constant comparative analysis to identify themes. These themes were used to determined questions for the survey. Quantitative survey data was analyzed using a Chi-square test.

Results: Two focus groups of 1 st and 2 nd year students were undertaken to identify themes associated with traditional versus virtual PBL learning. The dominant theme from $1 \mathrm{st}$ and 2 nd year focus groups was concern over the loss of life-skills acquired during face-to-face PBL. The second most prevalent theme identified was the benefit of flexibility and convenience associated with virtual PBL learning. $73 \%$ of 1 st year and $17 \%$ of 2 nd year students responded to the electronic survey. $59 \%$ of 1 st year and $43 \%$ of 2 nd years felt that face-to-face interaction of PBL was "critically" or "strongly important" $(p<0.005) .29 \%$ of 1 st year and $61 \%$ of 2 nd year students wanted a hybrid of both virtual and traditional PBL $(p<0.005)$. $17 \%$ of 1 st year students and $36 \%$ of 2 nd year students felt that the addition of virtual PBL would be most beneficial during the 2 nd undergraduate year $(p<0.005) .66 \%$ of 1 st year students did not want virtual PBL as part of their undergraduate curriculum.

Conclusions: 1 st year students were more strongly guarded than 2 nd year students about using virtual PBL. Both groups felt it could be complementary in 2nd year. Respondents felt that a "hybrid" of a virtual and face-to-face experience later in 2nd year training would allow students to benefit from flexibility and convenience provided by virtual learning.

\section{UP-069}

\section{A National Survey of Chief Residents on the Communicator Role in Urology Residency: Are We Communicating the Message?} Roberts, Gregory; Fuoco, Michael; Beiko, Darren; Touma, Naji; Siemens, D. Robert

Queen's University, Urology, Kingston, ON, Canada

Introduction and Objectives: We sought to assess Urology residents' perceptions and attitudes toward the Communicator Role as defined by CanMEDS, and the current effectiveness of education aimed at this crucial competency.

Methods: An anonymous, cross-sectional, questionnaire administered to all final year Urology residents in Canada from two consecutive graduating years (2010-2011). A closed-ended 5-point Likert scale was used to assess familiarity with the Communicator Role and its importance to training and practice. Descriptive and correlative statistics were used and for ease of reporting, an agreement score was created for those responding with "strongly agree" and "agree".

Results: Response rate was $100 \%(\mathrm{~N}=58)$. Only awareness of communication as part of objectives, and ability to list all 7 roles were statistically different between years ( $p=0.01, p=0.03$ ). Only $45 \%$ could identify the correct number of CanMEDS roles, and only $19 \%$ could correctly list all seven roles. The vast majority was aware of the Communicator role, and most believed it to be important for practice. This is in stark contrast to perceived formal training. A minority (38\%) agreed that formal training or mentorship in communication was available at their institution, and only $38 \%$ felt that communication had been addressed during explicit sessions. Despite $84 \%$ of residents noting a significant mentor/role model to emulate with $93 \%$ aware that communication is part of their evaluations, only $48 \%$ believed that faculty frequently addressed communication during clinical learning experiences.

Conclusions: Despite knowledge and acceptance of the importance of the Communicator Role, there is a perceived lack of formal and informal training of this essential role of Urology residency. It would seem apparent that there is a need for a redoubling of efforts to ensure appropriate instruction and evaluation in our training programs.

\section{UP-070}

Is Online Script Concordance Test a Good CPD Tool to Induce Reflection on Controversial Issues in the Management of Prostate Cancer?

Bénard, François ${ }^{1}$; St-Germain, France ${ }^{2}$; Lamoureux, Cristine'; Saad, Fred ${ }^{3}$; Charlin, Bernard $^{1}$

${ }^{1}$ University of Montreal, Montreal, QC, Canada; ${ }^{2}$ Sanofi-Aventis Canada, Laval, QC, Canada; ${ }^{3}$ Centre hospitalier universitaire de I'Université de Montréal (CHUM), Montreal, QC, Canada

Introduction and Objectives: The Continuing professional development (CPD) programs usually available for urologists aim at the acquisition of new knowledge, including 'true of false' and 'multiple choice' questions. Few programs aim to induce reflexivity on practice, in context of uncertainly, where the answers are not 'black or white'. The purpose of this project is to design an innovative e-learning format that induces, through the use of script concordance tests (SCT), reflection on controversial issues in clinical practice. Program design and delivery.

Methods: Using needs assessment results, a committee identified the most common controversies associated with prostate cancer. The program comprises 7 cases, each followed by a set of questions assessing the impact of additional information on specific clinical decisions. A panel of experts provided answers for the questions and shared educational material to support their positions, which were incorporated into the web platform. Participants complete the program individually online. They answer the SCT questions (pre-test), compare their choices (scores) with those from the experts, read the enduring educational material, comment on experts' opinion in the forum, answer the SCT questions a second time (post-test), and then complete an evaluation form.

Results: 19 Canadian urologists validated the program. $78 \%(n=15)$ demonstrated a 'high interest' for the program content and format, $10 \%(n=2)$ indicated a 'medium' interest. $89 \%(n=17)$ confirmed their intention to continue the program, as they will receive a new case monthly through the web platform. Factors motivating participation are: experts 'opinion, excellent literatures review on the platform, program induces reflection on own practice, cases are clinically relevant.

Conclusions: The Script Concordance Test, for CPD was very well received by a group of Canadian urologists. The next step consists of measuring the clinical impact of this SCT program.

\section{UP-071}

A Systematic Review on the Effect of Lysine Analogs on Blood Loss and Transfusion Requirements during Pelvic Surgery Punjani, Nahid'; Kokolo, Madzouka B. ${ }^{2}$; Fergusson, Dean²; Cagiannos, Ilias $^{3}$; Knoll, Gregory ${ }^{4}$; Momoli, Franco ${ }^{2}$; Morash, Christopher G. ${ }^{3}$; Lavallée, Luke T. ${ }^{3}$; Tinmouth, Alan ${ }^{5}$; Breau, Rodney $\mathrm{H}^{3}{ }^{3}$

${ }^{1}$ University of Ottawa, Ottawa, ON, Canada; ${ }^{2}$ Ottawa Hospital Research Institute, Ottawa, ON, Canada; ${ }^{3}$ University of Ottawa, Division of Urology, Ottawa, ON, Canada; ${ }^{4}$ University of Ottawa, Division of Nephrology, Ottawa, ON, Canada; ${ }^{5}$ University of Ottawa, Division of Clinical Hematology, Ottawa, ON, Canada

Introduction and Objectives: Major pelvic surgery is frequently associated with blood loss requiring transfusion. Lysine analogs have been shown to decrease blood loss during cardiac and orthopedic surgery. The purpose of this systematic review was to detail and summarize trials that have evaluated the effect of lysine analogs during pelvic surgery.

Methods: A systematic and comprehensive search strategy was used. Studies were eligible if they reported perioperative administration of a lysine analog (tranexamic acid, aminocaproic acid, or aminomethylben- 
zoic acid) for a surgical procedure involving the pelvic region (digestive, urogenital, musculoskeletal, neurovascular, or integumentary). Studies evaluating trauma surgeries were excluded. Data extraction was duplicated by two trained reviewers on standardized electronic forms.

Results: The search strategy identified 2,660 citations that were screened (titles/abstracts) for eligibility. We obtained full-text versions of 68, of which, 30 were eligible. These 30 reported 34 trials that enrolled a total of 3,345 patients. Studies were published between 1961 and 2009 (half before 1971), and enrolled 8 to 515 patients (mean=96). Twenty-two studies tested aminocaproic acid, none tested aminomethylbenzoic acid, and 12 tested tranexamic acid. Across pelvic surgeries, lysine analogs decreased the risk of receiving at least one unit of blood by $31 \%$ (RR: $0.69,95 \% \mathrm{Cl} 0.47$ to 1.00 ). The pooled estimated decreased blood loss due to lysine analogs was $65.1 \mathrm{ml}(95 \% \mathrm{Cl}$ : 29.5 to 100.6).

Conclusions: Despite significant evidence supporting the use of lysine analogs in cardiac and orthopedic surgery, there are few studies exploring the benefits and harms associated with lysine analogs during pelvic surgery. Trials that have been conducted in pelvic surgery do suggest a benefit, but are inadequate in quality and quantity to inform clinical practice. Clinical trials on the effect of lysine analogs during major pelvic surgery are warranted.

\section{UP-072}

Antibiotic Prophylaxis Prescribing Patterns for Trans-urethral Resection of Prostate: a Need for Canadian Guidelines?

Lawson, Keith'; ; Vicas, Ingrid²; Hofmeister, Marianna²; Lockyer, Jocelyn²; Carlson, Kevin ${ }^{1}$

${ }^{1}$ Division of Urology, University of Calgary, Calgary, $A B$, Canada; ${ }^{2}$ Physician Learning Program, University of Calgary, Calgary, AB, Canada Introduction and Objectives: We set out to determine practice patterns amongst Urologists with respect to antibiotic prophylaxis for trans-urethral resection of prostate (TURP) and greenlight laser photovapourization of prostate (PVP). Preliminary analysis of a large ongoing study was conducted to determine its feasibility.

Methods: A retrospective chart review of 68 patients undergoing TURP in November 2009 in Calgary was completed. Antimicrobial prescribing patterns (preoperative, perioperative, postoperative) of 14 Urologists were recorded. Antibiotics administered 3 hours prior to and 24 hours post procedure were included. Additional data included patient demographics, presence of an indwelling catheter and pre/postoperative urine culture results. Patients were further categorized as undergoing elective versus emergent surgery. The primary outcome was compliance to the AUA's published "Best Practice Statement on Urologic Surgery Antimicrobial Prophylaxis" for TURP.

Results: Of the 68 patients reviewed 18 had preoperative indwelling catheters and 12 underwent TURP on an emergent basis. In total, 7 preoperative antimicrobial prescribing patterns were identified, which varied based on individual surgeon and the nature of the surgical case. Overall compliance to AUA best practice statement was $90 \%$. Poor compliance was noted for patients with indwelling urinary catheters (17\%).

Conclusions: Antimicrobial prescribing patterns prior to TURP vary considerably at our institution, with significant cost-benefit implications. Formal guidelines may significantly improve the rates of postoperative urinary tract infections as compliance with AUA best practice statement was lower than anticipated. Our pilot project suggests larger scale studies are warranted to determine optimal antimicrobial prophylaxis strategies prior to TURP and PVP.

\section{UP-073}

En Bloc Vascular Stapling of Renal Hilum during Laparoscopic Nephrectomy

McKay, Jeffrey ${ }^{1}$; Goodman, Saul'2; Neville, Christopher ${ }^{3}$; Cripps, Stephanie ${ }^{4}$; Perks, Alexandra ${ }^{4}$

${ }^{1}$ University of British Columbia, Vancouver, BC, Canada; ${ }^{2}$ Fraser Health Authority, Chilliwack, BC, Canada; ${ }^{3}$ Fraser Health Authority, Abbotsford, BC, Canada; ${ }^{4}$ Fraser Health Authority, Langley, BC, Canada

Introduction and Objectives: Classically, it is recommended to separately perform the ligation and division of renal hilar vessels during nephrectomy to prevent arteriovenous fistula formation. Although described rarely following open nephrectomy, an arteriovenous fistula has never been reported following laparoscopic nephrectomy. In this study, we demonstrate the safety and feasibility of en bloc stapling of the renal hilum as a standard approach to laparoscopic nephrectomy and nephroureterectomy in the community setting.

Methods: This retrospective review included all laparoscopic nephrectomy and nephroureterectomy cases performed by 4 surgeons from Sept 2007 to Aug 2011. Each case involved two urologic surgeons in a mentorship capacity. Surgical outcomes were compared between the en bloc stapling and individual vessel ligation groups. A chart review was performed using the integrated health authority online charting system, which includes all subsequent emergency room visits and hospital-based imaging in the Fraser Valley.

Results: 70 cases were included. $47(67 \%)$ cases received en bloc vascular stapling while $18(26 \%)$ received individual vessel ligation. 5 (7\%) cases required open conversion. Operative times were significantly shorter in the en bloc vs. individual vessel ligation group (140 vs. 225 minutes; $p<0.05$, respectively). No bleeding complications, open conversions, emergency room visits, or postoperative images demonstrated complications related to the technique used for renal hilar control.

Conclusions: Laparoscopic control of the renal hilum is one of the more difficult tasks to become proficient with when learning laparoscopic nephrectomy. En bloc stapling of the renal hilum provides a safe and efficient approach to laparoscopic nephrectomy and encourages urologists to incorporate laparoscopy into standard practice. Few cases of arteriovenous fistula have been reported after open nephrectomy but are rare and not due to vascular stapling devices.

\section{UP-074}

Evaluation of Autonomic Nervous System Activity by 24 Hour Holter Monitorization in Women with Idiopathic Overactive Bladder

Zorba, Orhan Ünal; Uzun, Hakki; Cicek, Yuksel; Kirbas, Serkan; Bostan, Mehmet

Rize University, Rize Medical Faculty, Rize, Turkey

Introduction and Objectives: To analyze autonomic nervous system (ANS) activity in patients with idiopathic overactive bladder (iOAB); we compared the results of 24 hour- heart variability (HRV) between iOAB patients and healthy controls.

Methods: A total of 43 women with iOAB (mean age: $47.6 \pm 13.5$ years) and 23 healthy controls (mean age: $44.6 \pm 2.5$ years) were enrolled in the study. Parameters of HRV influenced by sympathetic (mean N-N interval [SDNN], low frequency (LF) and total power [TP]) or parasympathetic system (square root of the mean squared differences of successive N-N intervals [RMSSD], and high frequency [HF]) and LF/HF ratio (which represents the balance between sympathetic and parasympathetic system) were compared between patients and controls.

Results: All the patients' laboratory results were within normal limits, and no significant differences were found between patients and controls as for age, body weight, body mass index, menopausal status. On time domain analysis, SDNN, RMSSD and mean heart rate of the patients with iOAB were higher than the control group without statistically significant difference. On frequency domain analysis, HF was higher, while TP, LF and LF/ HF ratio were lower in the $\mathrm{BOAB}$ group without statistical significance. Conclusions: To our knowledge, there is no study determining ANS functions by 24 hour - HRV recording in women with iOAB, and we didn't find any difference between control subjects and patients with $\mathrm{BOAB}$ as for HRV variability. IOAB patients may have a global ANS disturbance, as a response to unknown etiology rather than an etiologic factor for $\mathrm{iOAB}$. 


\section{UP-075}

\section{Seasonal Variation in Urological Pelvic Pain Syndromes: Seven Years of Google ${ }^{\mathrm{TM}}$ Search Trends}

Roberts, Gregory'; Williamson, Tyler²; Teichman, Joel M.H. ${ }^{3}$; Nickel, J. Curtis ${ }^{1}$

${ }^{1}$ Queen's University, Kingston, ON, Canada; ${ }^{2}$ Centre for Studies in Primary Care, Kingston, ON, Canada; ${ }^{3}$ Department of Urological Sciences, University of British Columbia, Vancouver, BC, Canada

Introduction and Objectives: Anecdotal evidence suggests a seasonal variation in flares in urologic chronic pelvic pain syndromes. Google search history has been validated for confirming seasonal correlations in viral and cold outbreaks, lyme disease peaks, and kidney stone frequency trends. We used a similar methodology to assess the hypothesis that there is indeed a seasonal variability in symptoms of interstitial cystitis (IC) and prostatitis.

Methods: The search term "interstitial cystitis" (IC) and "prostatitis" were each evaluated though Google Insights for Search in Canada over the period of 2005-2011. New York State was used as a control for Canadian data. Interval scale data obtained from the tool was then compiled and analyzed using a generalized linear model assuming a Gaussian distribution and an identity link. Mean Google trend scores were compared across the various groups using a t-test for unpaired data.

Results: Longitudinal assessment of IC and prostatitis over the 7 years revealed no obvious pattern. In average monthly searches, no significant year difference was appreciated for either IC $(p=0.643)$ or prostatitis $(p=0.892)$. No significant difference between months, or seasons was demonstrated for IC ( $p=0.285$ and 0.937 respectively) and prostatitis $(p=0.120$ and 0.446 respectively). The New York data similarly showed no significant seasonal differences $(p=0.512)$.

Conclusions: There is no seasonal variation in Internet search patterns for urological chronic pelvic pain syndromes. We were unable to prove that that IC and prostatitis symptoms are typically seasonal.

\section{UP-076}

Sub-inhibitory Antibiotic Concentrations Negatively Affects Both the Uropathogen Staphylococcus Saprophyticus and Host Immune Responses

Fahmy, Nader; Fuller, Andrew; Goneau, Lee; MacDonald, Kyle; Erdeljan, Petar; Bathini, Varunkumar; Razvi, Hassan; Cadieux, Peter

The University of Western Ontario, London, ON, Canada

Introduction and Objectives: Staphylococcus saprophyticus is the most common Gram-positive uropathogen and is only second to E coli as the most frequent cause of uncomplicated and recurrent urinary tract infections (UTI) in young females. While the effects of sub-inhibitory concentrations of antimicrobials on multiple organisms have been studied, still little is known regarding $\mathrm{S}$. saprophyticus response. This may represent an important oversight since transiently low antimicrobial concentrations are present in patients undergoing prophylactic therapy for recurrent UTI. Methods: The effects of sub-Minimum Inhibitory Concentrations (MIC) of ciprofloxacin, ampicillin and gentamicin on S. saprophyticus attachment to glass microscope slides were tested. Using sub-MIC of ciprofloxacin, the adherence to ureteral stent material and T24 bladder cells, as well as pro-inflammatory cytokine expression in bladder cells were assessed. The ability of sub-MIC antibiotics to enhance survival against subsequent bactericidal challenge was measured.

Results: Adherence to microscope slides, ureteral stent material and T24 bladder cell monolayers were significantly increased in the presence of sub-MIC levels of antibiotics. While S. saprophyticus challenge of T24 bladder cell monolayers significantly upregulated both IL-6 and IL-8 expression, the addition of sub-MIC ciprofloxacin abrogated these effects. Pre-treatment of S. saprophyticus with sub-MIC antibiotics improved its ability to survive subsequent treatment with typically lethal concentrations of the same agent.

Conclusions: Exposure to sub-MIC antibiotics increases S. saprophyticus adherence to both abiotic and biotic surfaces. Low levels of ciprofloxacin downregulate pro-inflammatory cytokine secretion in bladder cells. These changes may improve its ability to colonize the urinary tract, highlighting the need for clinicians to consider the impact of sub-inhibitory concentrations of antimicrobials when treating recurrent UTI.

\section{UP-077}

First Inflatable Penile Prosthesis Infections and a Proposed Survey for Post-implant Monitoring

Narayanan, Ramkishen; Aliotta, Jessica; Leung, Tony; Duff, Michael; Barlog, Kevin; Chevli, K. Kent

University at Buffalo School of Medicine and Biomedical Sciences, Buffalo, NY, United States

Introduction and Objectives: We report on virgin inflatable penile prosthesis (IPP) infections and propose a follow-up survey for monitoring post-implant infection.

Methods: A single centre retrospective review of 149 men with first IPPs implanted for impotence from 1998-2011. Patient, device and operative factors were analyzed (Mann-Whitney $U$ test for continuous and Fisher's exact test for categorical variables) for association with IPP infection.

Results: A total of nine (6.0\%) first implants had culture-confirmed infection at revision: 6 were removed and 3 were salvaged at the initial revision. Median time to infected IPP revision was 3.1 months (range $0.7-41.8$ mos.), compared to 20.2 mos. (range 4.5-95 mos.) for mechanical failure, $p=0.004$. 1 salvaged man complained only of genital pain (no clinically apparent infection). At 1 hospital, mean initial operative (OR) time for 4 IPPs that developed infection was $123.0 \pm 32.0$ minutes, compared to $86.7 \pm 11.1$ mins for 36 uninfected prostheses. Age, obesity, diabetes, surgical approach (penoscrotal versus infrapubic), presence of a drain at the end of surgery, prosthesis or rear-tip extender length, and IPP antimicrobial coating did not affect infection-related revisions (each $p>0.1$ ). Conclusions: IPP infections present sooner for revision than mechanical failure, but the rate of salvage lags behind removal. Detecting persistent pain or other signs of subclinical infection may increase salvage. We, therefore, propose the Monitoring After Penile Prosthesis (MAPP) score: a repeatedly administered post-implant patient survey grading severity as 0-none, 1-mild, 2-moderate, 3-severe for each of erythema, induration, swelling, drainage, and pain with device use (activation/deflation/ejaculation); MAPP can be supplemented with the common 0-10 regional pain scale. We plan to prospectively evaluate how MAPP score trends affect revision rates. Shorter OR time may be key in reducing the incidence of IPP infection.

\section{UP-078}

How Many Semen Samples Are Required to Make the Diagnosis of Azoospermia?

Khambati, Aziz ${ }^{1}$; Jarvi, Keith²; Lo, Kirk C. ${ }^{2}$

${ }^{1}$ University of Toronto, Toronto, ON, Canada; ${ }^{2}$ Mount Sinai Hospital, Toronto, ON, Canada

Introduction and Objectives: Many guidelines now suggest that men are diagnosed as being azoospermic when no sperm is found in two sequential semen samples. Men have a significant degree of variability of sperm counts possibly due to variability in sperm production. This has led us to speculate that some men with non-obstructive azoospermia may also have variability in sperm production and hence might have enough sperm production to lead to sperm in the ejaculate. The study objective was to determine how many men who would typically be defined as being azoospermic had sperm in the ejaculate on subsequent testing.

Methods: A retrospective study was performed by using a database containing the semen analyses results of patients referred to our centre. Patients with a minimum of three semen samples, each within a space of 6 months, with the first two showing azoospermia were identified. Medical records were reviewed and patients with obstructive causes such as a vasectomy or a corrective procedure such as a varicocelectomy were excluded.

Results: In all, 120 men with a total of 420 semen analyses were included in the analysis. In men with two initial azoospermic samples, 27 out of $120(22.5 \%)$ had sperm on the third sample. Eight $(29.6 \%)$ of these patients had rare non-motile sperm, whereas the mean and median spermatozoa counts in the remaining men (19/27: $70.4 \%)$ were 0.54 and 0.4 million respectively. Four of $41(9.7 \%)$ men with three initial azoospermic 
samples had spermatozoa on the fourth sample. The average motility overall was $21.4 \%$. Finally, none of the 17 men who were azoospermic after four samples had any sperm identified in subsequent tests.

Conclusions: This study suggests that at least 3 semen samples should be examined before making the diagnosis of azoospermia. In addition, more than $20 \%$ of men who would have originally been diagnosed as azoospermic had enough sperm in the subsequent semen specimens to use in a program of intra-cytoplasmic sperm injection.

\section{UP-079}

Fertility of Patients with a History of Bilateral Cryptorchidism Treated: a Comparative Study about 120 Patients

Mallat, Faouzi; Hmida, Wissem; Hidoussi, Adnen; Slama, Adel; Jaidane, Mehdi; Ben Sorba, Nebil; Mosbah, Ali Faouzi

'Department of Urology, Sahloul, Sousse, Tunisia

Introduction and Objectives: Assessing fertility (sperm and the rate of surgical sperm collection) in a population of patients with a history of treated bilateral cryptorchidism.

Methods: Retrospective and comparative study made in a department of urology and obstetrics and gynecology department of 120 patients aged between 18 and 30 years divided into four groups: group I (30 patients with bilateral testicular lowering), group II (30 patients with unilateral testicular lowering) Group III (30 patients with untreated bilateral testicular ectopia), group IV (30 patients with untreated unilateral undescended testes). A semen analysis and a testicular sperm extraction (TESE) was performed. We studied the reduction of age before or after the age of ten. Then we studied the different parameters of semen analysis, and calculated the rate of positive surgical extraction of sperm according to these different. Then, we studied the rates of successful TESE according to these various characteristics.

Results: The mean age was 28.6 years. A great majority of the patients $(81.2 \%)$ has benefited of an orchidopexy before the age of 7 years, which does not seem to represent a factor of better forecast of surgical extraction of sperm cells. In the subgroup of the bilateral cryptorchidy, the rate of extraction was $66 \%$. In the subgroup of the one-sided cryptorchidy, it was $63 \%$. The sperm was altered in $78 \%$ of patients with a history of cryptorchidism.

Conclusions: For us, history of cryptorchidism is an etiology of relatively good prognosis for infertility, since the rate of TESE with positive sperm retrieval is $65 \%$. In our population, the subgroups of patients whose FSH is normal and/or whose testicular volume is higher than $10 \mathrm{~cm} 3$ are those whose forecast is still better, because the rate of TESE with positive sperm retrieval is $75 \%$.

\section{UP-080}

\section{Spectrum of Ureteropelvic Junction Obstruction (UPJO) Morphological Characteristics from Infancy to Adulthood and Implications in Surgical Management}

Romao, Rodrigo L.P.'; Foell, Kirsten²; Figueroa, Victor H. ${ }^{1}$; Lorenzo, Armando'; Farhat, Walid'; Pace, Kenneth'²; Bagli, Darius'; Koyle, Martin'; Honey, R. John D'A. ${ }^{2}$; Pippi Salle, Joao L. ${ }^{1}$

${ }^{1}$ The Hospital for Sick Children, University of Toronto, Toronto, ON, Canada; ${ }^{2}$ St. Michael's Hospital, University of Toronto, Toronto, ON, Canada

Introduction and Objectives: UPJO is prevalent in children and adults however mode of presentation and anatomical features can vary with age. We sought to compare morphological aspects of UPJO in different age groups and possible implications in management.

Methods: Retrospective review of patients undergoing dismembered pyeloplasty from 2005-2011 in 2 centres (1 pediatric and 1 adult). Redo cases, secondary UPJO and patients without a retrograde pyelogram (RPG) were excluded. Data collected: age, side, length of the stenotic segment (LSS) and presence of crossing vessel (CV). LSS was determined by a ratio (LSSR) measured in pixels on the RPG: LSSR= length of the obstructed segment/height of a lumbar vertebrae taken perpendicular to the midportion of its body. Patients were divided in 4 age groups: 1) 0-5, 2) 6-10, 3) $11-18$ and 4) $>18$ years old.
Results: Age range was 1 month -79 years for the entire cohort (mean 42 months and 41.2 years for children and adults, respectively). 90 children $(<18$ years old) and 67 adults were included. Obstruction was left-sided in $74 \%$ of the pediatric and $43 \%$ of the adult group. There was no difference in the incidence of CV across groups 2-4(around 40\%); group 1 had a significantly lower CV rate $(10 \%)$. LSSR was significantly longer in group $1(0.6)$ with a progressive substantial decrease being observed throughout groups 2 and $3(0.33,0.38)$ and into adulthood $(0.1)$. Only in group 1 the presence of CV was associated with a significantly lower LSSR $(p=0.04)$. Conclusions: UPJO has distinct features with progression of age. Young children usually have intrinsic, longer segments of obstruction and the rare occurrence of $\mathrm{CV}$ is associated with a shorter length of obstruction. In older children and adults, the length of the obstruction is progressively shorter and not impacted by the higher incidence of CV. Our data support routine RPG as beneficial in the surgical planning for infants and young children and challenge its use in older children and adults.

\section{UP-081}

The Challenge and Morbidity of Intermittent Ureteropelvic Junction Obstruction (IUPJO) in Children

Romao, Rodrigo L.P.; Figueroa, Victor H.; Pippi Salle, Joao L.; El-Naeema, Aziza; Lorenzo, Armando; Bagli, Darius; Farhat, Walid; Koyle, Martin The Hospital for Sick Children, University of Toronto, Toronto, ON, Canada

Introduction and Objectives: IUPJO represents a diagnostic challenge. Our goal was to evaluate potential pitfalls in the diagnostic process and the morbidity associated with IUPJO.

Methods: Retrospective review of children undergoing pyeloplasty (PP) in a 10-year period. Only patients presenting with intermittent abdominal pain and absence of or grade $1 \mathrm{SFU}$ hydronephrosis $(\mathrm{HN})$ were included. Demographics, duration of pain, yield of imaging tests, surgical procedures, resolution of symptoms postoperatively were collected.

Results: $18 / 455$ patients having PP $(4 \%)$ were included. Mean age was 10 years (6-15) with male (78\%) and left-sided UPJO (67\%) preponderance. Pain location was flank in $15 / 18(84 \%)$ patients and mean duration was 2 years ( 3 months-7 years). Nausea/vomiting and exacerbation by fluid intake were present in $50 \%$ and $33 \%$, respectively. Mean number of imaging studies (US, CT scan, nuclear scans, IVP and MRU) before PP was 5.5 (2-12). Total number of nuclear scans was 26 , but only $5(19 \%)$ were abnormal. Imaging during pain had the best diagnostic yield: US and CT revealed severe $\mathrm{HN}$ in $6 / 7$ and $2 / 2$ patients, respectively. In 9/18 (50\%) patients, a "stress" test with a fluid load and furosemide mimicked symptoms without an abnormal imaging result in $7 / 9$. One patient underwent laparoscopic appendectomy with persistence of pain and 3 patients had a stent insertion before PP (pain relief in 2). 6/18(33\%) patients had crossing vessels. Dismembered PP was performed in 17 patients and laparoscopic angiopexy in 1 ; symptomatic resolution was achieved in 15/18 (83\%).

Conclusions: IUPJO was more common in males and usually presented with flank pain associated with nausea/vomiting. Diagnosis is challenging and ideally achieved with imaging performed at the time of the pain episode. "Stress" tests may be helpful for mimicking symptoms, but not necessarily will translate into abnormal results. IUPJO requires a comprehensive approach with a realistic expectation of improvement after PP. 


\section{UP-082}

\section{Role of Magnetic Resonance Urography in Girls with Urinary Incontinence: a Single Centre Experience}

Figueroa, Victor H.; Chavhan, Govind; Romao, Rodrigo L.P.; Lee, Justin K.; Pippi Salle, Joao L.; Bagli, Darius; Koyle, Martin; Lorenzo, Armando; Farhat, Walid

The Hospital for Sick Children, Toronto, ON, Canada

Introduction and Objectives: Ectopic ureters (EU) associated with continuous urinary incontinence are often difficult to diagnose despite routine imaging modalities. However, superior anatomic delineation provided by magnetic resonance urography (MRU) may be useful in assessing these patients. This study aims to determine the utility of MRU in the evaluation of EU.

Methods: Between November 2007 and July 2011, 17 females who failed different therapeutic intervention for incontinence underwent MRU to rule out a EU, using a Philips 1.5T and 3T MRI scanners. All patients had prior imaging studies for work up (17/17 Renal ultrasound, 3/17 VCUG, 10/17 Renal scan). The medical records were retrospectively reviewed and MRU findings re-examined by a single pediatric radiologist prior to correlation with intraoperative surgical findings.

Results: Mean age at the time of the MRU was 8.3 years (4-14). Out of 17 patients, $9(53 \%)$ had findings of lower urinary tract anomaly (LUTA) based on MRU. Of the remaining 8 (47\%) patients, MRU was either normal or showed duplex kidneys with orthotopic ureters (OU). Of 9 cases with LUTA, MRU suggested the presence of EU in all patients but one case that was diagnosed with persistence of uro-genital sinus and underwent surgical correction. In the remaining 8 , the decision to proceed with surgery was made. One child is awaiting surgery. Of those who underwent surgical intervention, 5 of $7(71.4 \%)$ were confirmed to have EU intraoperatively and underwent surgical correction. Two patients $(28.6 \%)$ had complete duplication of their collecting system with OU confirmed by cystoscopy and retrograde pyelogram.

Conclusions: In girls suspected of having ectopic ureters and when routine imaging modalities are indeterminate, MRU could be beneficial in delineating urinary tract anomalies. Further refinement of the MRU technology and analysis will be needed to enhance its sensitivity in depicting lower urinary tract anatomy.

\section{UP-083}

Peristeen Anal Irrigation as a Substitute of Mace Procedure in Children Who Are in Need of Reconstructive Bladder Surgery Alhazmi, Hamdan; Alenezi, Husain; Salem, Mahmoud; Fouda Neel, Khalid

King Saud University, Riyadh, Saudi Arabia

Introduction and Objectives: To evaluate the efficacy of Peristeen ${ }^{\circledR}$ Transanal irrigation (TI) system as a substitute of The MACE procedure in children who need reconstructive bladder surgery.

Methods: We prospectively evaluated children with neuropathic bladder and bowel dysfunction who were planned for reconstructive bladder surgery and MACE procedure. All patients were started on Peristeen $\mathbb{R}$ TI system at least 3 months before surgery to assess their response. The patient's bowel function, the frequency of using the system, patients and parents satisfaction and diaper independency were evaluated.

Results: Between 2007 and 2010, thirteen patients were included in our evaluation, 9 females and 4 males. The mean age of the group was 8.6 years (range $4-15$ years). Ten patients $(76.9 \%)$ showed complete dryness from stools. Of them, six children $(60 \%)$ were able to be diapers free, while 3 patients continued wearing diapers due to fear of soiling and 1 patient due to urinary incontinence. All ten children and their parents were satisfied by using Peristeen $囚$. They underwent reconstructive bladder surgery and were continued on Peristeen ${ }^{\circledR}$ with the same results postoperatively. All of the ten patients were using Peristeen $₫$ twice weekly. Three

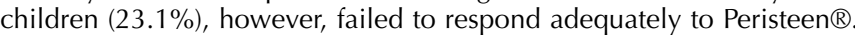
Two of them required concomitant MACE procedure with reconstructive bladder surgery, while the third patient and family refused any surgical options and continued on pharmacological management.

Conclusions: Our initial results suggest Peristeen $₫$ TI system to be a successful conservative substitute of MACE procedure in children who require reconstructive bladder surgery.

\section{UP-084}

Hemorrhagic Cystitis Secondary to BK Virus: A Case Report of Management with Intravesical Cidofovir in the Pediatric Patient and Review of Literature

Bidnur, Samir; Wu, Christopher; Rassekh, Rod; Mickelson, Jennifer J.; MacNeily, Andrew

University of British Columbia, Vancouver, BC, Canada

Introduction and Objectives: Cidofovir is a nucleoside analogue that has gained popularity in recent years for treatment of viral induced severe late hemorrhagic cystitis (HC) following hematopoietic stem cell transplantation (HSCT) in the adult population. It is administered intravenously although its adverse effects including marked renal toxicity are well described. Viral HC refractory to supportive care and intravenous cidofovir represents a particular challenge to the physician.

Methods: Several case reports within the adult population have described the use of intravesical cidofovir in the management of refractory viral $\mathrm{HC}$ with successful outcomes; however no similar cases exist within the pediatric population in English literature. Here, we describe the use of intravesical cidofovir in a pediatric patient with severe refractory BK virus $\mathrm{HC}$, several months post HSCT, with significant improvement of lower urinary tract symptoms and hematuria.

Results: The patient received a total of two instillations of cidofovir $(5 \mathrm{~mL} /$ $\mathrm{kg}$ ) in $60 \mathrm{~mL}$ normal saline on day +240 and +247 post HSCT.

Conclusions: Intravesical cidofovir may represent an effective and welltolerated treatment in the management of refractory viral $\mathrm{HC}$ in both the adult and pediatric populations.

\section{UP-085}

The Use of EMLA Cream During Circumcision: A Prospective Study about Sixty Children

Mallat, Faouzi; Hmida, Wissem; Hidoussi, Adnen; Slama, Adel; Jaidane, Mehdi; Ben Sorba, Nebil; Mosbah, Ali Faouz

Department of Urology, Sahloul, Sousse, Tunisia

Introduction and Objectives: Showing the effectiveness of EMLA in this new indication.

Methods: A prospective study was designed to assess the quality of skin analgesia provided by the EMLA anesthetic cream during circumcision in 60 children. The children, aged 3 months to 11 years, and scheduled for circumcision, were allocated to two groups, those aged $\leq 5$ years ( 3 to 58 months, $n=32$ ), and those aged $>5$ years ( 60 to 130 months, $n=28$ ). Cream $(1.6 \pm 0.6 \mathrm{~g})$ was applied in the room like a thick layer on the skin area to be anesthetized (along the penile skin, the root of the penis until the foreskin and on the glens), and covered by a plaster closed.

Results: This method required $2.6 \pm 1.7 \mathrm{~min}$, and was considered very easy $(92 \%)$ or easy $(8 \%)$. Circumcision was performed $93 \pm 52$ min after application of the cream. Children aged $\leq 5$ years complained of a pain intensity of $7 \pm 1.2$ (CHEOPS scale, range 4-13) and, for those aged $>5$ years, $22 \pm 21$ on a visual analogue scale (range 0 to 100). Local adverse events occurred in six patients (pale skin, erythema, or both).

Conclusions: The coetaneous analgesia by EMLA cream during circumcision was measured, and statistical analysis of results shows the effectiveness of EMLA in this new indication.

\section{UP-086}

Glandular Amputation during Circumcision: A Review of 12 Cases Results of a New Technique for Glans Self-transplantation Mallat, Faouzi; Hmida, Wissem; Hidoussi, Adnen; Slama, Adel; Jaidane, Mehdi; Mosbah, Ali Faouzi

Department of Urology, Sahloul, Sousse, Tunisia

Introduction and Objectives: To assess the clinical features and describe a successful new surgical treatment of the glandular amputation during circumcision.

Patients and Methods: Retrospective study of 12 patients who suffered glandular amputation during circumcision. The medical charts of patients were studied. 7 patients brought with the cut segment of the glans, within a period of time less than 4 hours. Group 1: 4 patients underwent reimplantation after urethral anastomosis and anastomosis interesting the two 
parts of the glans and corpora cavernosa without achieving the shunts. Group 2: 3 patients had the same method but with the realization of several shunts every 4 hours for 72 hours. The other 5 patients had a tummy by performing a skin graft. All patients were seen every two months. Sensibility the glans and erection were studied.

Results: The mean age of these patients was 3.6 years, 7 patients $(58,3 \%)$ were presented with complete glandular amputation. The mean time to consultation was 2 hours (ranged from 30 minutes to 8 hours). The relocation of the glans was carried out immediately. The average duration of operation was $70 \mathrm{mn}$. The average length of hospital stay was 16 days. For the group 1, glandular reimplantation was unfortunately failed (necrosis of the gland was noted on the third day), two of them had narrowing of the meatus again treated by méatoplasty. A success was noted for all patients in Group 2, one patient will builds a stricture at the anastomosis area in the third month, treated by internal urethrotomy with a good evolution. Susceptibility of the glans and erection were preserved in group 2.

Conclusions: The new technique of reimplantation of the glans with the realization of a shunt is an effective technique for treatment of glandular amputation.

\section{UP-087 \\ Cystic Intratesticular Lesions in Pediatric Patients: a Retrospective Case Series}

Hoag, Nathan; Afshar, Kourosh; Youssef, David; Masterson, John; Murphy, James; MacNeily, Andrew

University of British Columbia, Vancouver, BC, Canada

Introduction and Objectives: Intratesticular cysts are a rare clinical entity in the pediatric population for which a paucity of data exists to guide optimal management. Traditionally, orchiectomy was performed, and more recently, testes sparing surgery has been recommended. We share our experience with the management of pediatric testicular cysts.

Methods: A retrospective review of all pediatric patients referred for intratesticular cysts was conducted at a single pediatric urology institution from 2002-2010. Charts were evaluated for patient demographics, diagnosis, and management.

Results: Seven patients were identified and included in this case series. Conclusions: All of the cystic lesions in our case series were benign with one undergoing complete resolution. The remainder became smaller and developed a solid component prompting surgery. The pre-pubertal findings of ITGCN in two of the seven patients raise a clinical dilemma regarding the natural history and optimal long-term management for these patients. Initial conservative observation is an option for the majority of pre-pubertal cystic testicular lesions until such time that testis sparing surgery is deemed technically feasible. Testes sparing surgery should be advocated in those patients undergoing surgical management.

\section{UP-088}

\section{Retrospective Review of Diagnosis of Testicular Torsion in Boys Presenting to Pediatric Emergency Department with Acute Scrotal Pain}

Liang, Teresa'; Metcalfe, Peter ${ }^{2}$; Sevcik, William²; Noga, Michelle ${ }^{2}$

${ }^{1}$ University of British Columbia, Burnaby, BC, Canada; ${ }^{2}$ University of Alberta, Edmonton, AB, Canada

Introduction and Objectives: Testicular torsion is a common acute condition in boys requiring prompt and accurate diagnosis. The objective was to evaluate ultrasound (US) accuracy, clinical predictors, and review urology care of boys presenting to the Stollery pediatric emergency department (ED) with acute scrotal pain.

Methods: Retrospective review of US, surgical and ED records for boys aged 1 month to 17 years, presenting with acute scrotum from 2008 to 2011, was performed. Age, demographics, clinical symptoms, physical findings, US and surgical techniques, findings and diagnoses were recorded.

Results: 342 patients presented to the ED with an acute scrotum and were diagnosed with: $35(10 \%)$ testicular torsion, 12 (3.5\%) possible torsion-detorsion, $3(0.9 \%)$ torsion of appendix testes, 135 (39\%) epididymo-orchitis, and 157 other. Diagnostic accuracy of US was $96 \%$ for torsion, using surgical diagnosis as gold standard. Six torsion patients went directly to the OR without an US. The US finding of heterogeneity of testes on ultrasound was not statistically associated with orchiectomy $(p=0.09)$. Mean time from ED to US and surgery for torsion patients was 159 and 303 minutes respectively. Twenty-four patients had salvageable testes $(68.6 \%)$. Mean time from symptoms onset until surgery between the non-salvageable and salvageable groups was not significantly different at $1714 \mathrm{~min}$ and $1127 \mathrm{~min}(p=0.36)$. Sudden-onset scrotal pain $(88 \%)$, abnormal position ( $86 \%$ ) and absent cremasteric reflex (91\%) were most prevalent in torsion patients.

Conclusions: Color Doppler US is accurate and sensitive for diagnosis of torsion in the setting of acute scrotum. Despite concerns with necrosis, a finding of heterogeneity was not associated with orchiectomy or atrophy. Salvage rate is consistent with previous publications, but time to surgery needs to be reduced.

\section{UP-089}

Penoscrotal Lymphedema: Sexuality, Fertility, Functional and Aesthetic Outcome of the Surgical Treatment - Our Experience about 14 Cases

Mallat, Faouzi; Hmida, Wissem; Hidoussi, Adnen; Slama, Adel; Jaidane, Mehdi; Ben Sorba, Nebil; Mosbah, Ali Faouzi

Department of Urology, Sahloul, Sousse, Tunisia

Introduction and Objectives: To evaluate the sexuality, the fertility, and aesthetic outcome of the surgical treatment of patients with penoscrotal lymphedema (PSL).

Methods: From January 2000 to December 2010, we treated 14 patients with PSL using reconstructive surgery. Patients underwent a testosterone dosage, testicular Doppler ultrasound, a semen analysis prior to reconstructive surgery. A testicular biopsy and a pathological examination of the excised tissue were analyzed. Sexuality was assessed by a routine questionnaire about Sexual function (Penetration, Partner satisfaction, Pain during erection) and by a score IIEF15.

Results: The mean patient age was 39 years (range 18-52). The median follow-up time was 64 months (range 12-120). The average duration of disease progression was 12.8 years (6-28 years). The disease was congenital in 5 patients and secondary in 9 . Association with lymphedema of the lower limbs was found in 7 patients. Preoperative semen analysis done in 9 patients has been altered more severe in case of congenital elephantiasis. After reconstruction, there was no significant improvement of semen analysis. The rate of testosterone was normal. Testicular biopsy and testicular ultrasound were not noted with particular aspects. All parameters of sexuality were disturbed before surgery, improved slightly after surgery, five patients remained impotent. Despite an improvement in the quality of life, all patients are dissatisfied with their sexuality.

Conclusions: Penoscrotal elephantiasis is a serious disease that destroys so deep sexuality and fertility. Reconstructive surgery is the only treatment that may correct the appearance but not a major effect on sexuality and fertility.

\section{UP-090}

Comparison of Apoptotic Gene Expression Profiles between Peyronie's Disease Plaque and Control Tunica Albuginea Zorba, Orhan Ünall'; Ozgon, Gulay²; Sirma, Sema²; Ozbek, Ugur²; Kadıoglu, Ates ${ }^{2}$

${ }^{1}$ Rize University, Rize Medical Faculty, Rize, Turkey; ${ }^{2}$ Istanbul University, Istanbul Medical Faculty, Istanbul, Turkey

Introduction and Objectives: The fibrotic plaques of Peyronie's disease (PD) and other localized fibrotic conditions have been considered to be the result of an abnormal wound healing process. Potential pathogenetic role of disorders in the regulation of apoptosis in abnormal wound healing may also play a role in the development of Peyronie's disease. To examine the phenomenon of apoptosis in Peyronie's disease, we quantified differential levels of expression of Fas, Fas Ligand, Bcl-2, p53, Caspase 3 and 8 which have major roles in apoptosis, gene expressions in Peyronie's plaque and control tunica albuginea (TA).

Methods: Eight Patients with PD undergoing surgical correction of the curvature had biopsy specimens removed from the Peyronie's plaques and normal TA. Messenger RNA (mRNA) expression was assessed in 
plaques and normal tunica by reverse transcriptase PCR. The levels of housekeeping gene " $\beta 2$ microglobulin" were used as internal control for normalization of RNA quantity and quality differences in all samples. Results: Apoptotic gene expressions were lower than housekeeping gene in half of the patients with normal TA and two thirds of the cases with plaques. The mRNA expressions in the plaque were not significantly different from the normal TA.

Conclusions: Lower expression of apoptotic gene relative to the housekeeping gene may cause the persistence of collagen producing cells, which were up-regulated due to unknown reasons and consequently plaque formation. Similar expression levels of apoptotic genes in both TA and Peyronie's plaques may be due to the generalized physiopathologic alterations in TA that lead to plaque formation at a vulnerable region subjected to recurrent traumas.

\section{UP-091}

Climacturia Post-prostatectomy Remains a Largely Unknown Clinical Entity in Contemporary Urologic Training Based on Results Obtained at a Resident Training Laboratory

Zappavigna, Christopher ${ }^{1}$; Christine, Brian²; Bella, Anthony J. ${ }^{1}$

${ }^{1}$ University of Ottawa, Division of Urology, Ottawa, ON, Canada; ${ }^{2}$ Urology Centers of Alabama, Birmingham, AL, United States

Introduction and Objectives: Mulhall et al have reported the incidence of climacturia (involuntary loss of urine during sexual activity) after radical prostatectomy approaches 20 percent of men. Cancer survivorship quality of life is negatively affected by climacturia, and the introduction of the male sling for incontinence may provide resolution of these symptoms, based on recent reports. Unfortunately, a significant proportion of urologists remain unaware of climacturia, thereby limiting potential benefit. We report on senior resident awareness of climacturia, institutional practices, and potential treatments.

Methods: Twenty-nine residents completed the 2010 prosthetics course and multipart climacturia questionnaire. These trainees represent a broad United States geographic distribution. The climacturia component consisted of 10 multipart questions (the majority Likert-scaled 1-10), addressing residency experience at respective institutions.

Results: The majority of residents were in the 4th year of residency. Climacturia was discussed with patients, prior to surgical treatment for localized $\mathrm{CaP}$, in 5/29 cases. 14/29 respondents were aware of climacturia prior to the course, but pre-/post RP discussions with the patient did not address the involuntary loss of urine. Inconsistencies in rates were reported by those aware, ranging from 0 to 80 percent (cluster around $10 \%$ ), as well as potential impact on patient QoL (Likert range 1-10, $80 \%$ report 7-10), time to resolution (range out to 36 months) and treatments available (Kegel, AUS, male sling, condom, Actis band, bulking agents). Conclusions: Post-radical prostatectomy quality of life is meaningfully impacted by involuntary loss of urine during sexual activity. Contemporary residency programs do not consistently address climacturia as part of the postoperative side-effects secondary to RP; there is a need for awareness and education programs focused on trainees, as well as patients undergoing RP.

\section{UP-092}

Post-prostatectomy Penile Rehabilitation and Erectile Dysfunction Treatment: Senior Resident Perceptions and Institutional Practices Based on Results Obtained at a Resident Training Laboratory Zappavigna, Christopher; Bella, Anthony J.

University of Ottawa, Division of Urology, Ottawa, ON, Canada

Introduction and Objectives: Mulhall et al recently published findings of US urological practice for post-radical prostatectomy recovery of erectile function (penile rehabilitation, PR). The Sexual Medicine Society of North America (SMSNA) provides a hands-on prosthetics training course as part of the annual fall meeting for senior residents; we present contemporary data for PR and treatment of post-prostatectomy erectile dysfunction (ED) in this cohort. Methods: Twenty-nine residents completed the 2010 prosthetics course and multipart post-RP ED questionnaire, representing broad US geographic distribution. The penile post RP/ED component consisted of 20 questions (majority Likert-scaled 1-10), addressing different residency experiences.
Results: The majority of residents were in the 4th year of residency. ED was discussed with patients, prior to treatment for localized CaP, in all cases although this varied from a short, broad concept discussion to detailed post-RP choices and early initiation of treatment. Postoperative potency rates given to the patient before surgery were $50-100,30-75$, and $0-50$ for bilateral, unilateral, and non-nerve sparing procedures, respectively. PDE5, intracorporeal injection, MUSE and vacuum erection device use varied, as did timing of initiation, and frequency of use. In most cases, a rehabilitation strategy (27/29 PDE5 based) is initiated within 1 week of catheter removal. Intra-institutional variabilities noted as well, which were surgeon or program dependent.

Conclusions: Post-radical prostatectomy PR remains controversial, as the optimal type of intervention, timing, duration, and functional outcomes vary across studies or are unknown. Concern is raised regarding the expectations for recovery, as communicated to the patient, which in many cases are quite higher than reported. Prospective practice-specific outcomes would allow for more accurate patient counseling and more realistic recovery expectations.

\section{UP-093}

Leadership in Canadian Urology: What Is the Right Stuff?

Robinson, Michael; MacNeily, Andrew; McInnes, Colin; Lennox, Peter; Carr, Nicholas; Skarlicki, Daniel; Masterson, John; Arneja, Jugpal University of British Columbia, Vancouver, BC, Canada

Introduction and Objectives: There is little data characterizing leadership roles within Canadian Urology. The importance of these positions in Urology underscores the need for further investigation in order to provide insight for recruitment, development and success.

Methods: All Canadian Urology program directors and division/department heads were invited to complete an online leadership survey as part of a larger national cohort from 11 other surgical specialties.

Results: Response rate was $62 \%(13 / 21)$, the majority of whom were Caucasian $(77 \%)$ and male $(92 \%)$. Only $8 \%$ of respondents in Urology hold an advanced degree compared to $45 \%$ in other specialties. Additional leadership training was done by $54 \%$. Residency was completed in Canada by $92 \%$, but $62 \%$ completed fellowships abroad. A majority reported no well-defined job description for their role (54\%). The top responsibility reported by leaders was mentoring residents $(67 \%)$, followed by advising staff (62\%). Excellence in patient care and teaching were seen as the most important professional characteristics, while integrity was the personal quality felt most important. Leaders reported $17 \%$ of their income came from their leadership role, equivalent to the time required for position duties (19\%). "Time management" was listed as the greatest challenge faced (54\%). Leadership style was reported as "democratic" by $92 \%$. Leaders in urology most often self-rate their leadership skills lower than leaders from other surgical specialties (4 vs. $3 / 10)$. Conclusions: Positions of leadership in Urology are disproportionately represented by Caucasian males and comparatively few hold relevant advanced degrees. Excellence in the areas of teaching and patient care, and high personal integrity are felt to be the most important characteristics for success. Time management issues are viewed as the greatest challenge. This preliminary data should prove useful for the mentoring, recruitment and success of future leaders in our specialty.

\section{UP-094}

What Is the Best Local Anesthesia during Extracorporeal Shockwave Lithotripsy? Prospective Randomized Clinical Study Comparing EMLA and Lidocaine

Mallat, Faouzi; Hmida, Wissem; Hidoussi, Adnen; Slama, Adel; Jaidane, Mehdi; Ben Sorba, Nebil; Mosbah, Ali Faouzi

Department of Urology, Sahloul, Sousse, Tunisia

Introduction and Objectives: Determine the best method of local anesthesia (EMLA versus Lidocaine) during extracorporeal shockwave lithotripsy (ESLW) procedure.

Methods: A prospective, randomized, single blind and crossover study was performed in 96 patients who were scheduled for ESWL for kidney or ureter stones over a period of six months (January-June 2010). Patients were divided into 3 groups, group 1 including 32 patients who received 
EMLA ( $5 \%$ ) at a dose $5 \mathrm{~g}, 15$ minutes before the ESWL, group 2 including 32 patients who received $20 \mathrm{ml}$ of Lidocaine $(1 \%)$ injected subcutaneously at the target area and group 3, including 32 patients, who received placebo as a neutral gel applied in the same way as EMLA. Pain was measured by clinical parameters (blood pressure, heart rate, and vagal signs) and with 3 pain scales (visual analogical scale score (VAS), Verbal rating scale (VRS) and numeric rating scale (NRS)). Statistical analysis was performed with Chi 2, Student and Annova tests.

Results: The average age was 49 years. During the session of ESWL, there was an increase in systolic blood pressure and heart rate in the 3 study groups. The difference was statistically significant only for the vagal signs in the crossover studies EMLA / placebo and Lidocaine/EMLA. The analytical study confirms that the variation of the average value of the three pain scales during different times of the ESWL was statistically significant in the 3 groups. Four predictors of pain were found: the size of the stone $(p=0.021)$, frequency $(p=00.36)$ and wave intensity $(0.036)$ shock and the duration of the session (0.036). The level of pain, in the three scales was less important for the Lidocaine group compared to placebo and the EMLA groups.

Conclusions: The ESWL is a painful procedure; the pain may influence the effectiveness of the act. The injection of Lidocaine during ESWL was the best method of local anesthesia compared to the EMLA cream and placebo.

\section{UP-095}

\section{Trans-splenic Percutaneous Nephrolithotomy with Secondary Procedure via the Same Tract}

Bhojani, Naeem; Mandeville, Jessica A.; Boris, Ronald S.; Lingeman, James $\mathrm{E}$.

Indiana School of Medicine, Department of Urology, Indianapolis, IN, United States

Introduction and Objectives: Splenic injury is a known complication of upper pole (UP) percutaneous nephrolithotomy (PNL). We describe a case of trans-splenic PNL followed by secondary PNL via the same tract. Methods: A 62-year-old man presented with hematuria and bothersome lower urinary tract symptoms despite daily tamsulosin. Rectal exam was normal. A computed tomography (CT) scan revealed left hydronephrosis with a large stone burden (Fig. 1). The patient underwent holmium laser enucleation of the prostate (HoLEP) and left PNL.

Results: HoLEP was performed first. Operative time was $25 \mathrm{~min}$ and blood loss (EBL) was $5 \mathrm{cc}$. A $5 \mathrm{Fr}$ catheter was advanced into the left kidney and he was positioned prone. Access to the UP was obtained with an 18 gauge needle. The tract was balloon dilated and a $30 \mathrm{Fr}$ access sheath was seated Stone removal was performed with rigid and flexible instruments. A $10 \mathrm{Fr}$ looped nephrostomy tube (NT) was placed. Fluoroscopic imaging did not reveal evidence of pleural effusion. PNL operative time was $227 \mathrm{~min}$ and EBL was 50cc. Non-contrast CT on postoperative day (POD) \#1 revealed the percutaneous tract traversed the spleen (Fig. 2). No hematomas were identified. Several $2-3 \mathrm{~mm}$ stones remained. POD \#1 Hb was $14.8 \mathrm{~g} / \mathrm{dL}$ (preoperative 15.3). He remained stable and was taken for a secondary $\mathrm{PNL}$ on POD\#2. The tract was re-dilated with a $26 \mathrm{Fr}$ rigid dilator and a $26 \mathrm{Fr}$ sheath was seated in the UP. All stones were extracted and a $10 \mathrm{Fr}$ NT was replaced. Operative time was $53 \mathrm{~min}$ and EBL was 10cc. POD\#2 $\mathrm{Hb}$ was $14.9 \mathrm{~g} / \mathrm{dL}$. He was discharged on POD $\# 3$ with the NT in place. The NT was removed on POD $\# 14$.

Conclusions: In stable patients, post-PNL splenic injury can be managed conservatively. In select patients, secondary PNL via the tract may be feasible.

\section{UP-096}

Uric Acid Stone Prevalence Doubles in the Severely and Morbidly Obese (BMI>35) Compared to the Overweight (BMI 25-30)

Sivalingam, Sri; Penniston, Kris; Nakada, Stephen Y.

University of Wisconsin, Madison, WI, United States

Introduction and Objectives: We sought to identify correlations between body mass index (BMI) and stone type, with particular emphasis on severe obesity (BMI $>35)$ and morbid obesity (BMI $>40)$.
Methods: Retrospective review of our institutional stone composition database was performed between March 2006 and Sept 2010 with IRB approval, and patients with BMI data were included. Patients were grouped by BMI: $<25$ (normal), 25-30 (overweight), 30-35 (obese), 35-40 (severely obese) \& >40 (morbidly obese). Stone type was defined by the predominant stone component in each analysis (COM and COD were grouped as calcium oxalate; and brushite stones were grouped as calcium phosphate).

Results: Two hundred patients were included in the analysis. The distribution of patients and mean age (MA) were as follows: normal BMI $(n=58,29 \% ; M A=43)$, overweight $(n=35,17.5 \% ; M A=54.9)$, obese $(n=47$, $23.5 \% ; M A=52.9)$, severely obese $(n=38,19 \% ; M A=52.6)$ and morbidly obese ( $n=22,11 \% ; M A=48)$. The prevalence of UA stones increased in each successive BMI group; notably, the rates in the severely and morbidly obese groups were significantly higher than that in the normal group ( $p=0.03$ ) and doubled in prevalence compared to the overweight. Conclusions: The prevalence of UA stones was highest in the severely and morbidly obese. While obesity portends an increased risk for UA stones, our prevalence data indicates extreme obesity as problematic.

\section{UP-097}

Targeted Intervention versus Conservative Intervention for the Prevention of Kidney Stone Recurrence

Arsovska, Olga; Wu, Christopher; Sutton, Roger A. L.; Paterson, Ryan F.: Chew, Ben

University of British Columbia, Vancouver, BC, Canada

Introduction and Objectives: Conservative advice for stone prevention consists of increasing fluid intake, limiting protein, sodium and oxalate, and moderating calcium intake. Alternatively, performing two-24 hour urine tests can highlight specific metabolic abnormalities and targeted approaches such as selective dietary restriction, potassium citrate, or thiazide diuretics can be tailored accordingly. Conservative therapy is less costly, however it is not known if it is as effective as targeted therapy in preventing kidney stones, which we sought to determine with this study. Methods: A retrospective chart review of patients from the Stone Centre at Vancouver General Hospital was performed of 98 patients with recurrent metabolic stone disease, consisting of 24-hour urine collections while on a random diet and again after dietary modification. Sex, age, body mass index (BMI), medical risk factors, anatomical risk factors, followup length, stone recurrences, and changes in volume, calcium, oxalate, citrate, sodium, and uric acid in the 24-hour urine samples were compared between targeted and conservative treatment groups.

Results: There was no difference observed in urinary oxalate, calcium, or citrate between groups. Urinary sodium $(p=0.014)$ and uric acid $(p<0.001)$ increased for the targeted metabolic management. In the obese population, there was a significant increase in the urinary sodium $(p=0.003)$ and volume $(p=0.034)$ for the targeted treatment group. There was no difference in stone recurrence rates between groups with a mean follow-up of 6.8 months $(p=0.391)$.

Conclusions: From our results, both approaches in the management of patients at our centre did not affect stone recurrence rates. Urinary calcium levels did not increase despite increases in urinary sodium. Longer followup will determine which approach is more useful in stone prevention.

\section{UP-098}

Pulsed Fluoroscopy in Ureteroscopy and Percutaneous Nephrolithotomy

Elkoushy, Mohamed; Andonian, Sero

McGill University Health Centre, Montreal, QC, Canada

Purpose: To assess the impact of pulsed fluoroscopy on the total fluoroscopy time during ureteroscopy (URS) and percutaneous nephrolithotomy (PCNL).

Introduction and Objectives: A retrospective review of prospectively collected data was performed for consecutive patients undergoing URS and PCNL by a single surgeon between July 2009 and July 2011. Pulsed Fluoroscopy (PF) at a rate of 4 frames per second ( $4 \mathrm{fps}$ ) was routinely used in all URS procedures since January 2011 and in all PCNL pro- 
Table 1. UP-098. Patient, stone and operative characteristics in URS patients

\begin{tabular}{|c|c|c|c|}
\hline Variable & $\begin{array}{l}\text { Standard fluoroscopy (30 fps) } \\
\qquad(\mathrm{n}=117)\end{array}$ & $\begin{array}{c}\text { Pulsed } \\
\text { fluoroscopy } \\
(4 \mathrm{fps})(\mathrm{n}=46)\end{array}$ & $p$ value \\
\hline Gender (male) & $78(66.7 \%)$ & $31(67.4 \%)$ & 0.93 \\
\hline Age (years) mean $(95 \% \mathrm{Cl})$ & $53.2(50.5-55.9)$ & $54.2(49.8-58.6)$ & 0.70 \\
\hline BMI (kg/m2) mean $(95 \% \mathrm{Cl})$ & $27.28(18.95-39.65)$ & $26.42(21.95-33.84)$ & 0.20 \\
\hline Laterality (Left) & $61(52.1 \%)$ & $28(60.9 \%)$ & 0.32 \\
\hline Stone size $(\mathrm{mm})$ mean $(95 \% \mathrm{Cl})$ & $12.9(11.1-14.7)$ & $10.2(8.7-11.6)$ & 0.03 \\
\hline \multirow{4}{*}{$\begin{array}{l}\text { Ureteral } \\
\text { Renal } \\
\text { Both }\end{array}$} & $56(47.9 \%)$ & $23(50.0 \%)$ & \multirow{3}{*}{0.74} \\
\hline & $43(36.7 \%)$ & $13(28.3 \%)$ & \\
\hline & $18(15.4 \%)$ & $10(21.7 \%)$ & \\
\hline & $40(34.2 \%)$ & $15(32.6 \%)$ & 0.85 \\
\hline Full/ partial staghorns & $4(3.4 \%)$ & $1(2.2 \%)$ & 1.00 \\
\hline Radiolucent stones & $15(12.8 \%)$ & $6(13.0 \%)$ & 1.00 \\
\hline Preoperative stents & $70(59.8 \%)$ & $28(60.9 \%)$ & 0.90 \\
\hline Balloon dilations & $9(7.7 \%)$ & $5(10.9 \%)$ & 0.54 \\
\hline Access sheath & $61(55 \%)$ & $18(40 \%)$ & 0.11 \\
\hline \multirow{3}{*}{$\begin{array}{ll} & \text { Flexible } \\
\text { URS type } & \text { Semi-rigid } \\
& \text { Both }\end{array}$} & $44(39.6 \%)$ & $10(21.7 \%)$ & \multirow{3}{*}{0.06} \\
\hline & $29(26.1 \%)$ & $10(21.7 \%)$ & \\
\hline & $38(34.2)$ & $26(56.5 \%)$ & \\
\hline Mean OR/min $(95 \% \mathrm{Cl})$ & $76.9(70.8-83.0)$ & $77.0(66.9-87.0)$ & 0.95 \\
\hline Residual stone & $22(18.8 \%)$ & $9(19.6 \%)$ & 0.91 \\
\hline Mean fluoroscopy time $/ \mathrm{sec}(95 \% \mathrm{Cl})$ & $109.1(94.2-123.9)$ & $44.1(36.5-51.6)$ & $<0.001$ \\
\hline
\end{tabular}

cedures since November 2010. Patients were divided into 2 groups for each procedure based on whether SF or PF was used. Patient and stone characteristics together with operative data were compared between both groups of each procedure using univariate and multivariate analyses to correct for patients, stone and surgical variables.

Results: A total of 163 URS (117 SF and 46 PF) and 100 PCNL (50 SF and $50 \mathrm{PF}$ ) were included. In the URS cohort, there were no significant differences between both SF and PF groups in terms of age, gender, $\mathrm{BMI}$, stone location, and multiplicity $(\leq 0.20)$. The SF group in the URS cohort had significantly larger stone size (12.9 vs. $10.2 \mathrm{~mm} ; p=0.03)$, which lost its significance in the multivariate analysis. Duration of surgery and stone-free rates were also comparable in both groups of URS and PCNL ( $\leq 0.06)$. Compared to PF groups, patients in the SF groups were exposed to a mean of 65 and 220 seconds more fluoroscopy (109.1 vs. $44.1 \mathrm{sec}, p<0.001)$ and $(341.1 \mathrm{vs} .121 .5 \mathrm{sec}, p<0.001)$ in the URS and PCNL cohorts, respectively. These differences in mean fluoroscopy time retained their significance in the multivariate analyses $(p<0.001)$. In the multivariate model, female gender, right-sided stones, increased number of punctures and postoperative stenting were significantly higher in the PF in PCNL group (Table 1, Table 2).

Conclusions: The use of pulsed fluoroscopy during URS and PCNL was associated with significantly lower fluoroscopy time thus, reducing radiation exposure.

\section{UP-099}

Laparoscopic Nephrectomy with Intact Specimen Extraction for Massive Polycystic Kidney Disease: Updated Technique and Outcome Analysis

Bansal, Rahul Kumar; Tu, Hin Yu Vincent; Kapoor, Anil

McMaster Institute of Urology, St. Joseph's Healthcare Hamilton, Hamilton, ON, Canada

Introduction and Objectives: We present our technique of laparoscopic nephrectomy with intact specimen extraction for patients with massively enlarged autosomal-dominant polycystic kidney disease (ADPKD).
Methods: We reviewed all laparoscopic nephrectomies with intact specimen extraction done for ADPKD, in a university hospital between April 2004 and January 2011. We used three $10 \mathrm{~mm}$ ports with additional one or two $5 \mathrm{~mm}$ ports in our technique of transperitoneal laparoscopic nephrectomy.

Results: Total of $39(\mathrm{~L}=14, \mathrm{R}=25)$ laparoscopic nephrectomies were performed in 32 patients $(M=21, F=11)$ with ADPKD. The indications were to create space for future renal transplant in $21(54 \%)$, pain in $16(41 \%)$, recurrent urosepsis in $2(5 \%)$, bleeding requiring transfusions in $2(5 \%)$ and renal tumor in one $(2.5 \%)$ renal unit. Mean age and body mass index were 52.2 years (range $29-72$ years) and $26.9 \mathrm{~kg} / \mathrm{m} 2$ (range 21.6-34.0 $\mathrm{kg} / \mathrm{m} 2)$ respectively. Fourteen $(36 \%)$ patients had previous abdominal surgeries and $26(81 \%)$ patients were on dialysis. No patient had preoperative angioembolization of the renal unit. Mean preoperative hemoglobin and serum creatinine levels were 131.6g/L (range 107-171g/L) and $514 \mu \mathrm{mol} / \mathrm{L}$ (range $84-923 \mu \mathrm{mol} / \mathrm{L}$ ) respectively. Mean operative time and estimated blood loss were 185 minutes (range 113-287 minutes) and $94 \mathrm{~mL}$ (range $10-350 \mathrm{~mL}$ ) respectively. No patient required open conversion. Mean specimen size was $24.2 \mathrm{~cm}$ (range $15-38 \mathrm{~cm}$ ), weight $1515 \mathrm{~g}$ (range 412-4590g) and length of extraction incision was $9.2 \mathrm{~cm}$ (range $6-13 \mathrm{~cm})$. There was one $(2.5 \%)$ intraoperative and three $(7.5 \%)$ postoperative complications. There were no deaths. Mean length of hospital stay was 5 days (range $3-12$ days).

Conclusions: Our technique of transperitoneal laparoscopic nephrectomy for patients with ADPKD is safe and offers all the advantages of minimally invasive surgery such as reduced blood loss, shorter incision, excellent cosmesis and faster recovery. 


\section{Table 2. UP-098. Patient, stone and operative characteristics in PCNL patients}

\begin{tabular}{|c|c|c|c|c|}
\hline \multicolumn{2}{|c|}{ Variable } & $\begin{array}{c}\text { Standard fluoroscopy } \\
\text { (30 fps) }(n=50)\end{array}$ & $\begin{array}{l}\text { Pulsed fluoroscopy } \\
(4 \mathrm{fps})(\mathrm{n}=50)\end{array}$ & $p$ value \\
\hline \multicolumn{2}{|l|}{ Gender (male) } & $36(72 \%)$ & $23(46 \%)$ & 0.01 \\
\hline \multicolumn{2}{|c|}{ Age (years) mean $(95 \% \mathrm{Cl})$} & $53.7(49.4-59.6)$ & $55.7(49.6-59.6)$ & 0.53 \\
\hline \multicolumn{2}{|c|}{ Laterality (Left) } & $39(78 \%)$ & $20(40 \%)$ & $<0.001$ \\
\hline \multicolumn{2}{|c|}{ Stone size $(\mathrm{mm})$ mean $(95 \% \mathrm{Cl})$} & $31.8(26.7-42.5)$ & $33.2(29.8-39.4)$ & 0.70 \\
\hline Stone location & Both & $11(22 \%)$ & $15(30 \%)$ & 0.67 \\
\hline \multirow{3}{*}{$\begin{array}{l}\text { Multiplicity } \\
\text { of stones }\end{array}$} & Single & $16(32 \%)$ & $8(16 \%)$ & \multirow{3}{*}{0.29} \\
\hline & Multiple & $18(36 \%)$ & $26(52 \%)$ & \\
\hline & Stag/partial & $16(32 \%)$ & $16(32 \%)$ & \\
\hline PCNL position & Supine & $3(6 \%)$ & $4(8 \%)$ & 1.00 \\
\hline \multirow{4}{*}{$\begin{array}{l}\text { Number of } \\
\text { punctures }\end{array}$} & None & $11(22 \%)$ & $6(12 \%)$ & \multirow[t]{3}{*}{0.02} \\
\hline & One puncture & $22(44 \%)$ & $10(20 \%)$ & \\
\hline & $\geq 2$ punctures & $17(34 \%)$ & $34(68 \%)$ & \\
\hline & Used previous & $10(20 \%)$ & $6(12 \%)$ & \multirow[t]{3}{*}{0.08} \\
\hline \multirow[t]{2}{*}{ Number of tracts } & One tract & $35(70 \%)$ & $32(64 \%)$ & \\
\hline & 2 tracts & $5(10 \%)$ & $12(24 \%)$ & \\
\hline \multicolumn{5}{|l|}{ Tract location* } \\
\hline & Upper pole & $5(12.5 \%)$ & $17(38.6 \%)$ & \multirow[b]{2}{*}{0.018} \\
\hline & Mid pole & $15(37.5 \%)$ & $14(31.8 \%)$ & \\
\hline
\end{tabular}

*The pre-formed tracts in both groups were excluded from the analysis. URS: ureteroscopy; BMI: body mass index; Cl: confidence interval; PCNL: percutaneous nephrolithotomy.

\section{UP-100}

Urologists in Cyberspace: Evaluating Quality of American Urologists' Websites on the Internet

Yan, Hanmu; Margel, David; Fleshner, Neil; Wong, Lih-Ming

Department of Urology, Princess Margaret Hospital, Toronto, ON, Canada Introduction and Objectives: With increased access to the Internet, society is increasingly seeking health information online. Urologists use websites to facilitate information dissemination but also to promote their own services. In this study, we use a validated tool, the Health on the Net Foundation code of conduct (HONcode), to evaluate a selection of websites from urologists' based in the United States of America (USA). Methods: The 10 most populous cities in America were identified from the US Census Bureau. Using the Internet search engine, www.google. com, we searched "urologist + city" and identified the top ten hits for each city. Each website was scored using the HONcode (15 points). The median score was used to dichotomize the cohort and multivariable logistic regression used to identify independent predictors of higher scores.

Results: Of the total 78 websites analyzed, there were 18 academic institutions, 43 group and 17 solo practices. A medical website design service had been used by 18 websites. The HONcode badge was seen on 3 websites $(4 \%)$. The median HON code score was 5.5 (range 1-10). Multivariable logistic regression showed academic centre was a predictor of high HON score (OR 7.7, $\mathrm{Cl} 1.5-39.8, p=0.014)$ and use of medical website design service associated with lower HON score (OR $0.08, \mathrm{Cl}$ $0.18-0.33, p=0.001$ ).

Conclusions: Using a validated tool for appraising online health information, we found a wide variation in quality of urologists' websites in America. Better awareness of available services and standards, and guidance from governing bodies would improve quality of websites and the overall credeibility of urology as a specialty.

\section{UP-101}

Robotic-assisted Microsurgery: the Initial 603 Case Experience Gudeloglu, Ahmet; Priola, Karen; Parekattil, Sijo

University of Florida and Winter Haven Hospital, Winter Haven, FL, United States

Introduction and Objectives: Since the introduction of the operating microscope in 1975, microsurgeons continue their quest for better outcomes and improved operative ease. Use of the daVinci Si system (Intuitive Surgical Inc., Sunnyvale, CA) for microsurgery may provide advantages in terms of offering magnification, motion scaling and additional surgical arms in a stable ergonomic platform. This study presents our 603 case experience of robotic assisted microsurgery by a single fellowship-trained microsurgeon (Jul'07 - Jan'12). 
Methods: Cases included: 65 robotic vasovasostomy (RAVV), 42 robotic vasoepididymostomy (RAVE), 354 robotic microsurgical denervation of the spermatic cord (RMDSC) for chronic orchialgia, 2 robotic testicular artery micro-anastomosis (injured during RMDSC), 128 robotic subinguinal varicocelectomies, 10 robotic micro-dissection testicular sperm extractions and 2 robotic microsurgical nerve grafting during robotic prostatectomy. The 4-arm Si robotic system was utilized with high definition magnification (10-15x), micro robotic instruments, and a new micro robotic Doppler probe.

Results: All cases were completed successfully without a trained microsurgical assistant. The fourth arm improved surgeon efficiency (extra microsurgical instrument handled simultaneously). Previously, the microsurgeon could perform only two standard microsurgical procedures a day due to fatigue limitations using the standard microscope. With the aid of dual robotic systems, the same microsurgeon has been able to routinely perform up to 10 microsurgical procedures a day due to the ergonomic advantages of the robot.

Conclusions: Robotic assisted microsurgery is in its infancy. However with technical advances, a number of microsurgical procedures can be successfully performed with improved efficiency.

\section{UP-102}

Assessment of the Understanding of Androgen Deprivation Therapy (ADT) Associated Side Effects in Primary Care Physicians Soeyonggo, Tony ${ }^{1}$; Locke, Jennifer ${ }^{1}$; Del Giudice, Lisa ${ }^{2}$; Alibhai, Shabbir ${ }^{3}$; Fleshner, Neil ${ }^{4}$; Warde, Padraig

${ }^{1}$ Faculty of Medicine, University of Toronto, Toronto, ON, Canada; ${ }^{2}$ Department of Family and Community Medicine, Sunnybrook Health Sciences Centre, Toronto, ON, Canada; ${ }^{3}$ Department of Medicine, University Health Network, Toronto, ON, Canada; ${ }^{4}$ Department of Surgery (Urology), Princess Margaret Hospital, Toronto, ON, Canada; ${ }^{5}$ Department of Radiation Oncology, Princess Margaret Hospital, Toronto, ON, Canada Introduction and Objectives: Androgen Deprivation Therapy (ADT) is a common treatment for men with prostate cancer, but has a number of toxicities. In this study, we assessed the knowledge of primary care physicians (PCPs) regarding ADT associated side effects and their interest in increasing knowledge in this area.

Methods: We obtained a list of active PCPs across Canada using the Canadian Medical Directory and distributed a cross-sectional survey to 600 randomly selected physicians. The survey collected basic demographic information, whether they had patients on ADT in their practice, their knowledge level of ADT side effects, and their interest in educational resources/opportunities to improve their knowledge base.

Results: Seventy-one completed questionnaires were returned; 52 respondents provided family medicine care to adults. Sixty-five percent of respondents were male, with a median age between $51-60$ years old (range $<30$ to $>60$ years old) and median number of years in practice between $21-30$ years (range $<10$ to $>30$ years). Most physicians $(86.5 \%)$ had patients on ADT for prostate cancer in their practice. Forty percent felt their knowledge of ADT side effects was inadequate and $50 \%$ felt uncomfortable counseling patients on this topic. The majority $(74.5 \%)$ reported that they rarely or never discuss ADT toxicities with patients. Eighty-five percent of PCPs wanted additional educational resources on ADT. With regards to avenues of information on ADT, physicians in our study expressed an interest in continued medical education (CME) events $(46.2 \%)$, educational pamphlets $(32.7 \%)$ and specialist consultations $(26.9 \%)$.

Conclusions: While ADT use is commonly seen in primary care practice, many PCPs feel that they have limited knowledge on how to counsel patients with ADT-associated side effects. There is a strong interest to learn about ADT side effects through CME events and educational pamphlets.

\section{UP-103}

Senior Resident Perceptions of Industry Interaction with Prosthetic Urologists Based on Results Obtained at a Resident Training Laboratory

Zappavigna, Christopher; Bella, Anthony J.

University of Ottawa, Division of Urology, Ottawa, ON, Canada

Introduction and Objectives: There is little in the urologic literature that provides information on the interactions between trainees and company representatives from the urologic prosthetics industry.

Methods: Twenty-nine residents completed the 1010 prosthetics course and multipart industry interaction questionnaire. These trainees represent a broad United State geographic distribution.

Results: The residents were asked questions focused on several aspects of industry-resident interaction as it pertains to GU prosthetics. Specific areas of interest were access to the resident at their training institution $(>80 \%)$, whether this was limited to OR exposure (approximately half), the utility of these industry contacts (bias towards company products vs. educational prosthetic messaging) as it relates to male slings, AUS and penile prostheses (19/29 scored the maximum 10$)$, as well as perception of ethics and potential for bias. The majority of respondents are of the opinion that these interactions can be done in an ethical manner. Access post-training for company representatives to office (23/29) and OR (25/29) were also addressed.

Conclusions: There have been several recent publications that have called to question the utility of industry-surgeon interaction, and the potential for negative impact on patient care. These results suggest that residents perceive benefit through these relationships with minimal ethical concerns, if care is taken to minimize promotional aspects and focus on improving health care delivery to patients through surgeon and patient education in its many forms.

\section{UP-104}

Factors Affecting Surgical Performance and Operating Room Safety in Urology: a Preliminary Evaluation

Lee, Jason Y.'; Gettman, Matthew ${ }^{2}$; Landman, Jaime ${ }^{3}$; McDougall, Elspeth M. ${ }^{3}$; Sundaram, Chandru P. ${ }^{4}$; Sweet, Robert ${ }^{5}$; Zorn, Kevin ${ }^{6}$

${ }^{1}$ St. Michael's Hospital, University of Toronto, Toronto, ON, Canada; ${ }^{2}$ Mayo Clinic, Rochester, MN, United States; ${ }^{3}$ University of California Irvine, Orange, CA, United States; ${ }^{4}$ Indiana University, Indianapolis, IN, United States; ${ }^{5}$ University of Minnesota, Minneapolis, MN, United States; ${ }^{6}$ University of Montreal (CHUM), Montreal, QC, Canada

Introduction and Objectives: Surgical outcomes depend not only on patient \& disease factors but the skill of the surgical team. A survey was conducted to evaluate various factors in the OR environment that impact an individual surgeon's "performance".

Methods: An internet-based survey was distributed to 2057 urologists regarding factors that potentially impact surgical outcomes by serving as surgical distractors; surgeon-specific (internal), environmental (external), and interprofessional (interactive).

Results: 324 urologists $(16 \%)$ completed the survey; $70 \%$ were N. American, 43\% were from academic institutions and $68 \%$ had completed a clinical fellowship. The majority of urologists reported having operated when sleep deprived (80\%), when significantly ill (86\%), with a MSK injury $(57 \%)$, or under significant social stress $(67 \%)$. While up to $37 \%$ reported such internal distractors had significantly affected technical performance (e.g. slower OR times), only $23 \%, 45 \%, 42 \%$, and $32 \%$ of these urologists had ever cancelled an OR day due to fatigue, illness, MSK injury or social stressor, respectively. When in the OR, music was played routinely by $56 \%$ of urologists and $70 \%$ reported answering pages and discussing consults. 25\% reported working "commonly" with scrub nurses that were unfamiliar with the procedure or instruments, only $43 \%$ had a consistent OR assistant, and $64 \%$ reported that the scrub nurse would "commonly" or "sometimes" scrub out during a critical portion of the surgery. Overall, $82 \%$ felt the OR environment required changes to improve safety and $13 \%$ reported that a complication had occurred mainly due to such external distractors. Urologists working at hospitals where a routine preoperative "time-out" was performed reported fewer complications relating to external distractors. 
Conclusions: Various factors may significantly affect technical performance and the OR environment in urology, promoting undesirable patient outcomes. Such factors must be considered in order to optimize patient care

\section{UP-105}

\section{The Management of Post-transplant Lymphoceles: Changes in} Practice at I'Hôtel-Dieu de Québec

Deschênes-Rompré, Marie-Pier; Diallo, Yoro; DeSerres, Sacha; Houde, Isabelle; Caumartin, Yves

Centre Hospitalier universitaire de Québec, Quebec, QC, Canada Introduction and Objectives: Lymphocele is a common complication following kidney transplantation. In our experience, lymphocele management has dramatically evolved overtime toward a less invasive approach The purpose of this study is to review efficacy and complications associated with their treatment.

Methods: Between January 1990 and December 2010, 962 kidney transplantations have been performed in our institution. 152 recipients $(15.8 \%)$ developed a lymphocele postoperatively. Characteristics of recipients and donors, peritransplant details, long-term function and survivals were retrospectively collected. Descriptive analysis of patients who developed a posttransplant lymphocele has been performed to assess efficacy/complications of different management options.

Results: From the 152 patients presenting a post-transplant lymphocele, $78 \%$ required an active treatment. Aspiration was the most frequently used (52\%) but was associated with a low success rate $(15 \%)$. Indwelling drainage improved the success rates up to $34 \%$ but was associated with higher risk for infectious complications (14\%). At first, internal marsupialisation of the lymphocele was the operation of choice for refractory lymphoceles and was necessary for $44 \%$ of our symptomatic patients with a $50 \%$ success rate and a $17 \%$ complication incidence. Later, sclerotherapy with proviodine was used in $35 \%$ of our cohort with a high success rate of $97 \%$ and a complication rate of $5 \%$.

Conclusions: In our experience, posttransplant lymphocele management has evolved toward a minimally invasive approach with proviodine sclerotherapy. This treatment strategy has been associated with a high success rate and low incidence of complications. Consequently, we think this strategy should be favored in the treatment of symptomatic lymphoceles.

\section{UP-106}

Factors Associated with an Increased Risk of Delayed Graft Function in Donation after Cardiac Death Kidneys

Boyle, Shawna'; McGregor, Thomas B. ${ }^{2}$; Moser, Mike ${ }^{3}$; Sharpe, Mike'; Luke, Patrick

${ }^{1}$ The University of Western Ontario, Department of Surgery, Division of Urology, London, ON, Canada; ${ }^{2}$ University of Manitoba, Department of Surgery, Division of Urology, Transplant and MIS, Winnipeg, MB, Canada ${ }^{3}$ The University of Western Ontario, Department of Surgery, Division of Urology and Transplant, London, ON, Canada

Introduction and Objectives: The purpose of this study was to review the experience of our donation after cardiac death (DCD) kidney program to determine factors that may be associated with an increased risk of delayed graft function (DGF).

Methods: We retrospectively analyzed the charts of all 63 patients receiving single-kidney-only transplant of a DCD kidney since our DCD programs inception in 2006. DGF was seen in 41 of 63 patients $(65 \%)$ and there were no cases of primary non-function.

Results: Indications for dialysis had some overlap and included fluid overload (18/41), hyperkalemia (10/41), and no indication recorded or simply uremia in $17 / 41$. Patients with DGF were noted to have received donor organs with significantly longer time with systolic blood pressure (SBP) $<55 \mathrm{mmHg}$ (28.2 $\mathrm{min}$ vs. $21.1 \mathrm{~min}, p=0.049$ ) and a trend toward a longer time to asystole (29.3 min vs. $16.6 \mathrm{~min}, p=0.063)$. Only 2 of 13 grafts $(15.4 \%)$ showing satisfactory early function had SBP $<55 \mathrm{mmHg}$ for more than 30 minutes, compared to 20 out of $50(40 \%)$ if the time was under 30 minutes, although the difference was not statistically significant. Machine cold perfusion $(n=36)$ of the donor organs did not significantly improve the rate of DGF, however, it was associated with improved creati- nine clearance on days $3(12.6 \mathrm{vs} .7 .8 \mathrm{~mL} / \mathrm{min}, p=0.036)$ and 7 (20.0 vs. $8.7 \mathrm{~mL} / \mathrm{min}, p=0.003$ ) and patients receiving DCD kidneys that had been machine perfused were discharged sooner ( 16 vs. 11.5 days, $p=0.006$ ). Conclusions: Our results are comparable to those seen at other institutions. DCD kidneys from donors having a SBP $<55$ for more than 30 minutes are very unlikely to show early graft function. Machine cold perfusion seems to improve kidney function in the early post-transplant period in these DCD kidneys but does not mitigate the risk of DGF.

\section{UP-107}

Debilitating Lower Urinary Tract Symptoms in the Post-renal Transplant Population Can Be Predicted Pre-transplantation Dion, Marie; Cristea, Octav; Langford, Sarah; Luke, Patrick; Sener, Alp University of Western Ontario, London, ON, Canada

Introduction and Objectives: Overactive bladder $(\mathrm{OAB})$ and benign prostatic hyperplasia $(\mathrm{BPH})$ are common entities in the aging population, which may be masked by the low urine output of end-stage renal disease (ESRD). Sequelae of these disease processes may pose an underlying risk to renal allografts from episodes of retention and infections. Our objective was to determine the frequency and severity of LUTS in renal TX recipients and to determine if validated questionnaires could predict which patients will develop severe LUTS after TX.

Methods: All adult renal-TX recipients at our institution from 2005 to 2010 were invited to participate in this study via mailed questionnaires. The Overactive Bladder Questionnaire $(\mathrm{OAB}-\mathrm{Q})$ and International Prostate Symptom Score sheet (IPSS) were completed based on three time points: pre-TX, and 6 and 12 months post-TX. Overall scores were tabulated based on the returned surveys.

Results: Of 465 patients who underwent renal TX, 105 participated in the study $(22.6 \%)$. LUTS were common pre-TX (15\% on OAB-Q) and post-TX ( $31 \%$ and $23 \%$ at 6 and 12 months respectively). Health-related quality of life (HRQL) scores pre-TX were predictive of moderate to severe symptoms post-TX with an odds ratio of $11.2(95 \% \mathrm{Cl} 2.7-45.9, p=0.0012)$ at 6 months and $9.2(95 \% \mathrm{Cl} 2.0-41.8, p=0.0085)$ at 12 months. In male patients the IPSS found $40.8 \%$ of men had moderate to severe BPH symptoms pre-TX. When their post-TX symptoms were examined these patients were 9.4 times as likely to suffer moderate to severe symptoms as compared to patients with low IPSS scores at 12 months $(95 \% \mathrm{CI}$ 1.7-51.9, $p=0.0086$ ).

Conclusions: The use of validated LUTS questionnaires prior to renal TX predict which patients will suffer significant LUTS post-TX. Identification of patients at risk for LUTS could allow for screening of inappropriate TX candidates, and treatment of urologic symptoms avoiding complications, which could compromise renal allografts.

\section{UP-108}

Thymoglobulin Differentially Increases CDR Naive T-cell Proliferation Following Renal Transplantation

Ullah, Shahnoor ${ }^{1}$; Telfer, Siobhan²; Mok, Amy ${ }^{3}$; Jevnikar, Anthony ${ }^{4}$; Luke, Patrick ${ }^{2}$; Sener, Alp

${ }^{1}$ Schulich School of Medicine, The University of Western Ontario, London, ON, Canada; ${ }^{2}$ Department of Surgery, The University of Western Ontario, London, ON, Canada; ${ }^{3}$ Department of Microbiology and Immunology, The University of Western Ontario, London, ON, Canada; ${ }^{4}$ Department of Medicine, The University of Western Ontario, London, ON, Canada; ${ }^{5}$ Department of Surgery, Department of Microbiology and Immunology, The University of Western Ontario, London, ON, Canada

Introduction and Objectives: The risk of acute T-cell mediated rejection remains an obstacle in kidney transplantation despite aggressive immunosuppressive therapy with T-cell depleting agents such as Thymoglobulin (ATG). The etiology behind this rejection is not well understood and may be rooted in a disturbance in the balance between the reconstituting naïve, memory and regulatory T-cell phenotypes. This study evaluated the effects of ATG on various CD4 and CD8 T-cell subsets at an early period following renal transplantation.

Methods: Kidney transplant recipients were allocated into receiving Simulect (Control, $n=9)$ or ATG $(n=12)$ based upon their immunological risk. Blood samples were obtained before and at various time intervals for 
1 month after transplantation and stained using cell surface and intracellular markers and analyzed using flow cytometry.

Results: Early time points demonstrated that ATG readily depletes CD4 and CD8 T-cell populations $(p<0.05)$. CD4 memory T-cells were depleted in the ATG group on day $2(p<0.05)$, with CD4 memory effector and central T-cells depleted by Day $7(p<0.05)$. Although no overall difference in the CD4 naïve T-cell population, the CD4 naïve effector T-cell population was depleted on day 7 and 14 in the ATG group $(p<0.05)$ while CD4 naïve central T-cells were depleted by day $2(p<0.05)$. CD8 memory and naive T-cells continued to be depleted on day $28(p<0.05)$. With respect to proliferation rates, ATG treated patients had increased turnover of memory T-cells on day $14(p<0.05)$ and particularly memory T-effector cells on day $2(p<0.05)$. CD4 naive and CD4 regulatory T-cell populations also had increased rates of proliferation on day 2 in the ATG group which were greater than seen in the CD4 memory population $(p<0.05)$.

Conclusions: This is the first study to provide novel information on the early proliferation of T-cells following ATG in kidney transplant recipients and may offer insight into future maintenance immunotherapy.

\section{UP-109}

Retubularization of the Ileocystoplasty Pouch for Conversion into an Ileal Conduit Diversion

Massaro, Peter; Gajewski, Jerzy; Bailly, Greg

Department of Urology, Dalhousie University, Halifax, NS, Canada

Introduction and Objectives: We present the outcomes and long-term follow-up of patients who failed initial ileocystoplasty and subsequently underwent conversion to an ileal conduit urinary diversion utilizing the retubularized pouch from the initial bladder augmentation.

Methods: The charts of all patients who underwent this surgery at our centre were reviewed. The indications for surgery, work-up, clinical outcomes, and complication rates were assessed. Patient reported symptom response based on global response assessment (GRA) was determined and used as a subjective method to assess overall treatment effectiveness. Results: Twelve patients with either interstitial cystitis/bladder pain syndrome (10) or neurogenic bladder (2) were followed for a mean of 71 months. The most common indication for surgical conversion was ongoing severe lower urinary tract symptoms or bladder pain despite several prior therapies. Operative and early complications were uncommon; however, late complications were more frequent, the most common being urinary tract infections (4) and parastomal hernias (5). Based on GRA responses, $40 \%$ of patients reported subjective clinical improvement, while the remaining $60 \%$ had either worse or unchanged symptoms.

Conclusions: Our experience with this surgical method demonstrates similar objective outcomes as previous studies [1,2], but also shows that less than half of patients are satisfied with their postoperative symptom response. This finding may reflect the general difficulty in symptom control in a subset of an already challenging patient population and may be confounded by other factors perceived by the patient as bothersome such as postoperative complications. Nevertheless, retubularizing the ileocystoplasty pouch for creation of an ileal conduit offers several therapeutic advantages over creation of a de novo conduit and should be considered as a viable treatment option in this patient population when other less-invasive therapies have failed.

\section{UP-110}

Conservative Management of High Post-void Residual Urine in Asymptomatic Men: Retrospective Analysis of Outcomes

Kalyanaraman, Balaji; Bracken, R. Bruce

University of Cincinnati, Cincinnati, $\mathrm{OH}$, United States

Introduction and Objectives: Elevated post-void residual (PVR) volume (greater than $150 \mathrm{ml}$ ) may occur in symptomatic men with conditions such as benign prostatic hypertrophy, bladder neck contracture, urethral stricture, and neurogenic bladder. Although the prevalence is unknown, there exists a population of men with asymptomatic, elevated PVR. The aim of this study was to examine clinical outcomes of conservative management in a cohort of patients with asymptomatic, elevated PVR.

Methods: This study included men with PVR greater than $150 \mathrm{ml}$ as measured by ultrasound. Imaging was performed to rule out hydronephro- sis. Treatment was instituted if symptoms and/or hydronephrosis were present. Patients without symptoms or hydronephrosis were managed with watchful waiting in the office setting by a single urologist (RBB). Retrospective chart review was done to examine the incidence of febrile urinary tract infections (UTI), worsening renal function, and need for surgical intervention.

Results: Out of 74 patients with asymptomatic, elevated PVR at initial presentation, $17(23 \%)$ become subsequently symptomatic and required surgical intervention. The remaining 57 patients were managed with watchful waiting for median duration of 7 years (1.4-10.3 years). Median PVR in this study population was $287 \mathrm{ml}(151-967 \mathrm{ml})$. Out of this cohort, only two patients $(3.5 \%)$ developed febrile UTI and renal insufficiency. Conclusions: Patients with asymptomatic high PVR can be safely managed conservatively over long-term with watchful waiting.

\section{UP-111}

No Show Rates for Follow-up after Visual Internal Urethrotomy: a Single Surgeon Experience

Patel, Premal'; ; Baverstock, Richard ${ }^{2}$

${ }^{1}$ University of Calgary, Calgary, $\mathrm{AB}$, Canada; ${ }^{2}$ vesia [Alberta bladder centrel, Calgary, AB, Canada

Introduction and Objectives: Visual Internal Urethrotomy (VIU) is the most commonly performed intervention for urethral stricture disease. Although VIU has had excellent outcomes with success rates ranging from $50 \%$ to $85 \%$, longer follow-up studies have shown poorer success rates ranging from $6 \%$ to $28 \%$. Follow-up at 6 months is typically conducted to assess for recurrence as this may indicate the need for a second VIU (1), a decision to move towards urethral reconstruction, or even observation. However, treatment for stricture disease is often hampered by poor patient follow-up. This study aims to assess the percentage of no-show rates post VIU and the potential implicating factors.

Methods: A chart review using the Electronic Medical Record (Wolf), as well as hospital database was assessed. Of 75 patients who had undergone VIU since 2006, 62 met our inclusion criteria. Netcare was also checked to ensure the patient did not go elsewhere for follow-up such as urgent dilation, cystoscopy or VIU.

Results: A total of 76 VIUs were performed on 63 patients. 59 patients were male and 4 were female. Of the male patients, $71 \%$ had bulbar urethral strictures whereas $29 \%$ had urethral strictures. 15 patients required greater than $1 \mathrm{VIU}$ and 5 patients went on to urethral reconstruction. A large percentage of patients (19\%) failed to attend their scheduled followup appointment. The median age of patients failing to attend appointments was 36.5 as opposed to 48 for those who attended.

Conclusions: Patients that received VIUs were half as likely to attend their follow-up appointments than patients attending regular urology cystoscopy practice (19\% vs. $9 \%$ no show, respectively). All patients that did not attend their follow-up appointments were male, and $20 \%$ of those patients required more than one VIU. Improvement of urinary symptoms may influence follow-up rates as they may feel they no longer require medical follow-up. Patient age and contact availability may also be implicated (2).

\section{UP-112}

Genitourinary Tuberculosis or Not Genitourinary Tuberculosis: Case Series and Critical Look at the Challenging Diagnosis of Genitourinary Tuberculosis

Boyle, Shawna $;$; Amman, Justin²; Chin, Joseph ${ }^{3}$; Paulter, Stephen ${ }^{3}$; Denstedt, John D. ${ }^{3}$; Izawa, Jonathan ${ }^{3}$; Razvi, Hassan ${ }^{3}$

${ }^{1}$ University of Western Ontario, London, ON, Canada; ${ }^{2}$ Department of Medical Imaging, The University of Western Ontario, London, ON, Canada; ${ }^{3}$ Division of Urology, Department of Surgery, The University of Western Ontario, London, ON, Canada

Introduction and Objectives: Genitourinary (GU) tuberculosis (TB) can present with a wide variety of symptoms and has no single unifying pathopneumonic presentation. Despite treatment protocols and criteria for high-risk individuals, the number of reported cases of tuberculosis in Canada has been relatively stable over the past 10 years. Approximately $40 \%$ of the cases are in Ontario and 3.5\% of all presentations involve 
the GU system. We aim to review the presentations of GU TB in Canada then consider the diagnostic challenges posed by it.

Methods: We reviewed the medical records and imaging of all cases of GU TB treated in London over the past 8 years. The radiologic and clinical findings for each patient was assessed to reflect the ways in which TB can present in the GU system including a critical look at the diagnostic tools available and their shortcomings.

Results: There were 4 females and 1 male, with an age range at presentation from 45-80 years. Presented are 2 TB kidney, 1 primary bladder who presented with gross hematuria, 1 with ureteric stricture disease and 1 with a testicular mass. All of these cases were confirmed with acid fast bacilli stains on tissue or urine specimens. None of the patients had a personal history of known TB. However, all had radiologic findings consistent with TB on retrospective review. Four of the 5 patients were not born in Canada but all had negative screening chest $\mathrm{x}$-rays and TB skin testing at immigration was self reported as negative.

Conclusions: It is conceivable that these patients did not acquire TB in Canada and tests used for TB screening at immigration may be inaccurate. A comparison of the sensitivity and specificity of urine studies, tissue studies, radiologic findings and the use of more accurate testing such as urine PCR studies, showed that GU TB remains a diagnostic dilemma. Although a rare diagnosis, it is not one which should be forgotten in our differential, as it is neither historic nor declining in its rates of presentation.

\section{UP-113}

\section{Common Urological Presentations of the Penitentiary Population} in Kingston: a Chart Review

Lee, Taehyoung; Leveridge, Michael; Touma, Naji

Queen's University, Kingston, ON, Canada

Introduction and Objectives: Kingston, Ontario provides for approximately $20 \%$ of incarcerated offenders under custody of Correctional Service of Canada (CSC). Therefore, health care institutions in Kingston play a crucial role in providing health care to this unique population. However, there has been a paucity of reports on the penitentiary population with urologic complaints. In this study, we investigated urologic presentations of inmate patients at Kingston General Hospital (KGH).

Methods: A retrospective chart review of 188 consecutive incarcerated patients was performed, accounting for all KGH Urology visits by inmates in the past 5 years. All patients were under custody of CSC at the time of their first visit. Patient charts were assessed to determine age, gender, presenting signs and symptoms, diagnoses, and treatments provided. The demographic and diagnostic distributions were analyzed; the pattern of presenting signs and symptoms was also investigated.

Results: All 188 inmates were males between the age of 25 and 87 at the time of the study. The most common age group to present with urologic complaints was between the ages of 50 and 59. The most prevalent signs and symptoms included lower urinary tract symptoms $(27.7 \%)$ and hematuria $(19.1 \%)$, followed by renal colic $(5.3 \%)$ and symptoms of neurogenic bladder (2.7\%). Out of 188 patients, $117(62.2 \%)$ received a definitive diagnosis. Common diagnoses included nephrolithiasis/ureterolithiasis $(11.2 \%)$, benign prostatic hyperplasia $(8.0 \%)$, and hydrocele $(6.9 \%)$, followed by epididymo-orchitis $(4.3 \%)$, prostate cancer $(4.3 \%)$, hydronephrosis $(3.2 \%)$, phimosis/paraphimosis $(2.7 \%)$ and urethral stricture $(2.7 \%)$.

Conclusions: In this study, we aimed to outline the nature of urologic presentations in the Canadian penitentiary population. As a subsequent step, we will compare the presenting urological problems of the inmate population to the general population, and identify potential barriers for care or access.

\section{UP-114}

Targeted Robotic-assisted Microsurgical Denervation of the Spermatic Cord for Chronic Orchialgia

Gudeloglu, Ahmet; Wharton, Jessica; Priola, Karen; Parekattil, Sijo

University of Florida and Winter Haven Hospital, Winter Haven, FL, United States

Introduction and Objectives: Previous groups have shown microsurgical denervation of the spermatic cord (MDSC) as a possible treatment option for chronic orchialgia. Pathology and anatomical studies have identified specific nerve bundles within the spermatic cord that may be responsible for chronic pain in these men. This study presents outcomes for a robotic assisted targeted MDSC approach utilizing a mapped nerve protocol to maximize preservation of vessels and lymphatics.

Methods: This study was a prospective outcomes research study with the primary endpoints of elimination in pain impacting quality of life (assessed utilizing a standardized validated pain assessment tool: PIQ-6 (QualityMetric Inc., Lincoln, RI), operative duration, hydrocele formation and testicular atrophy. Analysis of 348 MDSC cases from October 2008-December 2011 was performed (median follow-up 19 months: 1 to 38). Selection criteria: chronic testicular pain ( $>3$ months), failed all other standard pain management treatments and negative urologic workup. Pain scores and physical exam were performed preoperatively and then postoperatively at $1,3,6,9 \& 12$ months.

Results: $85 \%$ (297/348) of the patients had a significant decrease in their pain (defined as pain having no impact on quality of life questionnaire - score of $\leq 50$ or a greater than $50 \%$ reduction in pain) by 6 months postoperatively. The procedure failed to provide pain relief in 51 patients. Median operative duration was $15 \mathrm{~min}$ (10-150). Complications were: 1 testicular ischemia, 9 hematomas, 2 seromas. There were two testicular arteries and one vasal injury; these were repaired intra-operatively with robotic assisted microsurgical techniques without any further sequel.

Conclusions: Targeted robotic assisted MDSC is feasible and the preliminary results appear promising. Further follow-up and further evaluation is warranted. The four arm robotic approach allows the microsurgeon to maneuver multiple instruments simultaneously.

\section{UP-115}

Should Large Adrenal Myelolipomas $(>6 \mathrm{~cm})$ Be Removed? Case Series and Review of Literature

Michael, Amanda; Elias, Rami; Kapoor, Anil

McMaster University, Hamilton, ON, Canada

Introduction and Objectives: Adrenal myelolipomas (ML) are rare, nonfunctional, benign neoplasms composed of adipose cells and hematopoietic elements. With the widespread use of abdominal imaging, more frequent discovery of these quiescent adrenal masses occurs. While management of small, asymptomatic adrenal $\mathrm{ML}$ is well described, the management of large $(>6 \mathrm{~cm})$, incidental adrenal $\mathrm{ML}$ remains controversial. Most advocate surgical resection given the concern of life-threatening, spontaneous, retroperitoneal hemorrhage. The literature was reviewed to evaluate current management of incidental adrenal ML larger than $6 \mathrm{~cm}$. We present four cases of large adrenal ML (7 to $10 \mathrm{~cm}$ ) encountered at our institution, laparoscopically excised without complications. Indications for resection included concerns regarding malignant potential. Pathology confirmed the diagnosis of adrenal ML with no concomitant malignancy in each case.

Methods: A comprehensive review of the literature was performed by two independent searchers, using both PubMed and MEDLINE databases with the key words adrenal and myelolipoma.

Results: More than 300 abstracts were reviewed, 117 of which were assessed in detail. Salient clinical and pathologic characteristics, in addition to criteria for definitive radiological diagnosis and the role of fine needle aspiration biopsy for diagnostic confirmation are described.

Conclusions: Large, asymptomatic adrenal ML diagnosed definitively with imaging can be managed conservatively. No malignant potential for adrenal ML has been recorded. Similarly, our clinical experience with laparoscopic adrenalectomy for large adrenal ML confirms no evidence of malignancy for masses greater than $6 \mathrm{~cm}$. When clinical features and imaging are indicative of adrenal $\mathrm{ML}$, a surgical approach is not warranted merely on account of size. The literature indicates an absence of a correlation between ML size and risk of hemorrhage. No mortalities have been reported secondary to ruptured adrenal ML.

\section{UP-116-WITHDRAWN}

